Florida International University FIU Digital Commons

$5-25-2012$

\title{
Functionalized Carbon Micro/Nanostructures for Biomolecular Detection
}

Varun Penmatsa

vpenm001@fiu.edu

DOI: $10.25148 /$ etd.FI12112702

Follow this and additional works at: https://digitalcommons.fiu.edu/etd

\section{Recommended Citation}

Penmatsa, Varun, "Functionalized Carbon Micro/Nanostructures for Biomolecular Detection" (2012). FIU Electronic Theses and Dissertations. 739.

https://digitalcommons.fiu.edu/etd/739 


\section{FLORIDA INTERNATIONAL UNIVERSITY}

Miami, Florida

\section{FUNCTIONALIZED CARBON MICRO/NANOSTRUCTURES FOR BIOMOLECULAR DETECTION}

A dissertation submitted in partial fulfillment of the requirements for the degree of DOCTOR OF PHILOSOPHY

in

MATERIALS SCIENCE AND ENGINEERING

by

Varun Penmatsa

2012 
To: Dean Amir Mirmiran

College of Engineering and Computing

This dissertation, written by Varun Penmatsa, and entitled Functionalized Carbon Micro/Nanostructures for Biomolecular Detection, having been approved in respect to style and intellectual content, is referred to you for judgment.

We have read this dissertation and recommend that it be approved.

$\begin{array}{r}\hline \text { W. Kinzy Jones } \\ \hline \text { Norman D.H. Munroe } \\ \hline \text { Chenzhong Li } \\ \hline \text { Chunlei Wang, Major Professor }\end{array}$

Date of Defense: May 25, 2012

The Dissertation of Varun Penmatsa is approved.

Dean Amir Mirmiran College of Engineering and Computing

Dean Lakshmi N. Reddi University Graduate School

Florida International University, 2012 
(C) Copyright 2012 by Varun Penmatsa

All rights reserved. 


\section{DEDICATION}

I dedicate this dissertation to my loving parents, Chiranjeevi Raju and Subhashini Penmatsa for their endless love, guidance and support. This dissertation is also dedicated to my late grandfather, Mr. Subba Raju, who would be extremely proud of this accomplishment. 


\section{ACKNOWLEDGMENTS}

This dissertation is made possible with the help of so many people in so many ways. I wish to sincerely thank my advisor Dr. Chunlei Wang, for her cardinal role in my education and personal development through her guidance, support and encouragement. She has always given me the freedom to pursue independent work and explore different exciting topics. The last five years I spent in her lab for my graduate studies have been an amazing journey, and along the way I had the opportunity to meet some amazing individuals. Her meticulous review and constructive criticism of my research work has led to many "why didn't I think of that" moments and helped shape me into a better researcher.

I wish to extend my sincere thanks to my dissertation committee members, Dr. Kinzy Jones, Dr. Norman Munroe and Dr. Chenzhong Li for their advice and continous support. I owe many thanks to Dr. Jones for his technical guidance and valuable suggestions going back to- when he also served on my Master's thesis committee. I wish to acknowledge Dr. Munroe and Dr. Chenzhong Li for always being accessible for research discussions and support when needed. I also would like to thank other professors in my department for providing me with tremendous graduate education.

This work would not have been possible without the support from Dr. Hiroshi Kawarada's group at Waseda University, Japan. I wish to extend my sincere gratitude to Dr. Kawarada for giving me the opportunity to visit his lab and the help he provided during my stay in Japan. Special thanks to Ruslinda. A. Rahim and Yuchiro Ishiyama for their experimental assistance and helping me to blend in with the Japanese culture. The Nomihoudai and Tabehoudai with his research group are times that I will always cherish. 
With five years in Dr. Wang's group, I have a lot of colleagues and friends to thank. I wish to acknowledge all my past and present lab mates, Dr. Jung-hoon Yang, Dr. Yan Yu, Dr. Wei Chen, Dr. Xifei Li, Taekwon Kim, Majid Beidaghi, Abirami Dhanabalan, Yin Song, Richa Agarwal, Yong Hao, Yamini Parikh, Gregg Burrow, Sheidyn Ng and Kevin Bechtold for their friendship and help in my research.

I would like to acknowledge the excellent facilities provided at Advanced Materials Engineering Research Institute (AMERI), The Center for the Study of Matter at Extreme Conditions (CeSMEC) at FIU and Nanotechnology Research Centre (NTRC), Waseda University, Japan. I thank Mr. Neal Ricks for his patience while teaching me to work with different equipment and technical assistance provided during the course of my work. It has also been an honor to work closely with talented engineers at AMERI, CeSMEC and NTRC helping me to finish this work in a timely fashion.

My experience at FIU beyond our research group has been equally amazing with a long list of great friends and colleagues. I would like to thank all these amazing friends I made during the last few years for their support and encouragement. My thanks to Dr. Arvind Agarwal and the Material Advantage chapter for giving me the opportunity to be part of the amazing executive team. The time in this chapter has helped broaden my leadership foundation.

I also extend my sincere thanks to my pre-FIU mentor Mr. J. Kundu for inspiring me to dream big. Finally, to my mom and dad, grandparents, sister and brother-in-law for always being there for me. These acknowledgements would not be complete without mentioning my dear niece Jagati and nephew Abhijay for always showering their energetic and boundless love on me. 


\section{ABSTRACT OF THE DISSERTATION \\ FUNCTIONALIZED CARBON MICRO/NANOSTRUCTURES FOR BIMOLECULAR \\ DETECTION \\ by \\ Varun Penmatsa}

Florida International University, 2012

Miami, Florida

Professor Chunlei Wang, Major Professor

Advancements in the micro-and nano-scale fabrication techniques have opened up new avenues for the development of portable, scalable and easier-to-use biosensors. Over the last few years, electrodes made of carbon have been widely used as sensing units in biosensors due to their attractive physiochemical properties. The aim of this research is to investigate different strategies to develop functionalized high surface carbon micro/nanostructures for electrochemical and biosensing devices.

High aspect ratio three-dimensional carbon microarrays were fabricated via carbon microelectromechanical systems (C-MEMS) technique, which is based on pyrolyzing pre-patterned organic photoresist polymers. To further increase the surface area of the carbon microstructures, surface porosity was introduced by two strategies, i.e. (i) using F127 as porogen and (ii) oxygen reactive ion etch (RIE) treatment. Electrochemical characterization showed that porous carbon thin film electrodes prepared by using F127 as porogen had an effective surface area $\left(A_{\text {eff }} 185 \%\right)$ compared to the conventional carbon electrode. 
To achieve enhanced electrochemical sensitivity for C-MEMS based functional devices, graphene was conformally coated onto high aspect ratio three-dimensional (3D) carbon micropillar arrays using electrostatic spray deposition (ESD) technique. The amperometric response of graphene/carbon micropillar electrode arrays exhibited higher electrochemical activity, improved charge transfer and a linear response towards $\mathrm{H}_{2} \mathrm{O}_{2}$ detection between $250 \mu \mathrm{M}$ to $5.5 \mathrm{mM}$.

Furthermore, carbon structures with dimensions from 50 nano-to micrometer level have been fabricated by pyrolyzing photo-nanoimprint lithography patterned organic resist polymer. Microstructure, elemental composition and resistivity characterization of the carbon nanostructures produced by this process were very similar to conventional photoresist derived carbon. Surface functionalization of the carbon nanostructures was performed using direct amination technique.

Considering the need for requisite functional groups to covalently attach bioreceptors on the carbon surface for biomolecule detection, different oxidation techniques were compared to study the types of carbon-oxygen groups formed on the surface and their percentages with respect to different oxidation pretreatment times.

Finally, a label-free detection strategy using signaling aptamer/protein binding complex for platelet-derived growth factor oncoprotein detection on functionalized threedimensional carbon microarrays platform was demonstrated. The sensor showed near linear relationship between the relative fluorescence difference and protein concentration even in the sub-nanomolar range with an excellent detection limit of 5 pmol. 


\section{TABLE OF CONTENTS}

CHAPTER

PAGE

1. INTRODUCTION

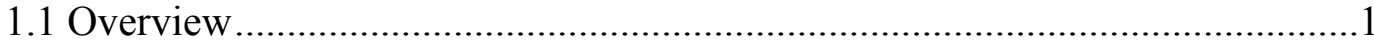

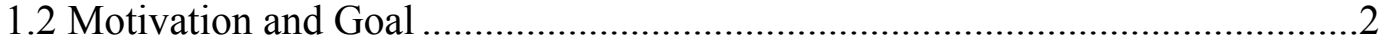

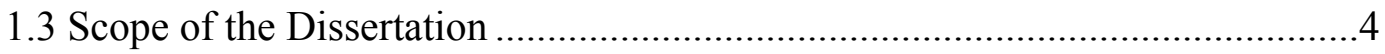

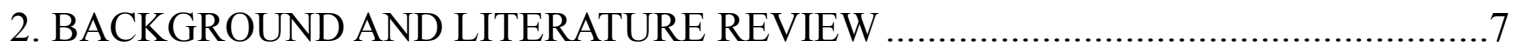

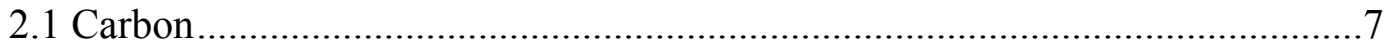

2.7.1 Brief History of Carbon ...................................................................

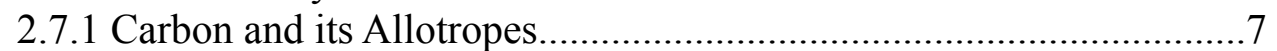

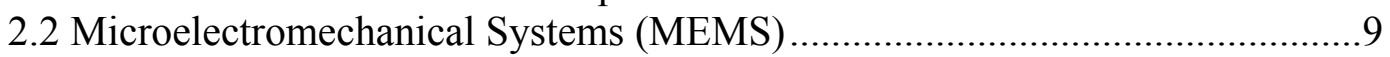

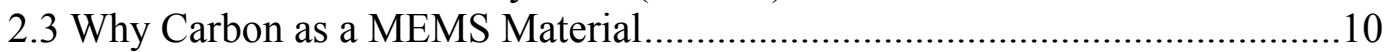

2.4 Carbon Microelectromechanical Systems...................................................12

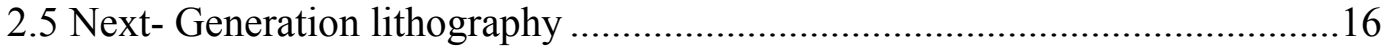

2.5.1 Nanoimprint Lithography ...........................................................16

2.6 C-MEMS based Biosensing .......................................................................18

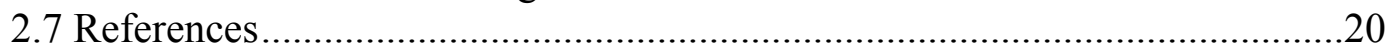

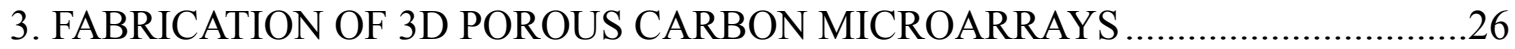

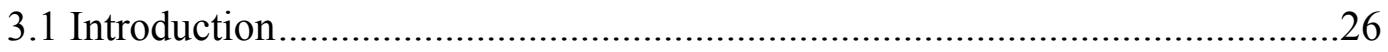

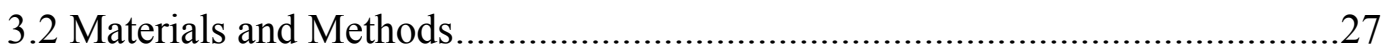

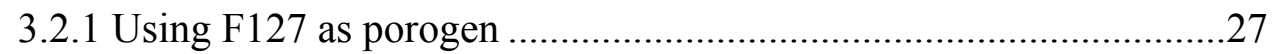

3.2.1.1 Precursor Preparation......................................................2

3.2.1.2 Modified C-MEMS Process............................................28

3.2.1.3 Two-Dimensional Carbon Electrodes for Electrochemical

Characterization .............................................................29

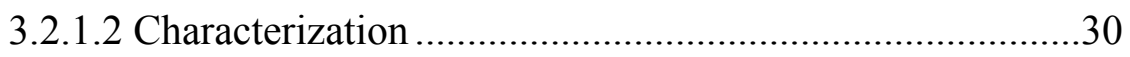

3.2.2 Using Oxygen RIE Treatment ........................................................31

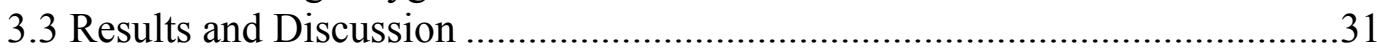

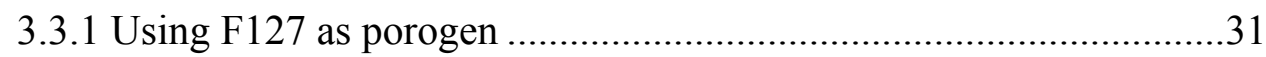

3.3.1.1 Thermal Characterization...................................................31

3.3.1.2 Morphology Characterization ............................................34

3.3.1.3 Electrochemical Characterization ........................................36

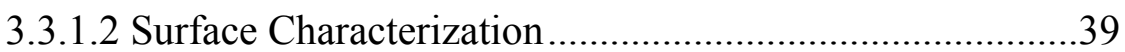

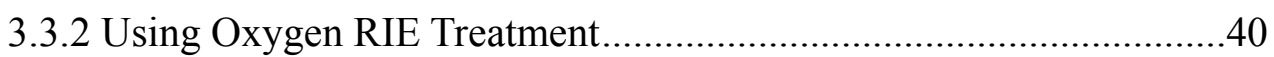

3.3.2.1 Surface Morphology Characterization...............................40

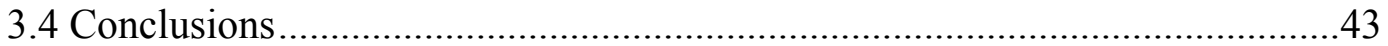

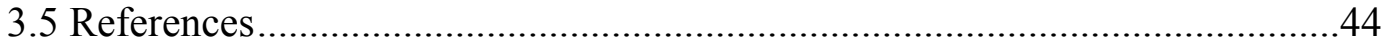

4. THREE-DIMENSIONAL GRAPHENE COATED CARBON MICROPILLAR ARRAYS FOR ENHANCED ELECTROCHEMICAL SENSING ……...........................46

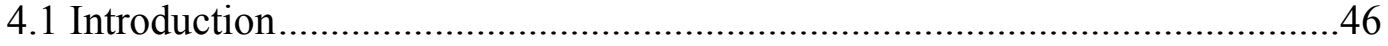




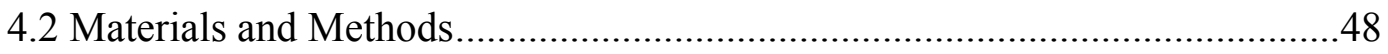

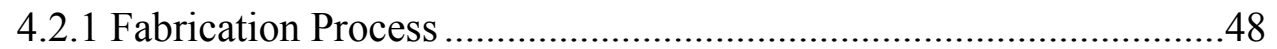

4.2.2 Electrostatic Spray Deposition (ESD) Process …….........................49

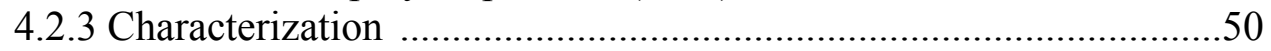

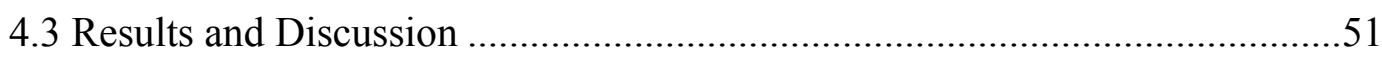

4.3.1 Fabrication and Characterization …………………........................51

4.3.2 Effect of ESD Processing Conditions ................................................54

4.3.3 Hydrogen Peroxide Detection ......................................................59

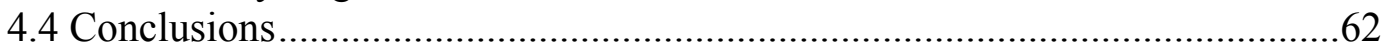

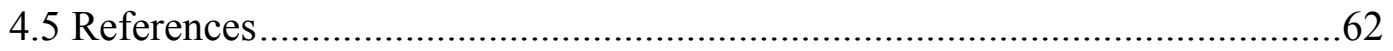

5. WAFER-LEVEL FABRICATION OF FUNCTIONALIZED CARBON NANOSTRUCTURES WITH CONTROLLABLE SIZE, SHAPE AND POSITION .....67

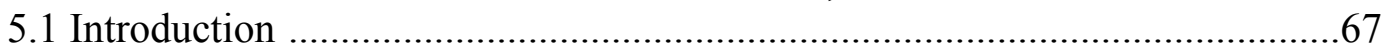

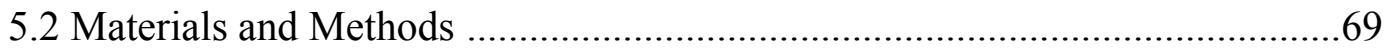

5.2.1 Fabrication Process ............................................................................

5.2.2 Surface Functionalization .............................................................

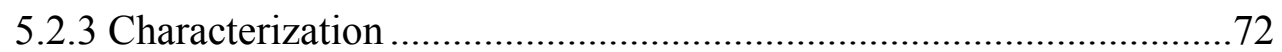

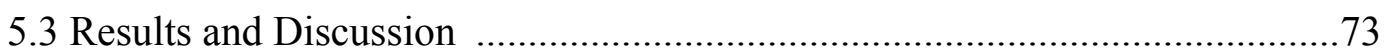

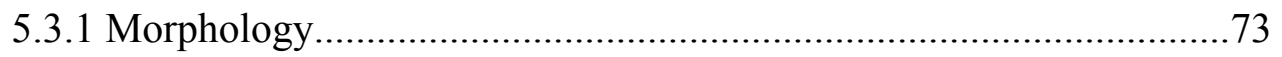

5.3.2 Thermal Analysis .........................................................................

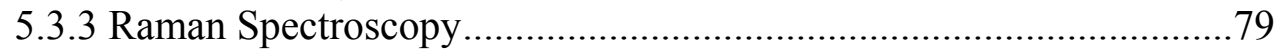

5.3.4 X-Ray Photoelectron Spectroscopy …………………………..........8

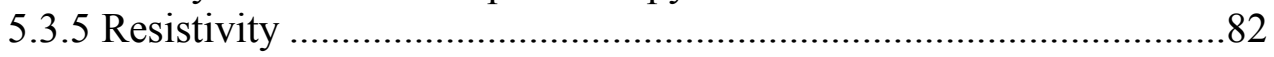

5.3.6 Surface Functionalization ...........................................................8

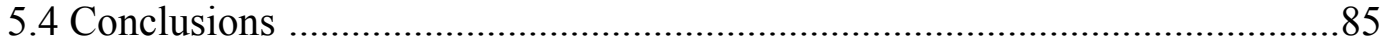

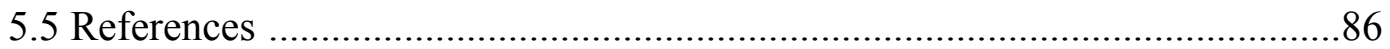

\section{COMPARISON OF DIFFERENT OXIDATION TREATMENTS ON C-MEMS}

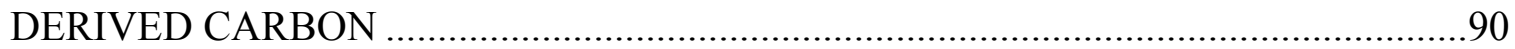

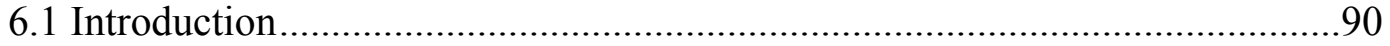

6.2 Materials and Methods.................................................................................91

6.2.1 Vacuum Ultraviolet (VUV) Pretreatment ......................................91

6.2.2 Electrochemical Activation Pretreatment ........................................93

6.2.3 UV/Ozone Pretreatment.................................................................94

6.2.4 Oxygen RIE Pretreatment ............................................................95

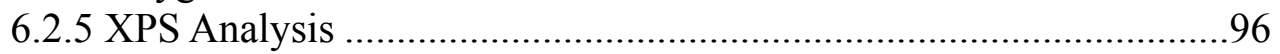

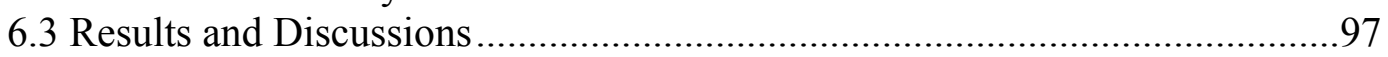

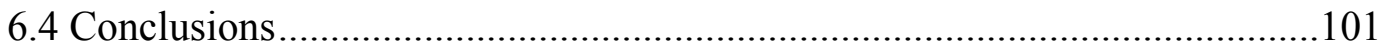

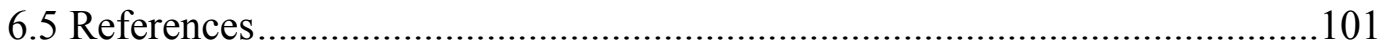

7. DETECTION OF PLATELET-DRIVED GROWTH FACTOR (PDGF) USING

SIGNALING APTAMER/ PROTEIN BINDING COMPLEX …………………............104

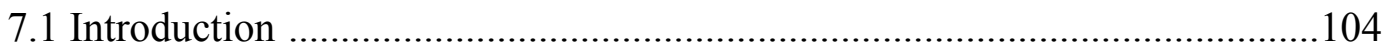

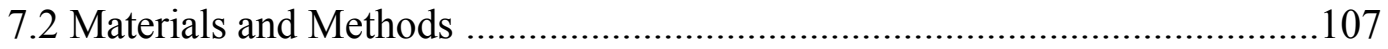


7.2.1 Fabrication of 3D Carbon Micropillar Arrays …………..................107

7.2.2 Surface Functionalization ............................................................108

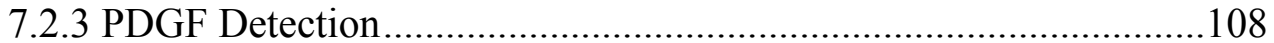

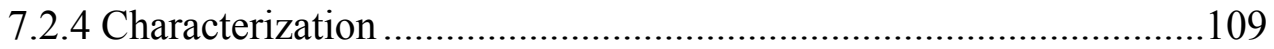

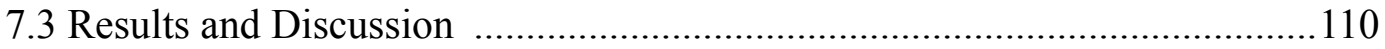

7.3.1 Characterization ........................................................................110

7.3.2 Surface Functionalization .............................................................111

7.3.3 Signaling Aptamer/Protein Binding Complex Mechanism...............112

7.3.4 Sensitivity and Selectivity of the Sensor .........................................114

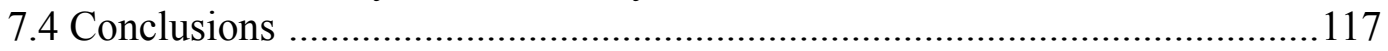

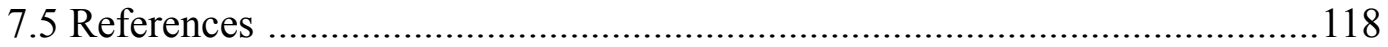

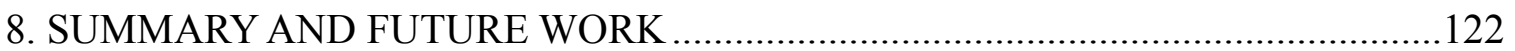

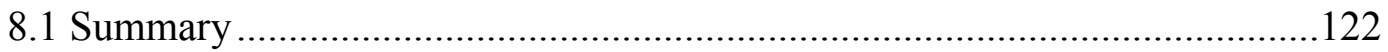

8.2 Future Scope of this Work ....................................................................124

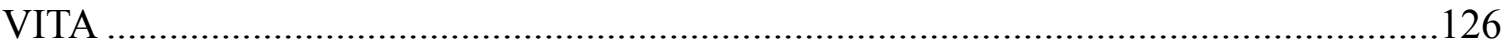




\section{LIST OF FIGURES}

FIGURE

PAGE

Figure 1.1 SEM images of assorted microstructures fabricated using C-MEMS process ..3

Figure 1.2 Flowchart of the Research Plan 4

Figure 2.1 Some carbon allotropes: a) Diamond, b) Graphite indicating individual graphene layers, c) Lonsdaleite, d) C60 (buckyball), e) C540, f) C70, g) Amorphous carbon and h) Single walled carbon nanotube ..8

Figure 2.2 Carbon products obtained from different processes.

Figure 2.3 Schematic showing the C-MEMS process using a negative tone resist (SU-8) as the precursor

Figure 3.1 Schematic of the fabrication process: (a) spin coating the photoresist + F127 precursor to obtain a uniform film on the substrate, (b) patterning the photoresist precursor using UV light, (c) the resultant patterned array of three dimensional microstructures after developing using suitable developer, (d) soaking three-dimensional microstructures in water bath at $80^{\circ} \mathrm{C}$ to induce gelation of $\mathrm{F} 127$, (e) subsequent vacuum treatment to eliminate bubbles formed, and (f) carbonizing the polymer microstructures under forming gas atmosphere at high temperature (a) Schematic of a typical process flow for fabricating C-MEMS electrodes. .29

Figure 3.2 Thermal behavior of pure SU-8, F127 mixed with SU-8 and pure F127 investigated from $30{ }^{\circ} \mathrm{C}$ to $500{ }^{\circ} \mathrm{C}$ by DSC

Figure 3.3 Thermal behavior of pure SU-8, F127 mixed with SU-8 and pure F127 investigated from $30{ }^{\circ} \mathrm{C}$ to $900{ }^{\circ} \mathrm{C}$ by TGA.

Figure 3.4 SEM images of three-dimensional micropillars derived from F127 mixed SU-8 (tilted at $60^{\circ}$ ) precursor after water annealing and vacuum treatment: (a) micropillar arrays with a single micropillar in the inset and (b) high magnification SEM image of the surface showing porous morphology .35

Figure 3.5 SEM images of three-dimensional microstructure derived fromF127 mixed SU-8 after carbonization: (a) porous carbon micropillar arrays and (b) high magnification SEM images of the porous carbon film. .36

Figure 3.6 (a) Cyclic voltammograms of carbon electrodes derived from SU-8 mixed with (a) no F127, 2.5\% F127, 5\% F127, 7.5\% F127 and 10\% F127 in a $10 \mathrm{mM}$ $\mathrm{K}_{3} \mathrm{Fe}(\mathrm{CN})_{6}+3 \mathrm{M} \mathrm{KCl}$ solution. The working electrode setup is shown in the inset.........37 
Figure 3.7 Schematic showing the ratio of increase in the surface area according to the different F127 (\% w/w) concentrations 39

Figure 3.8 AFM image of thin film derived from 10\% F127 mixed photoresist (a) before and (b) after carbonization 40

Figure 3.9 Typical SEM images of carbon micropillars etched at pressure $400 \mathrm{mTorr}$, power $100 \mathrm{~W}$, gas flow $100 \mathrm{sccm}$ for (a) $20 \mathrm{sec}$, (b) $40 \mathrm{sec}$, (c) $60 \mathrm{sec}$, and (d) $80 \mathrm{sec} . . . .41$

Figure 3.10 High magnification SEM images of carbon surface treated for (a) $60 \mathrm{sec}$, (b) 140 sec @100 mTorr pressure, 150 W power, 10 sccm gas flow .42

Figure 3.11 Typical SEM images of carbon micropillars etched at (a) pressure$400 \mathrm{mTorr}$, power- $150 \mathrm{~W}$, gas flow $10 \mathrm{sccm}$, (b) pressure $100 \mathrm{mTorr}$, power $100 \mathrm{~W}$, gas flow $8 \mathrm{sccm}$, (c) pressure-100 mTorr, power-150 W, gas flow $8 \mathrm{sccm}$ for $60 \mathrm{sec}$. .43

Figure 4.1 Photograph of the actual ESD setup in our lab. .50

Figure 4.2 Schematic showing a-d) the fabrication of 3D carbon micropillars, and e) deposition of graphene on carbon micropillars using ESD technique. .52

Figure 4.3 a) Typical SEM image of pristine graphene deposited on silicon oxide, b) HRTEM image of graphene nanosheets, and c) Raman spectra (G and D peaks) of asdeposited graphene nanosheets. .53

Figure 4.4 Typical SEM images of graphene film deposited on carbon at a) $200{ }^{\circ} \mathrm{C}, \mathrm{b}$ ) $300{ }^{\circ} \mathrm{C}$, c) $400{ }^{\circ} \mathrm{C}$ for $30 \mathrm{~min}, 3 \mathrm{~cm} \mathrm{NSD}$;) $3 \mathrm{~cm}$, e) $5 \mathrm{~cm}$, f) $8 \mathrm{~cm} \mathrm{NSD}$ for $60 \mathrm{~min}, 200$ ${ }^{\circ} \mathrm{C}$. Scale bar is $20 \mu \mathrm{M}$.

Figure 4.5 Typical SEM images of graphene deposited carbon micropillars at conditions a) $200{ }^{\circ} \mathrm{C}$ for $60 \mathrm{~min}$; b) $300{ }^{\circ} \mathrm{C}$ for $30 \mathrm{~min}$; Inset: graphene coated carbon micropillar c) Schematic showing the proposed methodology explaining the formation of porous film structure. .57

Figure 4.6 Broadscan XPS spectra of graphene before and after deposition; Inset: Deconvoluted high resolution carbon XPS spectrum. .59

Figure 4.7 Typical cyclic voltammetry curves comparing the amperometric response of bare and graphene-coated carbon micropillar electrode arrays. .60

Figure 4.8 Amperometric response of graphene/carbon micropillar electrode array for successive additions of $250 \mu \mathrm{M}$ hydrogen peroxide into ( $\mathrm{pH}$ 7.2) phosphate buffer; Inset: Calibration curve of graphene/ carbon micropillar electrode array with different concentrations of $\mathrm{H}_{2} \mathrm{O}_{2}$. Operating potential: $+0.4 \mathrm{~V}$ .61 
Figure 5.1 Schematic representation of the direct-amination functionalization process ..71

Figure 5.2 Schematic showing the fabrication process. a) Spin coat thin layers of nanoimprint resist and the photoresist on the substrate, b) Imprint a pre-designed glass mold to transfer the pattern onto the polymers and cure it with UV exposure, c) Retreat the glass mold to notice the pattern, d) Oxygen RIE treatment to etch residual nanoimprint resist, e) Etch the residual photoresist top layer by $\mathrm{CF}_{4}+$ oxygen reactive ion etch, and f) Carbonizing the polymer microstructures under forming gas atmosphere at high temperature .74

Figure 5.3 SEM images of carbon nanostructures. a) Carbon nanostructures with different designs patterns and feature sizes, b) $50 \mathrm{~nm}$ linear nanogratings, c) Comb design with $500 \mathrm{~nm}$ pattern critical dimensions, d) Mesh pattern having $180 \mathrm{~nm}$ critical dimension features e) Chequered pattern with smallest feature size of $70 \mathrm{~nm}$ maintaining its lateral fidelity after pyrolysis. 75

Figure 5.4 AFM images of the P-NIL patterned AR-UL-01 nanostructures a) Before and b) After pyrolysis

Figure 5.5 Thermal behavior of AR-UL-01 and SU-8 investigated from $50{ }^{\circ} \mathrm{C}$ to $900{ }^{\circ} \mathrm{C}$ by a) DSC and b) TGA graphs under inert atmosphere. In the curves, (1) Indicates the region where there is significant loss of solvents, (2) Decomposition of $\mathrm{C}_{3}$ and other byproducts occur (3) Temperature region where solid carbon forms; The peaks at (I) Indicate the decomposition of photoactive compound and (II) The exothermic peak due to the thermal crosslinking of the polymer. .79

Figure 5.6 Raman spectra showing the comparison of thin carbon films prepared from AR-UL-01 and SU-8 80

Figure 5.7 XPS spectra of AR-UL-01 before and after pyrolysis. a) Broad scan spectra, b) Carbon, c) Nitrogen d) Oxygen narrow peaks respectively

Figure 5.8 Schematic showing the test bridge structure used for measuring the sheet resistance. .83

Figure 5.9 Summary of broadscan XPS spectra of carbon surface functionalized by direct amination technique at different treatment times .84

Figure 5.10 Comparison of high resolution XPS spectra of carbon surface before and after 6 hrs direct amination treatment.

Figure 6.1 (a) Schematic showing the VUV surface treatment system, (b) Actual UER 20-172 VUV system.

Figure 6.2 Photograph of the actual setup used for electrochemical activation .94 
Figure 6.3 The actual $\mathrm{UVy} 253 \mathrm{UV} / \mathrm{O}_{3}$ surface pretreatment system.............................95

Figure 6.4 Photograph of the MARCH CS-1217 RIE system.....................................96

Figure 6.5 Summary of oxygen concentration (at $\%$ ) for different oxidation techniques. .98

Figure 6.6 Relative contributions of $\mathrm{C}-\mathrm{C}\left(\mathrm{sp}^{2}\right.$ and $\left.\mathrm{sp}^{3}\right), \mathrm{C}^{+\mathrm{I}}, \mathrm{C}^{+\mathrm{II}}$ and $\mathrm{C}^{+} \mathrm{III}$ components as derived from the peak fitting procedure for $\mathrm{C}$-MEMS derived carbon surface oxidized by various techniques. 100

Figure 7.1 (a) Typical SEM image of 3D carbon microarrays, (b) Raman spectrum of pyrolyzed photoresist film showing the two prominent bands at 1350 and $1590 \mathrm{~cm}^{-1} \ldots 111$

Figure 7.2 Deconvoluted C1s spectra of pyrolyzed photoresist film after $4 \mathrm{hr}$ direct amination, here dash line shows the original data and solid lines show the fitting curves. Inset shows the widescan XPS spectra of carbon film before and after amination. .112

Figure 7.3 Schematic illustration of the detection of PDGF-BB using signaling aptamer/protein binding complex on 3D carbon microarrays platform; (I) covalent immobilization of PDGF-binding aptamer on partially aminated carbon surface, (II) intercalating the probe aptamer with TOTO fluorescent dye, (III) binding PDGF-BB to the aptamer-intercalating dye complex, (IV) regenerating the sensor by sodium dodecyl sulfate (SDS) treatment to remove PDGF and release the intercalating dye.

Figure 7.4 Relative fluorescence difference response of the sensor to different concentrations of PDGF from $0.005 \mathrm{nM}$ to $100 \mathrm{nM}$. The concentrations of the aptamer and intercalating dye were $20 \mu \mathrm{M}$ and $10 \mu \mathrm{M}$, respectively .115

Figure 7.5 Comparison of relative fluorescence difference of different proteins towards PDGF binding aptamer; The concentration of the different molecules (PDGF-BB, PDGFAB, PDGF-AA, BSA, ATP and calmodulin) was 100nM and concentrations of PDGFbinding aptamer and intercalating dye were $20 \mu \mathrm{M}$ and $10 \mu \mathrm{M}$, respectively. 


\section{CHAPTER 1}

\section{INTRODUCTION}

\subsection{Overview}

In the last few decades, miniature bio-detection systems have been extensively studied for their potential to replace costly time-consuming diagnostic tools [1]. The early detection and diagnosis of pathogenic and physiologically relevant molecules in the body can significantly reduce the cost of patient care associated with advanced stages of many health disorders [2]. The current laboratory diagnostic methods for identifying these molecules face several potential limitations such as costly detection processes, large sample volumes, need for trained personnel and slow response times [2].

Current biosensors research is fast-moving towards addressing the critical need for developing scalable, low cost, highly sensitive and selective devices that can operate in real-time and diverse physical environments. However, for the successful development of such advanced biosensors, two important factors needs to be addressed from research point of view, i.e. (i) seek novel materials for sensing elements that have well defined and highly stable interfaces when interacting with biomolecules; (ii) develop new miniature designs and manufacturing strategies. The sensing element is considered an integral part of the biosensor design due to its close contact with biomolecules such as enzyme, organelles, whole cells, to detect specific analyte [3]. Miniaturization of detection system enables the integration of more sensing elements units on the allotted substrate area of a sensor system. This enables a far greater number of detection sites between the measurement system and the analyte, thus providing the possibility for realizing high throughput, parallel detection system. In addition, as the electrode dimensions is 
reduced, the radial diffusion becomes more dominant resulting in faster mass transport [4]. The high rate of mass transport at small electrodes enables measurements of kinetics by steady-state experiments rather than by transient techniques.

\subsection{Motivation and Goal}

Over the last few years, electrodes made of carbon has been widely investigated as sensing units in biosensors due to their attractive properties such as low cost, ability to be micro/nano-patterned with high fidelity, easy surface functionalization, wide potential window, and biocompatibility. Compared to other typically used materials such as silicon, glass and gold, carbon does not exhibit significant degradation of the interfaces after repeated use [5]. However, to integrate carbon into miniaturized MEMS based biosensing devices, the biggest challenge is the need to develop fabrication strategies that are compatible with microelectronics processing and at the same time provide the requisite sensitivity and stability when exposed to biological environments.

Carbon microelectromechanical systems, or C-MEMS describes a manufacturing technique in which carbon microstructures are fabricated by heat treatment of patterned organic polymers at high temperatures under inert environment [6-9]. By changing the processing conditions, complex three-dimensional (3D) carbon structure arrays can be fabricated with different shapes and electrical properties [10]. In addition, the 3D microstructure arrays are considered a very promising platform for integrating functional nanomaterials such as graphene [11], carbon nanotubes [12], and carbon nanospheres [13] to take advantage of potential merits such as very large surface areas and enhanced chemical functionality. In reality, the carbon microstructure arrays serve a dual purpose. First, they render a high surface area platform to increase the binding sites for 
bioreceptors; second, the conductive carbon microstructures and the microfabricated carbon contact pads could be fabricated in the same process for electrochemical sensing[11].

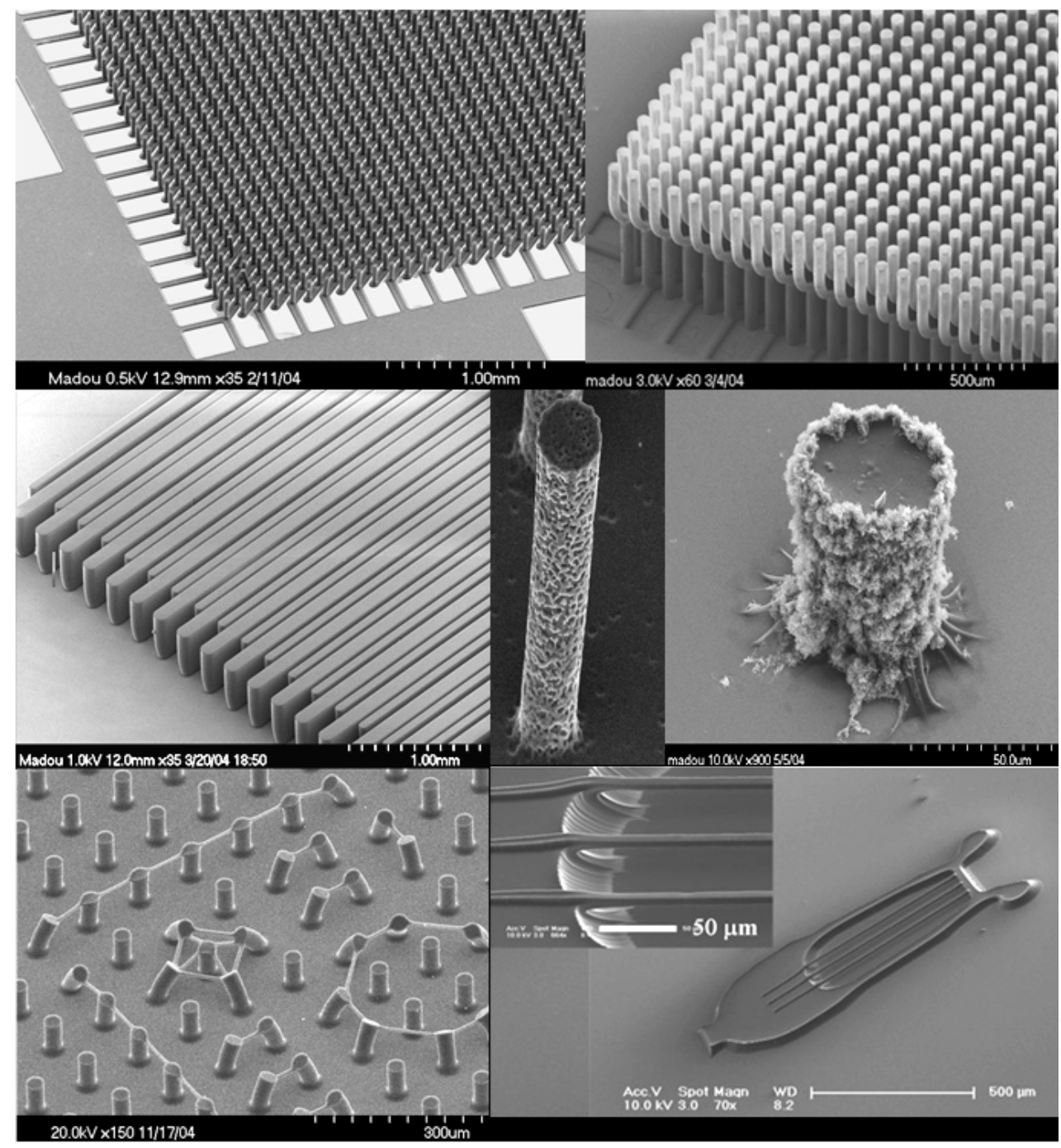

Figure 1.1 SEM images of assorted microstructures fabricated using C-MEMS process. Adapted from Ref [6-10].

The goal of this work is to fabricate, characterize, functionalize and incorporate carbon micro- and nano-structures prepared by C-MEMS/NEMS technique in electrochemical and biodetection devices. To increase the surface area and attain 
enhanced electrochemical functionalities, the surface of the 3D carbon microstructures was engineered to introduce porosity and additionally nanoscale-material, graphene was conformally coated. Furthermore, different functionalization techniques were compared on the pyrolyzed carbon surface to optimize the binding sites covalent attachment of biomolecules. Finally, the functionalized 3D carbon microarrays platform was investigated in electrochemical and potential cancer biomarker detection.

\subsection{Scope of the Dissertation}

The objective of this research is to develop novel strategies to fabricate and test high surface area functionalized carbon micro/nano-structures as electrochemical and biomolecular detection platforms.

Figure 1.2 presents the summary of research carried out in this work

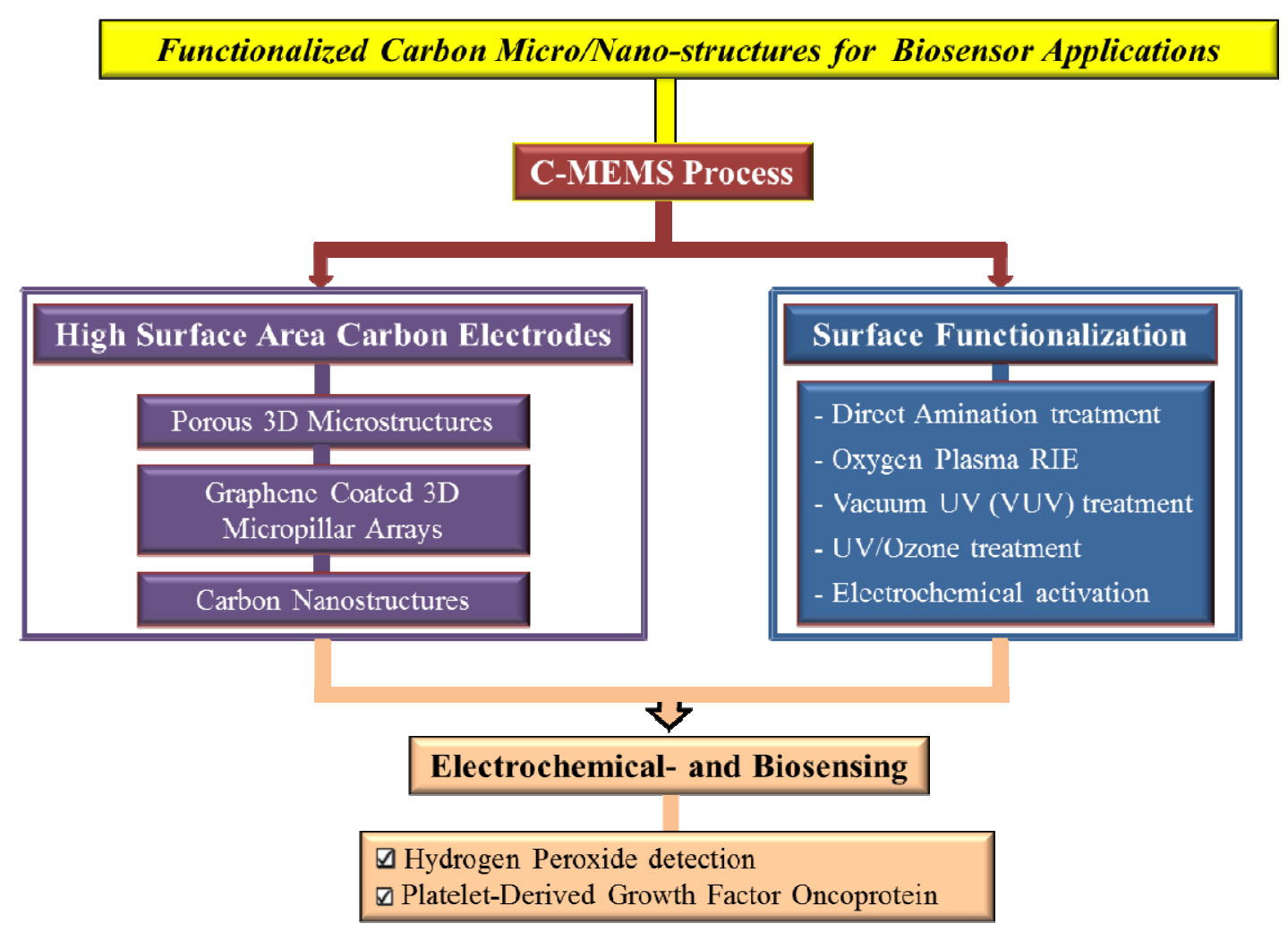

Figure 1.2 Flow chart of the research plan 
This dissertation is divided into 8 chapters.

Chapter 2 presents a thorough background on the allotropes of carbon, need forand significance of carbon microelectrochemical systems (C-MEMS) technique, continues with brief discussion about nanoimprint lithography (NIL) technique and finally the current state-of-art for C-MEMS based biosensing devices is presented. The fabrication and characterization of 3D porous structures using F127 as porogen and oxygen RIE treatment is presented in chapter 3. Chapter 4 discusses a facile approach to integrate graphene nanosheets onto 3D carbon microstructures and testing the electrochemical performance of the sensing platform for hydrogen peroxide detection. Chapter 5 presents the wafer-level fabrication and surface functionalization of carbon nanostructures with controllable size, shape and position using photo-nanoimprint lithography and pyrolysis. The comparison of different oxidation techniques on C-MEMS derived carbon surface was studied to optimize the functional groups responsible for covalent attachment of target binding biomolecules is reported in chapter 6. High sensitive detection of platelet-derived growth factor oncoprotein on functionalized 3D carbon micropillars array platform using signaling aptamer/protein binding complex mechanism is presented in chapter 7. Chapter 8 summarizes the contributions of this dissertation and discusses the future scope of work.

\subsection{References}

[1] T. Vo-Dinh, B. Cullum, "Biosensors and biochips: advances in biological and medical diagnostics. 2000, J. Anal. Chem., 366, 540-551.

[2] S.S. Iqbal, M.W. Mayo, J.G. Bruno, B.V. Bronk, C.A. Batt, J.P. Chambers, $A$ Review of molecular recognition technologies for detection of biological threat agents. 2000, Biosensors and Bioelectronics, 15, 549-578 
[3] F.W. Scheller, F. Schubert, J. Fedrowitz, Frontiers in Biosensorics. 1997, Basel: Birkhauser Verlag, ISBN: 3764354755.

[4] D. W. M. Arrigan, Nanoelectrodes, nanoelectrode arrays and their applications. 2004, Analyst, 129, 1157-1165.

[5] M. F. Phillips, M. R. Lockett, M. J. Rodesch, M. R. Shortreed, F. Cerrina, L. M. Smith, In situ oligonucleotide synthesis on carbon materials: stable substrates for microarray fabrication. 2008, Nucleic Acids Research, 36, e7 (9pp).

[6] C. Wang, L. Taherbadi, M. Madou, A novel method for the fabrication of highaspect ratio C-MEMS structures. 2005, IEEE J. Microelectromechanical Systems, $14,348-358$

[7] S. Ranganathan, R. McCreery, S. Majji, M. Madou, Photoresist-Derived Carbon for Microelectromechanical Systems and Electrochemical Applications. 2000, J. Electrochem. Soc., 147, 277-282

[8] M. Madou, A. Lal, G. Schmidt, X. Song, K. Kinoshita, M. Fendori, A. Zettl, R. White, Carbon micromachining (C-MEMS), 1997, Electrochem. Soc. Proc., 97, 61-69.

[9] C. Wang, R. Zaouk, B. Y. Park, M. Madou, Carbon as a MEMS material: micro and nanofabrication of pyrolysed photoresist, 2008, Int. J. Manufacturing and Management, 13, 360- 375.

[10] J. A. Lee, S. W. Lee, K. C. Lee, S I. Park, S. S. Lee, Fabrication and characterization of freestanding $3 D$ carbon microstructures using multi-exposures and resist, 2008, J. Micromech. Microeng., 18, 035012-035022.

[11] V. Penmatsa, T. Kim, M. Beidaghi, H. Kawarada, Z. Wang, L. Gu, C. Wang, Three-dimensional graphene nanosheets encrusted carbon micropillar arrays for electrochemical sensing, 2012, Nanoscale, DOI:10.1039/C2NR30161JM.

[12] V. Penmatsa, H. Kawarada, C. Wang, 2012., Fabrication of carbon nanostructures using photo-nanoimprint lithography and pyrolysis, 2012, J. Micromech. Microeng. 22, 045024

[13] C. Sharma, S. Patil, S. Saurabh, R. Venkatraghavan, Resorcinol-formaldehyde based carbon nanospheres by electrospraying, 2009, Bull. Mater. Sci., 32, 329246. 


\section{CHAPTER 2}

\section{BACKGROUND AND LITERATURE REVIEW}

\subsection{Carbon}

\subsubsection{Brief History of Carbon}

Carbon is the second most abundant element in the human body by mass (about $18.5 \%$ ) and the fourth most abundant element in the Universe. Etymologically, the English name for carbon was derived from the Latin name for burnt wood, carbo. Dating back to Roman civilization, charcoal derivation was done as we know it today, i.e. by heating wood in a pyramid covered with clay to exclude air [1]. In 1722, Ren'e A. F. de $\mathrm{R}^{\prime}$ eaumur demonstrated that iron could be transformed into steel by the absorption of a certain substance, now known to be carbon [2]. In 1772 the French scientist Antoine de Lavoisier proved that diamond is a crystalline allotrope of carbon by comparing the amount of carbon dioxide/gram released by burning carbon and diamond samples in air [3]. Carl Scheele in 1779, determined that graphite, considered at the time a form of lead, was indeed another carbon allotrope. Lavoisier later listed Carbon as a separate element in his 1789 textbook Trait'e 'El'ementaire de Chimie [4].

\subsubsection{Carbon and its Allotropes}

Carbon forms more compounds than any other element (almost ten million pure organic compounds have been described to date) due to the special nature of the $\mathrm{C}-\mathrm{C}$ bond. Due to the presence of several allotropes of carbon, a large variety of molecular configurations for multi-atomic structures exists. Partial reason for this could be due to the fact that atomic carbon is a very short-lived species that requires to be promptly stabilized [5,6]. Popular allotropes of carbon include graphite, amorphous carbon, 
diamond, glassy carbon, carbon nanotubes [7], buckminster fullerenes [8], lonsdaleite [9], carbyne [10], carbon nanofoams [11], diamond-like carbon [12], carbons derived from the pyrolysis of organic materials known as pyrolyzed carbon [13] and most recently graphene [14]. Some of the typical carbon allotropes are shown schematically in Figure 2.1 .

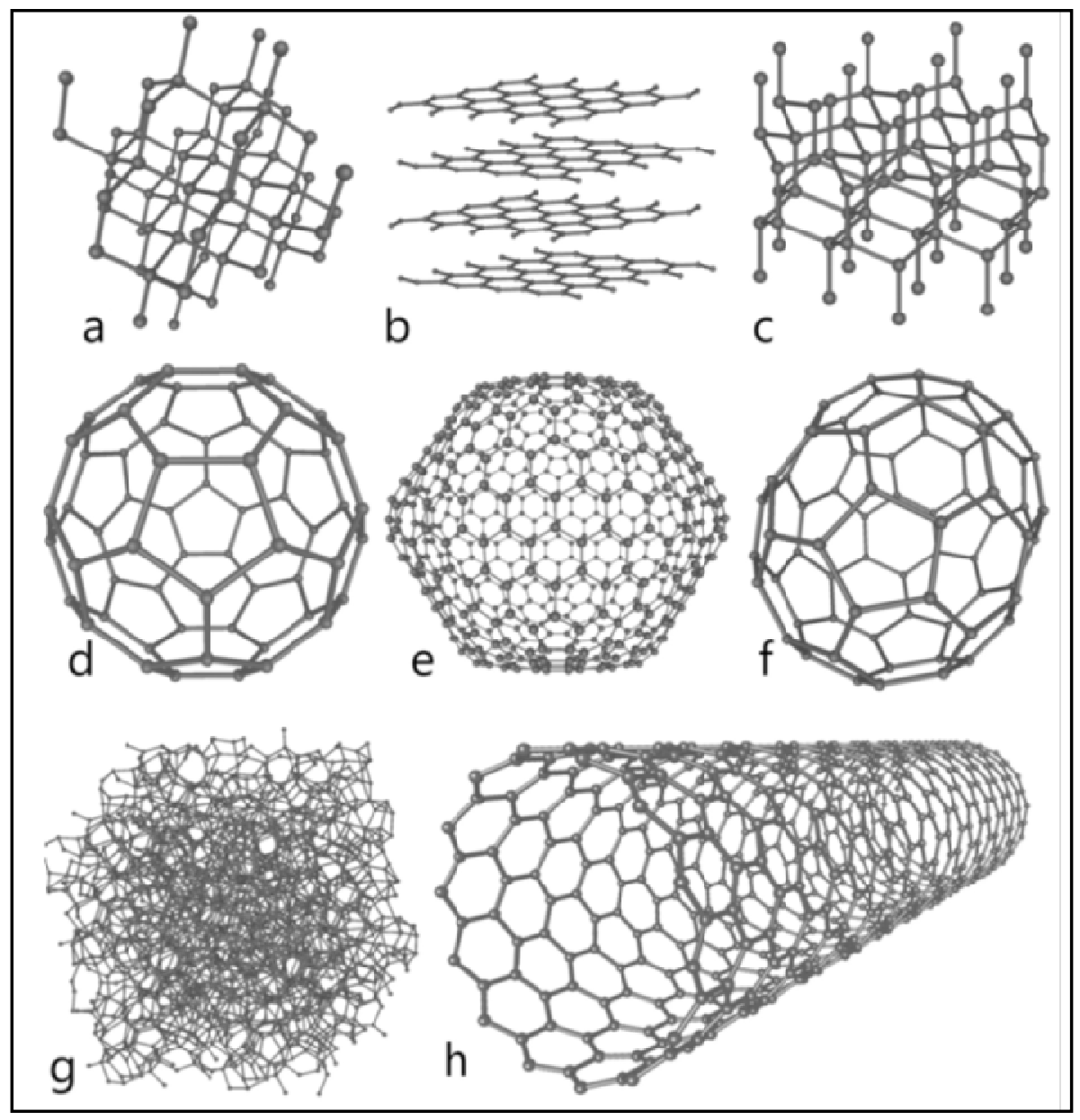

Figure 2.1. Some carbon allotropes: a) Diamond, b) Graphite indicating individual graphene layers, c) Lonsdaleite, d) C60 (buckyball), e) C540, f) C70, g) Amorphous carbon and h) Single walled carbon nanotube. Adapted from Ref [15].

It is noteworthy that the physical, mechanical and electrical properties of carbon vary widely with the allotropic form and their synthesis method. For example, graphite is 
opaque and black while diamond is highly transparent; graphite is soft enough to allow its use as solid lubricant while diamond is amongst the strongest known materials; graphite is a good electrical conductor while undoped diamond is an excellent electrical insulator; some forms of graphite are used in thermal insulation but diamond is the best known naturally occurring thermal conductor. A schematic representing different techniques to derive carbon is shown in Figure 2.2.

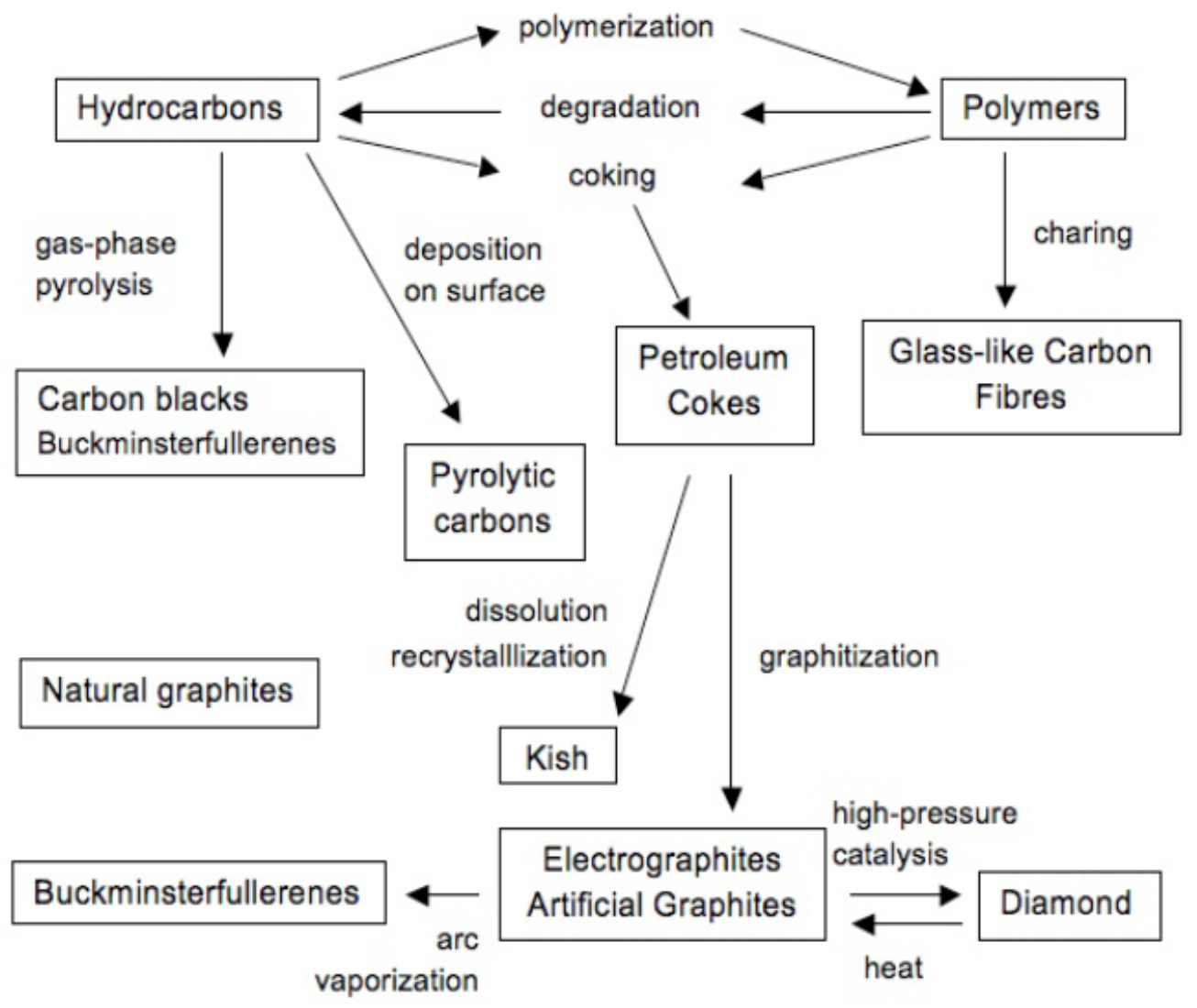

Figure 2.2. Carbon products obtained from different processes. Adapted from Ref [16].

\subsection{Microelectromechanical Systems (MEMS)}

Micromachining or microelectromechanical systems (MEMS) refer to the fabrication of devices with at least some of their dimensions in the micrometer range [17]. In the early years, this discipline was almost exclusively based on thin and thick film processes with 
materials borrowed from Integrated Circuit (IC) fabrications labs. Emphasis was on UV lithography, single-crystal $\mathrm{Si}$, and polycrystalline $\mathrm{Si}$ for mechanical applications such as pressure sensors, accelerometers, and gyros. In the 1990s, as the applications of micromachining broadened, emphasis shifted to a more all-inclusive view of micromanufacturing methods. Unlike IC devices which only involve electrical components (diodes transistors, etc.), MEMS devices cover a broad range of applications from mechanical to biological (BioMEMS). The materials and fabrication techniques used in MEMS are much more varied than those used in IC fabrication (typically uses silicon, oxides and metals patterned using photolithography). In contrast to the IC industry where the devices are meticulously packaged and protected from the environment, MEMS devices, such as glucose or pressure sensors often have surfaces that are directly exposed to the coarse sensing environment. Thus, it is of great interest to investigate a plethora of "exotic" materials that can be "adapted" to make new micro device applications possible. In the case of MEMS applied to medical and biological problems (i.e., BioMEMS), these materials need to have a stable interface when interacting with biological molecules such as aptamers, enzymes, proteins antigens and antibodies.

\subsection{Why Carbon as a MEMS Material?}

Currently, silicon ( $\mathrm{Si}$ ) is the dominant material used in the miniaturization of electrical and electromechanical systems [17]. The most important impetus for the pervasive use of Si in MEMS applications, no doubt, derives from the success of $\mathrm{Si}$ in the Integrated Circuit (IC) industry. The new demands for further miniaturization such as lower power consumption, better heat dissipation, higher speeds, biocompatibility and more environmentally friendly manufacturing processes has fueled our search for 
additional promising materials for MEMS, including $\mathrm{SiC}$ [18], GaAs [19], InP [20], Ge [21], quartz [22], polymeric materials [23] and carbon [24]. In particular, carbon has attracted great interest as a promising new MEMS/Nanoelectromechanical Systems (NEMS) material that can be envisioned as micro/nano-electrodes in MEMS based electrochemical sensors and miniaturized energy storage/energy conversion devices.

Pyrolyzed carbon, also known as glass-like carbon, is one of the more popular allotropes of carbon derived through thermal degradation, or carbonization, of organic polymers in inert atmospheres. Over the last couple of decades, pyrolyzed carbon has garnered importance as functional electrode material in electrochemical applications owing to its high isotropy in structural and physical properties [25]. Since organic polymers are used as carbon precursors, different lithography methods can be used to micropattern carbon for MEMS applications. The resistivity values of pyrolyzed carbon films is comparable to that of glassy carbon prepared at same temperature, but with an additional benefit of lower $\mathrm{O} / \mathrm{C}$ ratio [26]. Electrochemical studies have corroborated that the kinetics of redox couple on pyrolyzed carbon films prepared at $700{ }^{\circ} \mathrm{C}$ have glassy carbon-like behavior with good electrochemical reversibility. As the pyrolysis temperature was increased to $1000{ }^{\circ} \mathrm{C}$, pyrolyzed carbon films showed even better electrocatalytic properties than glassy carbon [26]. In addition, pyrolyzed carbon has low capacitance value making it attractive for analytical applications. The electrochemical properties of glassy carbon and pyrolyzed carbon films derived from different precursors are summarized in Table 2.1. 
Table 2.1. Comparison of electrochemical characteristics for glassy carbon and pyrolyzed carbon films derived from different precursors. Table adapted from Ref [2].

\begin{tabular}{|lccc|}
\hline \multicolumn{1}{|c}{ Fabrication Method } & $\begin{array}{c}\text { Resistivity } \\
(\mathbf{m} \mathbf{\Omega} \mathbf{~ c m})\end{array}$ & $\begin{array}{c}\text { Capacitance( } \\
\left.\boldsymbol{\mu} \mathbf{F ~} \mathbf{~ c m}^{2}\right)\end{array}$ & $\begin{array}{c}{ }^{\mathrm{a}} \text { Rate } \\
\text { constant } \mathbf{K}_{\mathbf{0}}\end{array}$ \\
\hline GC-20 Tokai, polished & 4 & 33 & $0.06-0.15$ \\
\hline GC-20, forming gas, $1000^{\circ} \mathrm{C}$ & 4 & 22 & 0.044 \\
\hline Poly(furfuryl alcohol) pyrolysis, $1100^{\circ} \mathrm{C}$ & 10 & -- & -- \\
\hline Poly acrylonitrile pyrolysis, $1020^{\circ} \mathrm{C}$ & 2 & -- & -- \\
\hline Methane pyrolysis, $1100^{\circ} \mathrm{C}$ & -- & 20 & $0.004^{\mathrm{b}}$ \\
\hline Natural gas pyrolysis, $1000^{\circ} \mathrm{C}$ & -- & $>100$ & $0.004-0.015$ \\
\hline Methane pyrolysis & -- & 32 & 0.009 \\
\hline Organic film pyrolysis, $1000^{\circ} \mathrm{C}$ & 4 & -- & -- \\
\hline Sputtered carbon & 35 & 7.5 & $0.024-0.042^{\mathrm{c}}$ \\
\hline PPF forming gas, $1100^{\circ} \mathrm{C}$ & 5.1 & 8.1 & $0.042^{\mathrm{d}}$ \\
\hline
\end{tabular}

${ }^{a}$ Rate constant values $\mathrm{K}_{0}$ were measured against potassium ferricyanide $\left(\mathrm{Fe}(\mathrm{CN})_{6}^{3-/ 4-}\right.$ in $1 \mathrm{M} \mathrm{KCL}$ unless indicated otherwise

b Calculated from volumetric data in Ref 12.

c In $0.5 \mathrm{M} \mathrm{H}_{2} \mathrm{SO}_{4}$

${ }^{\mathrm{d}}$ Corrected for film resistance

\subsection{Carbon Microelectromechanical Systems (C-MEMS) technique}

Carbon Microelectromechanical Systems, also referred to as Carbon-MEMS or C-MEMS, is a term used for set of fabrication methods used to convert pre-patterned photocurable polymers to glass-like carbon microstructures by pyrolysis [27-31]. Different fabrication techniques such as stamping, casting, machining and lithography, among others have been used in this technique [32]. The quality, complexity and final dimensions of the desired carbon structures dictate the technique used for patterning the organic polymer. Currently, the fabrication of Carbon-MEMS devices is principally based on the use of photolithography as the primary tool to pattern the polymeric 
precursors. Illustration of the typical C-MEMS fabrication procedure is shown in Figure 2.3.

(1) Spin photoresist
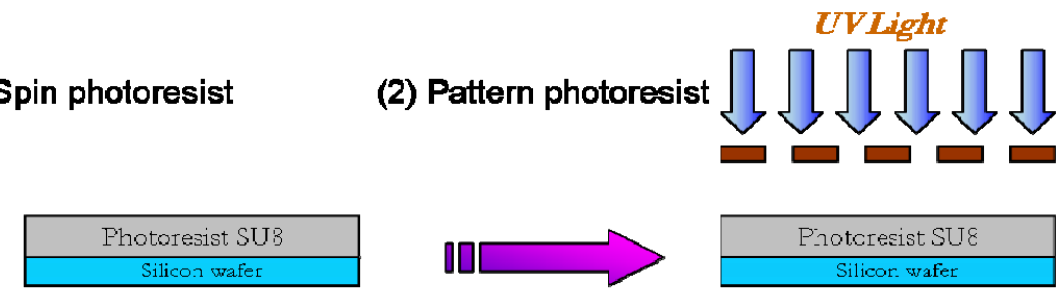

(4) Pyrolysis
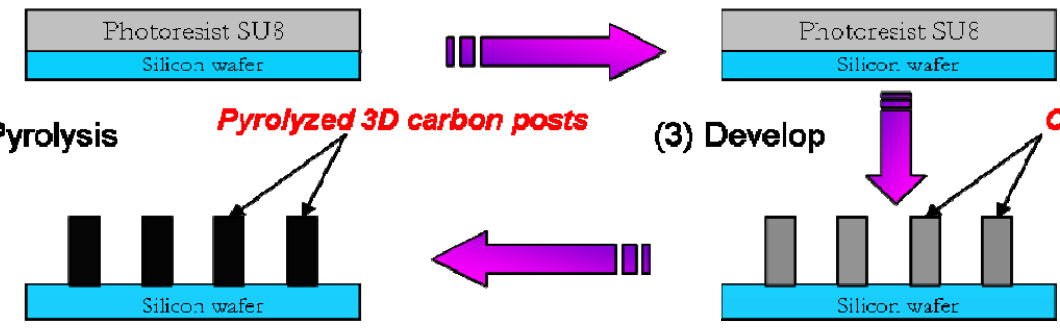

(3) Develop

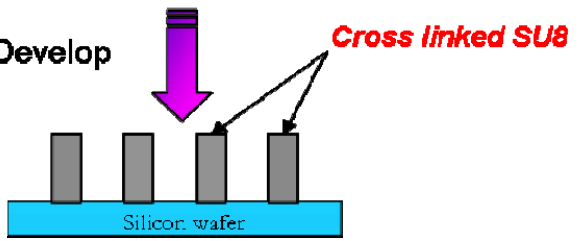

Figure 2.3. Schematic showing the C-MEMS process using a negative tone resist (SU-8) as the precursor.

The existence of standardized photolithography tools and commercial highquality precursors make the fabrication process and the dimensional control highly reproducible. The addition of Next-Generation Lithography (NGL) techniques, such as photo-nanoimprint lithography (NIL), electron beam lithography (EBL), and focused-ion beam (FIB), can further reduce the feature sizes and greatly increase the intricacy of the resulting carbon structures.

The C-MEMS technique received a lot of attention since late 1990s when Schueller and co-workers at Harvard University derived glassy carbon microstructures from carbonizing phenol-formaldehyde resins and furfuryl alcohol-modified phenolic resins. In their process, soft lithography defined polydimethylsiloxane (PDMS) molds were used to pattern the polymers $[33,32]$. This fabrication scheme gave them the flexibility to fabricate different high aspect ratio structures (including free standing lateral comb drives, diffraction gratings and grids) on either curved or flat surfaces [34]. During 
the same decade, initial work emerged regarding the derivation of carbon from photoresists driven by the considerable usage of pyrolyzed photoresists in batteries, electrochemical sensors, biosensors, capacitors and MEMS applications. In 1998, electrochemical studies of carbon films derived from positive photoresists were conducted by Kim et. al. [35,37] and later by Ranganathan and his co-workers [26]. The results showed that the pyrolyzed photoresist films (PPF) have a near-atomic flatness with an electrochemical behavior similar to glassy carbon prepared at same temperature. By 2000, Kinoshita and his team were successful in patterning these carbon films as microelectrodes and studied the influence of the geometry in their electrochemical response [36]. In 2002, Madou's group reported for the first time the use of negative-tone photoresist, $\mathrm{SU}-8$, and polyimide as precursors for preparing carbon micropatterns. It was interesting to note that carbon obtained by using these precursors showed higher resistivity and vertical shrinkage compared to the one synthesized from positive resists. Nevertheless, resistivity from SU-8 carbon was slightly lower than polyimides [38].

The works till this time reporting the preparation of pyrolyzed carbon from organic polymers were only limited to two-dimensional constructs. In 2005, Wang and her co-workers from Madou group were able to demonstrate microstructures with aspect ratios greater than 10 . They used a two-step heating process during pyrolysis which allowed the release of residual oxygen contained in the polymer structures. Previously, one step pyrolysis process caused the precursor to burn rather than pyrolyze, even in an oxygen-free atmosphere due to the insufficient time to degass. Since then, a wide variety of complex high-aspect ratio carbon-MEMS structures, such as pillars, suspended carbon bridges, wires, self-organized bunched posts, plates and networks, have been fabricated. 
A thorough characterization of the structure shrinkage along with electrical properties and shrinkage behavior of both positive and negative resists was conducted by Madou group [39]. They observed that hundreds-of-microns thick features usually shrink approximately $50 \%$ while structures with thickness below $10 \mu \mathrm{m}$ shrink almost by $90 \%$. In addition, they reported a decrease in the resistivity of pyrolyzed carbons as the pyrolysis temperature was increased [40]. In 2006, Malladi et.al. successfully fabricated suspended carbon microstructures by using electron-beam lithography (EBL) [29]. Recently, Wang group at FIU reported the surface engineering of 3D microstructure arrays by integrating functional nanomaterials such as carbon nanotubes [41], graphene [42] and polypyrolle [43]. The addition of nanofeatures to carbon microelectromechanical system (C-MEMS) structures has shown to greatly increase the surface area and enhanced electrochemical functionalities. In addition, Sharma et. al. succeeded in fabricating suspended carbon nanowires $(\mathrm{CNW})$ on micro-fabricated posts by electrospinning of SU-8 photoresist followed by pyrolysis [44]. These CNW selfassembled to connect the posts obviating the need for positioning and integration of carbon nanowires with the underlying microstructures.

Carbon microstructures fabricated by C-MEMS technique have found a variety of applications including on-chip super capacitors, lithium-ion batteries, electrochemical biosensors, DNA detection, bio-fuel cells, cell culturing substrates, and di-electrophoretic electrode arrays for the micromanipulation of micro-and nanoparticles. Some key carbon MEMS/NEMS functions and manufacturing processing still need to be developed to bridge the manufacturing gap between nano and macro features. There is great hope that 
integration of biological components with C-MEMS /NEMS will allow new functional hybrid carbon-biological materials and multilevel devices.

\subsection{Next-Generation Lithography}

Next Generation Lithographies (NGL) are a new breed of lithography techniques postulated as potential replacements to conventional photolithography in the everlasting quest to reduce feature dimensions. Popular NGL techniques include: imprint lithography (which includes thermal-nanoimprint lithography (T-NIL) and photo-nanomprint lithography (P-NIL), extreme ultraviolet lithography (EUVL), charged particle beam lithography based on electrons and ions (such as electron and ion projection techniques) and x-ray lithography. In principle, all these techniques can be used for the manufacture of carbon-MEMS devices. We place an emphasis on imprint lithographies because they represent a more affordable and readily available way to obtain nanostructures for carbon NEMS or nanofluidics applications. EUVL and X-ray lithography may also be used but they are more expensive and are not readily available due to their required infrastructure.

\subsubsection{Nanoimprint lithography}

Imprinting processes are economical due to the simplicity of the equipment involved and the potential for high-throughput. Since the initial development of nanoimprint lithography (NIL), two major approaches have been used in NIL process. The first approach involves imprinting a mold/stamp/template into a thermoplastic or thermoset polymer (Thermal-nanoimprint lithography) and the other into an ultraviolet (UV) light-curable material (Photo-nanoimprint lithography). After imprinting the resist,

a dry anisotropic etch is used to remove the residual resist layer in the compressed area to expose the substrate underneath. For nanoimprinting, once a solid stamp with nano-relief 
features is prepared, it can be used for the replication of many identical surface patterns on the substrate.

Thermal-nanoimprint lithography (T-NIL) was invented in 1994 by Stephen Chou at Princeton University [44]. The resolution of the T-NIL process is directly related to the resolution of the original template/stamp fabrication process. One of the challenges in T-NIL is when imprinting with varying pattern density, results have shown incomplete displacement of the thermoplastic even at elevated temperature and high pressure which could lead to partial pattern transfer. In addition, the use of high temperatures and high pressures limits the ability to achieve the layer-to-layer alignment required for microelectronic device fabrication.

Compared to the T-NIL, photo-nanoimprint lithography (P-NIL) has additional advantages, such as polymerization, which proceeds at room temperature, no pattern shift due to the absence of mismatch of the coefficients of thermal expansion of mold and substrate, high curing speed that reduces the cycle time and the potential for precise layer to layer alignment. The University of Texas (UT)-Austin developed its version of P-NIL also known as step-and-flash imprint lithography (SFIL) in 1998 [45]. The SFIL method operates in a step-and-repeat fashion, rather than serial processing as in the case of T-NIL.

The present day applications of P-NIL process include manufacturing of several emerging technologies, such as micro/nano-optical components, photonic crystals, and nanopatterned magnetic media for future hard disk drives, whereas T-NIL using thermoplastic polymer films is focused on applications such as bio-chips, life sciences, storage media and optical devices. The P- NIL systems which are able to work with 
photocurable polymers are a potential option to fabricate carbon nano-scale structures with controllable dimensions and properties using Carbon-MEMS technique.

\subsection{C-MEMS based Biosensing}

The attractive properties of carbon such as low non-specific adsorption of biomolecules, easy surface modification by various physical/chemical treatments, wide electrochemical window, low cost and excellent biocompatibility has made it a very popular material for biological sensors [46].

Conventionally, screen-printed electrodes are widely used in commercial sensors, but a major bottle neck with screen-printed electrodes is the miniaturization of the sensor. In this regard, C-MEMS technology offers several advantages in MEMS based biological and electrochemical sensor applications such as: (i) the high surface area of the 3D C-MEMS microstructures enables the design of biosensors with improved sensitivity; (ii) since lithography techniques are used, a wide variety of shapes are possible; (iii) the sensing electrodes and contact pads can all be made of carbon in a single process, with linear dimensions ranging from sub-microns to millimeters; (iv) high reproducibility of the material properties within the same temperature range; (v) compared to $\mathrm{Au}$ and $\mathrm{Pt}$ electrodes, carbon has a wider electrochemical stability window; (vi) nanomaterials (such as nanotubes, nanopowders, nanofibers and graphene) can be easily integrated onto 3D microstructure arrays to obtain enhanced mechanical, chemical and electrical properties.

In the last decade, several research groups have reported the use of micro/nanostructures prepared by C-MEMS technique as functional units in biosensing applications. Lee group at KAIST, Korea fabricated an electrochemical impedance biosensor using pyrolyzed carbon film as the sensing electrode for aptamer-based thrombin detection 
[47]. In this work, thrombin aptamer was covalently grafted onto the pyrolyzed carbon surface using carbodiimide-mediated chemistry. The sensor was able to detect thrombin concentrations between $0.5 \mathrm{nM}$ and $500 \mathrm{nM}$ by electrochemical measurement. Another report from the same research group reported the use of carbon nanostructures pyrolyzed at $700{ }^{\circ} \mathrm{C}$ as conducting channel in electrical biosensors [48]. They observed that the conductances of the carbon nanowire channels were increased after target analyte streptavidin interacts with the probe grafted onto the carbon conducting channel. Madou group at UCI which has pioneered the fabrication of 3D carbon microstructures using SU-8 photoresist, constructed amperometric glucose sensors based on high aspect ratio carbon post-microarrays [49]. In this work sensing performance of the glucose sensors with different post-heights and various post-densities was tested and compared. The sensor showed a linear response towards glucose between $0.5 \mathrm{mM}$ to $20 \mathrm{mM}$ range with a response time of about $20 \mathrm{sec}$. The sensitivity per unit footprint substrate area achieved with the $140 \mu \mathrm{m}$ high (aspect ratio around 5:1) carbon post-samples was $2.02 \mathrm{~mA} /(\mathrm{mM}$ $\mathrm{cm}^{2}$ ), which is twice the sensitivity per unit footprint substrate area of the flat carbon films. The same research group investigated the viability of C-MEMS microfabrication technique as a promising approach to create novel platforms for the study of cell physiology [50]. Two cell lines, murine dermal fibroblasts and neuroblastoma spinal cord hybrid cells (NSC-34) were plated onto the substrates, and both cell lines showed preferential adhesion to the selectively plasma-treated regions in carbon films. It was observed that cells were aligned on the carbon electrodes without relying on direct patterning of surface molecules. In 2009, Wakui et. al. an injection type electrohydrodynamic pump (EHD) using pyrolyzed polymer 3D carbon mesh electrodes, 
integrated on one chip [51]. Since multiple 3D carbon mesh electrodes can be prepared using the fabrication process, multiple flow control systems can be produced. Using Fluorinert as sample liquid, the EHD pump could achieve a maximum pressure of about $23 \mathrm{~Pa}$ and maximum flow rate of $400 \mathrm{~nL} / \mathrm{min}$. Construction of a capillary electrophoresis (CE)-based microfluidic device with integrated carbon sensing electrode, obtained by pyrolyzing positive-tone photoresist, was reported by Yaseen et. al. in the same year [52]. The microdevice showed good amperometric response towards morphine and codeine on carbon electrodes. The carbon electrode provided stable background current during the application of a high sensing potential, which is a pre-requisite for sensing molecules that can be only detected at high potentials such as morphine and codine. The application of 3D carbon microstructures was extended to DNA detection as reported by Yang et.al. They studied the surface functionalization of carbon surface using direct amination for covalent attachment of DNA probe followed by target DNA detection [53].

Thus C-MEMS based microarrays are a very promising platform for developing advanced biosensing devices along with other potential applications in miniaturized Liion batteries, on-chip super capacitors and fuel cells.

\subsection{References}

[1] M. E. Weeks, Discovery of the Elements, Easton, Pa.: Journal of Chemical Education, 1968, ISBN: 9780766138728.

[2] F. D. Réaumur, L'art de convertir le fer forgé en acier, et l'art d'adoucir le fer fondu, ou de faire des ouvrages de fer fondu aussi finis que le fer forge, 1722(English translation from 1956), Paris, Chicago.

[3] G. Federico, The cementation of Iron and Steel, 1914, McGraw-Hill Book Company, ISBN: 9781149305690. 
[4] A. Lavoisier, Traite elementaire de chimie.,1789 (English translation in 1790).

[5] P. Shevlin. Formation of atomic carbon in the decomposition of 5tetrazolyldiazonium chloride. 1972, Journal of the American Chemical Society, 94, 1379-1380.

[6] M. J. S. Dewar, D. J. Nelson, P. B. Shevlin, and K. A. Biesiada. Experimental and theoretical investigation of the mechanism of deoxygenation of carbonyl compounds by atomic carbon. 1981, Journal of the American Chemical Society, 103,2802-2807.

[7] T.W. Ebbesen, ed. Carbon nanotubes-preparation and properties. 1997, Boca Raton, Florida: CRC Press. ISBN: 0849396026.

[8] H. W. Kroto, J. R. Heath, S. C. O'Brien, R. F. Curl, and R. E. Smalley. C60: Buckminsterfullerene. 1985, Nature, 162-163.

[9] C. Frondel, U. B. Martin. Lonsdaleite, a hexagonal polymorph of diamond. 1967, Nature, 587-589.

[10] R. J. Lagow, J. J. Kampa, H-C Wei, S. L. Battle, J. W. Genge, D. A. Laude, C. J. Harper, R. Bau, R. C. Stevens, J. F. Haw, and E. Munson. Synthesis of linear acetylenic carbon: The sp carbon allotrope. 1995, Science, 362-367.

[11] A. V. Rode, E. Gamalay, A. G. Christy, J. Fitz Gerald, S. T. Hyde, R. G. Elliman, B. Luther-Davies, A. I. Veinger, J. Androulakis, and J. Giapintzakis. Unconventional magnetism in all-carbon nanofoam.2004, Physical Review B, 70(5):0544071-0544079.

[12] J. Robertson. Hard amorphous (diamond-like) carbons. 1991, Progress in Solid State Chemistry, 21,199-333.

[13] D. B. Ratner, Pyrolytic carbon. 2004, In Biomaterials science: an introduction to materials in medicine. Academic Press., ISBN:0125824637.

[14] A. K. Geim, K. S. Novoselov, The rise of graphene, 2007, Nature Materials, 6 , 183-191.

[15] http://en.wikipedia.org/wiki/File:Eight_Allotropes_of_Carbon.png.

[16] N. L. Pocard, D. C. Alsmeyer, R. L. McCreery, T. X. Neenan, M. R. Callstrom. Doped glassy carbon: A new material for electrocatalysis. 1992, Journal of Materials Chemistry, 2, 771-784. 
[17] M. J. Madou, Fundamentals of microfabrication: The science of miniaturization. 1998, CRC-Press, ISBN: 0849394511.

[18] G.E. Hak, The MEMS handbook. 2001, Boca Raton: CRC press, ISBN: 0849321069

[19] R. P. Ribas, J. L. Leclercq, J. M. Karam, B. Courtois, P. Viktorovitch, Bulk micromachining characterization of $0.2 \mu \mathrm{m}$ HEMT MMIC technology for GaAs MEMS design, 1998, Materials Science and Engineering, B: Solid-State Materials for Advanced Technology, 51, 267-273.

[20] M. Datta, M. W. Pruessner, D. P. Kelly, R. Ghodssi, Design of MEMS-tunable novel monolithic optical filters in InP with horizontal bragg mirrors. 2004, SolidState Electronics, 48, 1959-1963.

[21] M. Gromova, K. Baert, C.Van Hoof, A. Mehta, A. Witvrouw, The novel use of low temperature hydrogenated microcrystalline silicon germanium (SiGe:H) for MEMS applications. 2004, Microelectronic Engineering, 76, 266-271.

[22] S. Guan, R. B. Nielsen, Rapid scan Fourier transform detection of a frequency encoded quartz crystal microbalance array. 2003, Review of Scientific Instruments, 74, 5241-5248.

[23] M. Evans, C. Sewter, E. Hill, An encoded particle array tool for multiplex bioassays. 2003, Assay and Drug Development Technologies, 1, 199-207.

[24] C. Wang, M. Madou, From MEMS to NEMS with carbon. 2005, Biosensors and Bioelectronics, 20, 2181-2187.

[25] A. M. Lyons, Photodefinable carbon films: electrical properties. 1985, Journal of Non-Crystalline Solids, 70, 99-109.

[26] S. Ranganathan, R. McCreery, S. Majji, M. Madou, Photoresist-Derived Carbon for Microelectromechanical Systems and Electrochemical Applications. 2000, J. Electrochem. Soc., 147, 277-282.

[27] K. S. Ma, G. Jia, C. Wang, M. Madou, Fabrication of nanoscale carbon structures by C-NEMS technology. 2005, Proceedings of NSTI-Nanotech, 2, 151-154,

[28] O. J. A. Schueller, S. T. Brittain, C. Marzolin, G. M. Whitesides, Fabrication and characterization of glassy carbon MEMS. 1997, Chemistry of Materials, 9, 13991406

[29] K. Malladi, C. Wang, M. Madou, Microfabrication of suspended C-MEMS structures by EB writer and pyrolysis method. 2006, Carbon, 44, 2602-2607 
[30] S. Konishi, M. Liger, T. A. Harder, Y. C. Tai, Parylene-Pyrolyzed carbon for MEMS application. 2004, Proceedings of IEEE MEMS, 161-164

[31] C. Wang, R.Zaouk, M. Madou, Local CVD of carbon nanofibers from photoresist. 2006, Carbon, 44, 3073-3077.

[32] O. J. A Schueller, S. T. Brittain, G. M. Whitesides, Fabrication of glassy carbon microstructures by soft lithography. 1999, Sensors and Actuators A, 72, 125-139

[33] O. J. A. Schueller, S. T. Brittain, G. M. Whitesides. Fabrication of glassy carbon microstructures by pyrolysis of microfabricated polymeric precursors. 1997, Advanced Materials, 9, 477-480, 1997

[34] X.-M. Zhao, Y. Xia, O. J. A. Schueller, D. Qin, G. M. Whitesides. Fabrication of microstructures using shrinkable polystyrene films..1998, Sensors and Actuators A: Physical, 65, 209-217.

[35] J. Kim, X. Song, K. Kinoshita, M. Madou, R. White. Electrochemical studies of carbon films from pyrolyzed photoresist.1998, Journal of the Electrochemical Society, 45, 2314-2319.

[36] R. Kostecki, X. Song, K. Kinoshita. Influence of geometry on the electrochemical response of carbon interdigitated microelectrodes.2000, Journal of the Electrochemical Society, 147,1878-1881.

[37] S. Ranganathan, L. McCreery. Electroanalytical performance of carbon films with near-atomic flatness.2001, Analytical Chemistry, 73, 893-900.

[38] A. Singh, J. Jayaram, M. Madou, S. Akbara. Pyrolysis of negative photoresists to fabricate carbon structures for microelectromechanical systems and electrochemical applications. 2002, Journal of the Electrochemical Society, 149, E78-E83.

[39] C. Wang, G. Jia, L. H. Taherabadi, M. J. Madou. A novel method for the fabrication of high-aspect ratio C-MEMS structures.2005, Journal of Microelectromechanical Systems, 14,348-358.

[40] B. Park, L. Taherabadi, Ch. Wang, J. Zoval, and M. Madou. Electrical properties and shrinkage of carbonized photoresist films and the implications for carbon microelectromechanical systems devices in conductive media. 2005, Journal of the Electrochemical Society, 152, J136-J143.

[41] W. Chen, M. Beidaghi, V. Penmatsa, K. Bechtold, L. Kumari, W.Z. Li, C. Wang, Integration of Carbon Nanotubes to C-MEMS for On-chip Supercapacitors. 2010, Nanotechnology, IEEE Transactions on, 9, 734-739. 
[42] V. Penmatsa, T. Kim, M. Beidaghi, H. Kawarada, Z. Wang, L. Gu, C. Wang, Three-dimensional graphene nanosheets encrusted carbon micropillar arrays for electrochemical sensing. 2012, Nanoscale, DOI:10.1039/C2NR30161J.

[43] W. Chen, M. Beidaghi, V. Penmatsa, K. Bechtold, L. Kumari, W.Z. Li, C. Wang, Integration of Carbon Nanotubes to C-MEMS for On-chip Supercapacitors. 2010, Nanotechnology, IEEE Transactions on, 9, 734-739.

[44] M. Beidaghi, C. Wang, Micro-supercapacitors based on Three Dimensional Interdigital Polypyrolle/C-MEMS Electrodes.2011, Electrochimica Acta, 95089514.

[45] M. Colburn, S. Johnson, M. Stewart, S. Damle, T. Bailey, B. Choi, M. Wedlake, T. Michaelson, S.V. Sreenivasan, J.G. Ekerdt, C.G. Willson. Step and flash Imprint lithography: A new approach to high resolution patterning. 1999, Proc. SPIE, 3676(I), 379-390

[46] S. Sotiropoulou, V. Gavalas, V. Vamvakaki, N.A Chaniotakis, Novel carbon materials in biosensor systems. 2002, 18, 211-215.

[47] J. A. Lee, S. Hwang, J. Kwak, S. I. Park, S. S. Lee, K-C Lee, An electrochemical impedance biosensor with aptamer-modified pyrolyzed carbon electrode for labelfree protein detection. 2008, Sensors and Actuators B, 129, 372-379.

[48] J. A. Lee, K-C. Lee, S. I. Park, S. S. Lee, The fabrication of carbon nanostructures using electron beam resist pyrolysis and nanomachining processes for biosensing applications. 2008, 19, 215302 (pp7).

[49] H. Xu, K. Malladi, C. Wang, L. Kulinsky, M. Song, M. Madou, Carbon postmicroarrays for glucose sensors. 2008, 23, 163-1644.

[50] G. T. Teixidor, R. A. Gorkin, P. P. Tripathi, G. S. Bisht, M. Kulkarni, T. K. Maiti, T. K. Battacharyya, J. R. Subramaniam, A. Sharma, B. Y. Park, M. Madou, Carbon microelectromechanical systems as a substratum for cell growth. 2008, Biomed. Mater., 3, 034116 (8pp).

[51] D. Wakui, N. ImaiI, Y. Nagaura, H. Sato, T. Sekiguchi, S. Konishi, S. Sholiland, T. Homma, EHD micro pump using pyrolyzed polymer $3 D$ carbon mesh electrodes. 2009, J. IEEE, 499-502.

[52] A. A. D. B- Yaseen, Fabrication and characterization of fully integrated microfluidic device with carbon sensing electrode for the analysis of selected biomedical targets. 2009, IEEE Sensors Journal, 9, 81-86. 
[53] J.H. Yang, V. Penmatsa, S. Tajima, H. Kawarada, C. Wang, Direct amination on 3-dimensional pyrolyzed carbon micropattern surface for DNA detection. 2009, Materials Letters, 63, 2680-2683 


\section{CHAPTER 3}

\section{FABRICATION OF 3D POROUS CARBON MICROARRAYS}

\subsection{Introduction}

Carbon and its allotropes have received a lot of research interest owing to their attractive physiochemical characteristics such as good electrical conductivity, chemical inertness, low background capacitance, biocompatibility and low cost [1]. In addition, carbon surface can be also tailored by various physical/chemical treatments making it an attractive material for diverse applications in biological, mechanical and energy storage devices [2-4]. At present, porous carbon materials with their commensurate increase in surface area are extensively used for various applications such as catalyst support for biomolecules, electrodes for biosensors, electrochemical double layer capacitors, fuel cells and gas separation [5,6]. Conventional synthesis techniques such as thermal decomposition of natural precursors [7], catalytic activation of carbon precursors using metal salts and pyrolysis of polymer blends are commonly used to produce porous carbon [8]. A challenging aspect of the above mentioned techniques is the synthesis of ordered porous structure since the resultant carbon has porosity distribution dependent on the precursor material. For synthesizing ordered porous carbon, several approaches using solid templates have been reported, where a porous template filled with a carbon precursor is first carbonized followed by the removal of the template $[9,10]$. As a result, the precursor is converted to carbon and the host template is transferred into pores. More recently, Gogotsi and his coworkers synthesized carbide-derived carbons by hightemperature chlorination of carbides. In this approach, the metals and metalloids in the precursor material are removed as chlorides, leaving behind nanoporous carbon with 50- 
$80 \%$ open pore volume [11]. Although mesoporous carbons synthesized by the above techniques have wide range pore distribution, they need to be casted as electrodes using a binder so patterning them at the microscale is arduous and challenging.

In our previous research, we employed carbon-microelectromechanical systems (C-MEMS) technique to fabricate three-dimensional carbon microstructures. Considering the fact that photoresist can be precisely patterned using photolithography, the electrodes fabricated by this technique yield higher resolution and reproducible structures compared to the traditional screening printing. Increasing the surface area of the carbon microelectrodes by introducing porosity could deem helpful in the further improvement of sensitivity and device performance of C-MEMS based functional devices such as onchip supercapacitors, enzymatic biofuel cells and biosensors.

In this chapter, two surface engineering strategies for producing porous carbon micropillars, i.e. by using modified C-MEMS process using a block copolymer as porogen and oxygen RIE treatment of traditional C-MEMS structures are reported.

\subsection{Materials and Methods}

Pluronic F127 (molecular weight 12,500 and PEO content of 70\%), was obtained from Sigma Aldrich, USA. NANO ${ }^{\mathrm{TM}}$ SU-8 100, NANO ${ }^{\mathrm{TM}}$ SU-8 25 and NANO ${ }^{\mathrm{TM}}$ SU-8 developer were purchased from Microchem, USA.

\subsubsection{Using F127 as Porogen}

\subsubsection{Precursor Preparation}

Different photoresist precursors $(2.5 \%, 5 \%, 7.5 \%$ and $10 \%$ F127 weight/weight with SU-8) were prepared by ultrasonicating suitable amount of F127 dissolved in $5 \mathrm{ml}$ acetone for $10 \mathrm{~min}$ and then mixing with $10 \mathrm{~g}$ of $\mathrm{NANO}^{\mathrm{TM}}$ SU-8 100 photoresist. This 
photoresist precursor mixture was thoroughly mixed using a pipette for $15 \mathrm{~min}$ and left overnight to evaporate acetone. A similar procedure was followed to make $\mathrm{NANO}^{\mathrm{TM}}$ SU-8 $25+$ F127 precursor to fabricate two-dimensional porous carbon electrodes for electrochemical characterization.

\subsubsection{Modified C-MEMS Process}

Silicon oxide wafers (4 in. size, (100)-oriented, n-type) were spin cleaned by acetone and methanol and baked on a hotplate at $150{ }^{\circ} \mathrm{C}$ for $5 \mathrm{~min}$ to evaporate any moisture. For the SU-8 coating, a typical spin coating process was carried out using a photoresist spinner (Headway research ${ }^{\mathrm{TM}}$ ) at $500 \mathrm{rpm}$ for $12 \mathrm{sec}$ then $3000 \mathrm{rpm}$ for $30 \mathrm{sec}$. Following the spin coating process, the photoresist was soft baked at $65^{\circ} \mathrm{C}$ for 10 min and hard baked at $95^{\circ} \mathrm{C}$ for $30 \mathrm{~min}$ in a lindberg box oven, in order to evaporate any remaining solvents and harden the photoresist. Photoresist was then exposed by a broadband mercury lamp for $105 \mathrm{sec}$ using an OAI Hybralign contact aligner (light intensity, $12 \mathrm{~mW} / \mathrm{cm}^{2}$ ) to crosslink polymer chains in the photoresist. Post expose bake was carried out at $65^{\circ} \mathrm{C}$ for $1 \mathrm{~min}$ and $95^{\circ} \mathrm{C}$ for $3 \mathrm{~min}$ to further harden the crosslinked photoresist. The samples were developed using NANO ${ }^{\mathrm{TM}} \mathrm{SU}-8$ developer (Microchem, USA) for 10 min to wash away the unexposed photoresist. These samples were then rested in Milli-Q water bath and annealed in a box oven at $80^{\circ} \mathrm{C}$ for $4 \mathrm{hr}$. The annealing temperature was chosen to exceed the gelation temperature of F127. Subsequent vacuum treatment was performed in a conventional vacuum dessicator for $4 \mathrm{hr}$. Carbonization of the polymer micropillars was conducted in a Lindberg alumina-tube furnace by a twostep process. The samples were initially heated at $2{ }^{\circ} \mathrm{C} / \mathrm{min}$ rate from room temperature to $400{ }^{\circ} \mathrm{C}$, then holding at $400{ }^{\circ} \mathrm{C}$ for 40 min followed by ramping to $900{ }^{\circ} \mathrm{C}$ at $5{ }^{\circ} \mathrm{C} / \mathrm{min}$ 
rate. Next, the samples were kept at $900{ }^{\circ} \mathrm{C}$ for $60 \mathrm{~min}$ and then cooled down to room temperature. The whole carbonization process was conducted in forming gas $\left(95 \% \mathrm{~N}_{2}+\right.$ $5 \% \mathrm{H}_{2}$ ) atmosphere with constant gas flow rate at $500 \mathrm{sccm}$. A typical modified C-MEMS fabrication process is illustrated in Figure 3.1.

(a) Spin coating

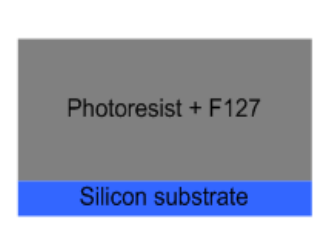

(e) Pyrolysis (b) Patterning

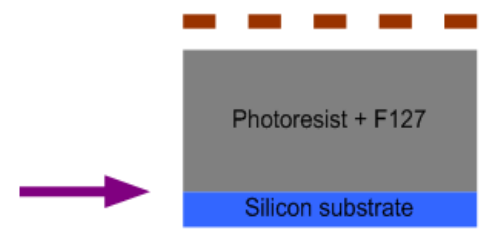

(d) Vaccum
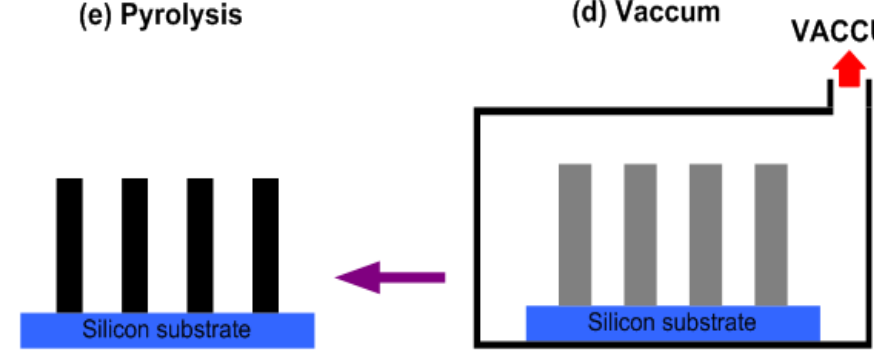

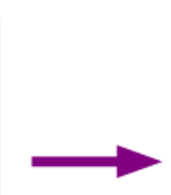

.

m

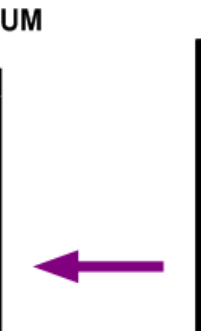

(c) Developing

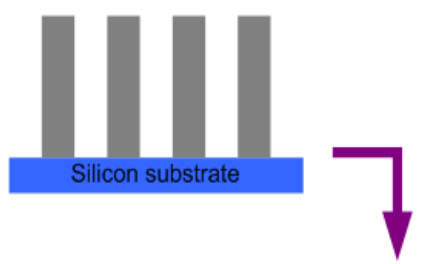

(d) Gelation of F127

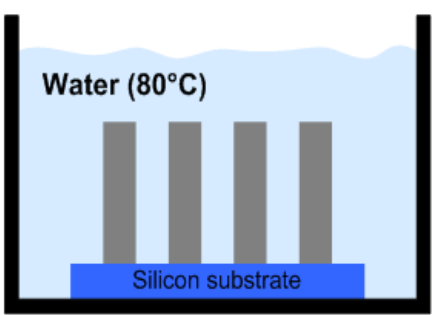

Figure 3.1. Schematic of the fabrication process: (a) spin coating the photoresist + F127 precursor to obtain a uniform film on the substrate, (b) patterning the photoresist precursor using UV light, (c) the resultant patterned array of three dimensional microstructures after developing using suitable developer, (d) soaking three-dimensional microstructures in water bath at $80{ }^{\circ} \mathrm{C}$ to induce gelation of F127, (e) subsequent vacuum treatment to eliminate bubbles formed, and (f) carbonizing the polymer microstructures under forming gas atmosphere at high temperature.

\subsubsection{Two-dimensional Carbon Electrodes for Electrochemical Characterization}

Thin film porous carbon electrodes prepared on silicon dioxide substrates were used as working electrodes for electrochemical measurements. Fabrication process of the porous carbon film electrodes is very similar to the above described fabrication procedure for micropillars. In this case SU-8 25 mixed with different concentrations of F127 was 
spincoated onto the silicon oxide substrates at $500 \mathrm{rpm}$ for $12 \mathrm{sec}$ then at $3000 \mathrm{rpm}$ for 30sec. The resist layer was then soft baked at $65^{\circ} \mathrm{C}$ for $3 \min$ and $95^{\circ} \mathrm{C}$ for $7 \mathrm{~min}$ on a hot plate before exposing with a dose of $720 \mathrm{mWcm}^{2}$. A post expose bake, on a hot plate, was performed for 1 min at $65{ }^{\circ} \mathrm{C}$ and $3 \min$ at $95{ }^{\circ} \mathrm{C}$. These polymer structures were carbonized using the same heating cycle described above. To complete the working electrode fabrication, an electrical contact was established by using a silver wire and copper tape. The undesired active electrode surface was masked with epoxy.

\subsubsection{Characterization}

Thermal behavior of SU-8 and F127 mixed SU-8 was investigated by DSC-2910 differential scanning calorimetry and TGA-2950 thermogravimetric analysis (both from Thermal Analysis Instruments) under argon atmosphere. The heating cycle employed for both DSC and TGA test is same as the carbonization cycle. JEOL JSM-6335 Scanning Electron Microscopy (SEM) was utilized to investigate the surface morphology of the porous carbon micropillars. To avoid charging of SU-8, a thin gold film was evaporated onto photoresist micropillar samples before carbonization. The pore size distribution was also characterized by Veeco Nanoscope AFM.

In order to quantify the effective surface area of different electrodes, electrochemical measurements were performed with a potentiostat $(\mathrm{CH}$ Instruments, Model 263A) in a three electrode setup at room temperature using $10 \mathrm{mM}$ potassium ferricyanide and $3 \mathrm{M}$ potassium chloride solution as the electrolyte. Platinum wire was used as the counter electrode and all potentials were measured against an $\mathrm{Ag} / \mathrm{AgCl}$ reference electrode. 


\subsubsection{Using Oxygen RIE treatment}

MARCH CS-1217 RIE system was used to treat the pyrolyzed carbon surface with oxygen plasma. This system has a parallel plate reactor equipped with $13.65 \mathrm{MHz}$ RF source. The gas line for oxygen was completely evacuated before the process to remove any moisture. Table 3.1 lists the conditions of the oxygen plasma treatment of the 3D carbon posts.

Table 3.1. Processing conditions of oxygen plasma treatment on carbon micropillars

\begin{tabular}{|cccc|}
\hline Pressure (mTorr) & Power (Watts) & Gas flow (sccm) & Time (sec) \\
\hline 400 & 100 & 100 & $20,40,60,80$ \\
\hline 400 & 150 & 10 & 60 \\
\hline 100 & 100 & 8 & 60 \\
\hline 100 & 150 & 8 & 60 \\
\hline 100 & 150 & 10 & 60,140 \\
\hline
\end{tabular}

\subsection{Results and Discussion}

\subsubsection{Using F127 as Porogen}

\subsubsection{Thermal Characterization}

For fabrication of the porous structure, a block copolymer F127 consisting of poly ethylene oxide (PEO) and poly propylene oxide (PPO) monomers was used as porogen due to its surfactant nature [14]. PPO is hydrophilic below $15^{\circ} \mathrm{C}$, but it turns hydrophobic at elevated temperatures due to its diminishing hydrogen bonding with water. In contrast, PEO is predominantly hydrophilic within the temperature range $0-100{ }^{\circ} \mathrm{C}$ [14]. To compare the thermal behavior of F127 mixed SU-8 mixture with pure SU-8, DSC and TGA studies were conducted under the same carbonization cycle. DSC curves indicated 
in Figure 3.2 show a solvent loss cycle (endothermal process) at low temperatures. During this cycle, residual acetone in the F127 and SU-8 mixture was also evaporated. The exothermal reaction starting at about $160^{\circ} \mathrm{C}$ in the SU-8 curve could be attributed to the decomposition of the photoactive compound. The subsequent exothermal reaction starting at $190{ }^{\circ} \mathrm{C}$ is due to thermal crosslinking observed in epoxy-based polymers like SU-8 [15]. As evidenced from the sharp endothermic peak, F127 starts to form a gel from $60^{\circ} \mathrm{C}$. But for the mixed photoresist precursor $(\mathrm{F} 127+\mathrm{SU}-8)$, due to the presence of F127, the decomposition temperature of the photoinitiator in the F127 and SU-8 mixture is believed to be shifted. In this case, the broad endothermal process with peak around $140{ }^{\circ} \mathrm{C}$ and its onset around $60{ }^{\circ} \mathrm{C}$ could be the combination of the decomposition of the photoactive compound and the gelation of F127. This broad peak ranging from $60{ }^{\circ} \mathrm{C}$ to $200{ }^{\circ} \mathrm{C}$ is caused by the increased dehydration of the EO chains with the increase of temperature. The increase in the hydrophobicity could be the driving force for micellemicelle aggregation. It is reported that the thermal gelation of F127 occurred essentially from the packing of spherical micelles [16]. The other exothermal peaks above $200{ }^{\circ} \mathrm{C}$ in the DSC curves show the onset of different reactions suggesting the gas evolution as the carbonization of the polymer occurs [17]. It is important to note that the exact reactions are hard to confirm since the exact chemical composition of the SU-8 photoresist is not given in the datasheet. 


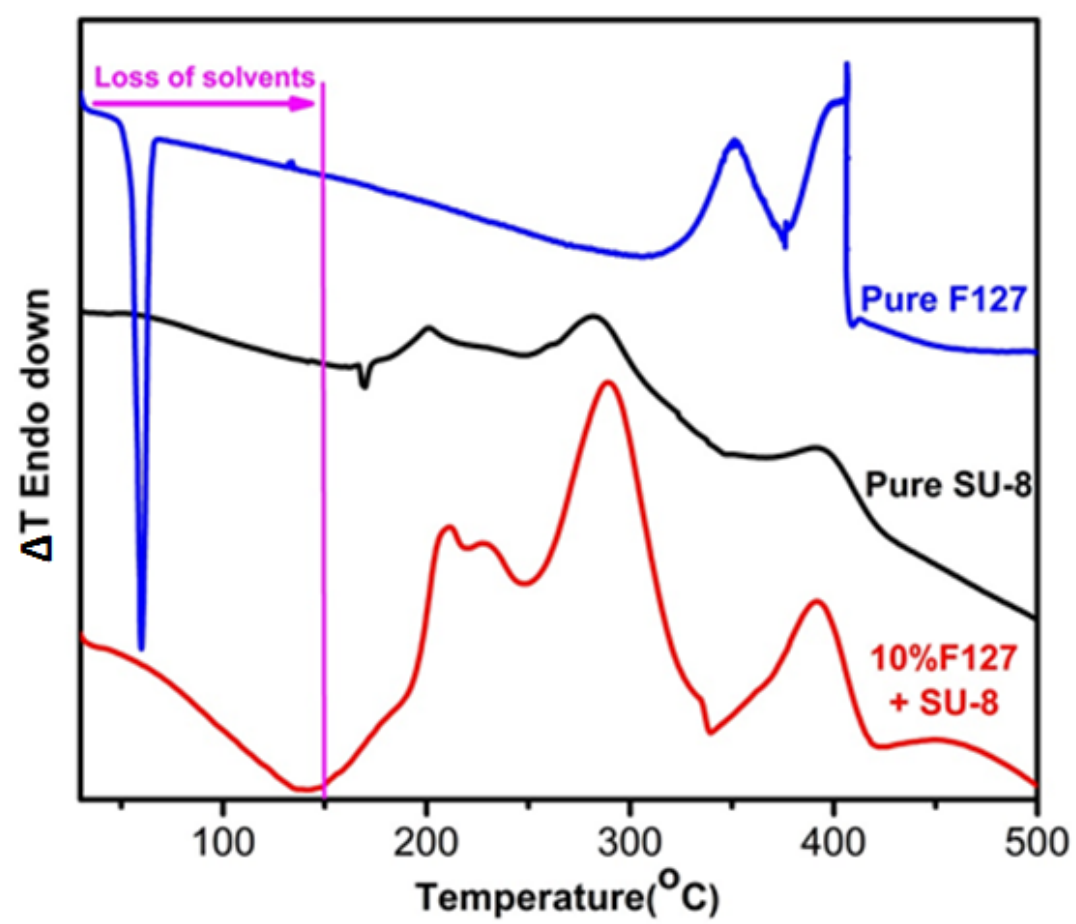

Figure 3.2. Thermal behavior of pure SU-8, F127 mixed with SU-8 and pure F127 investigated from $30{ }^{\circ} \mathrm{C}$ to $500{ }^{\circ} \mathrm{C}$ by DSC

Additionally, thermal stability of the SU-8 samples with and without F127 was examined by TGA as shown in Figure 3.3. It can be observed that there is a $25 \%$ weight loss starting at $100{ }^{\circ} \mathrm{C}$ in both TGA curves due to moisture and solvent evaporation. The decomposition occurred gradually up to $350{ }^{\circ} \mathrm{C}$ followed by a sharp weight loss at around $350-450^{\circ} \mathrm{C}$. Eventually the polymer is completely carbonized from $600{ }^{\circ} \mathrm{C}$ to $900{ }^{\circ} \mathrm{C}$. F127 is also completely decomposed by $400{ }^{\circ} \mathrm{C}$ suggesting that there is no presence of F127 after pyrolysis at $900{ }^{\circ} \mathrm{C}$. Therefore, the as-prepared porous carbon micropillars are completely free of F127. 


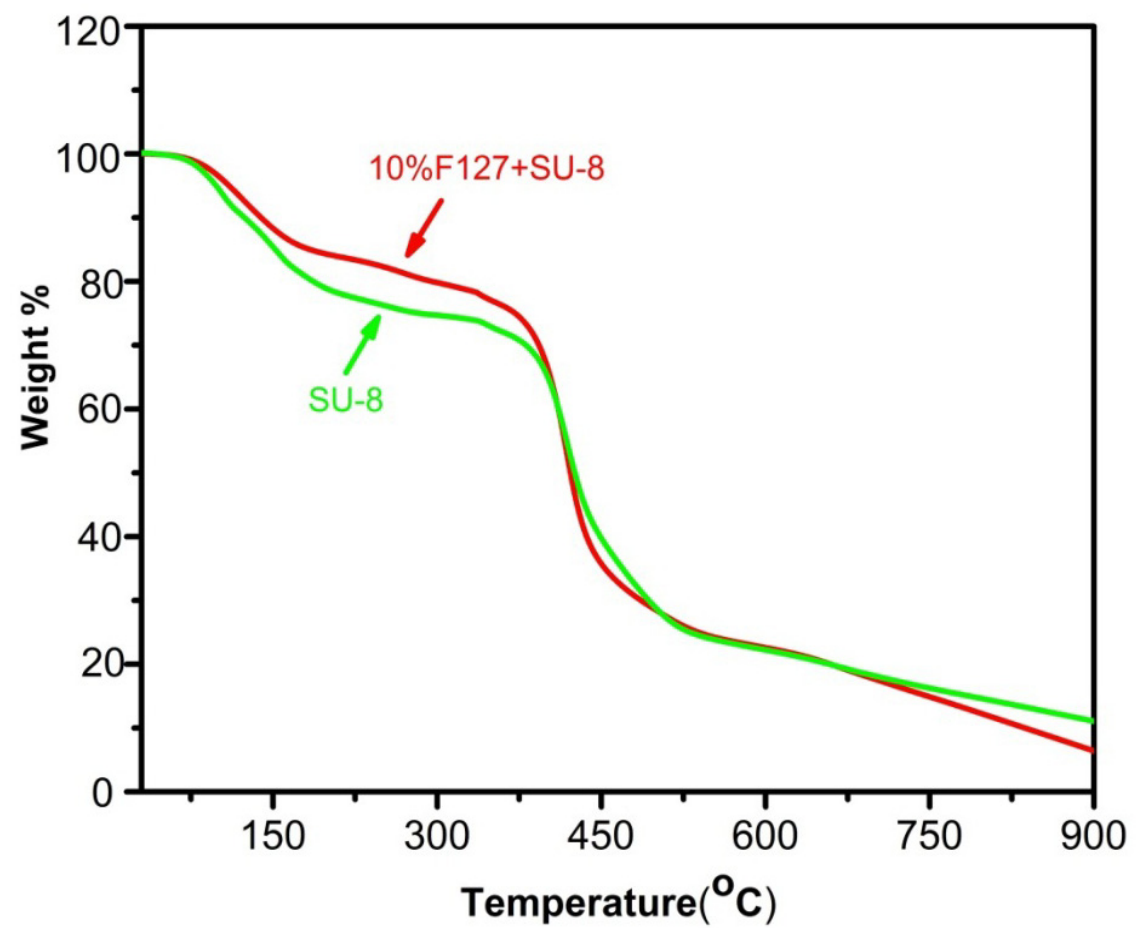

Figure 3.3. Thermal behavior of pure SU-8, F127 mixed with SU-8 and pure F127 investigated from $30{ }^{\circ} \mathrm{C}$ to $900{ }^{\circ} \mathrm{C}$ by TGA

\subsubsection{Morphology Characterization}

The SEM images of photoresist micropillar arrays after annealing in water and vacuum treatment are shown in Figure 3.4 (a) and (b). During the fabrication process, annealing the polymer micropillars in water serves two purposes. By choosing the annealing temperature higher than the gelation temperature of F127, the formation of a thin porous film on the surface was accommodated. In addition, the hydrophobic functional groups in F127 tend to repel the water molecules. As the distance between the water molecules increases, there will be a consequent decrease in the surface tension causing bubble formation. The bubbles on the surface are later transformed into pores by vacuum treatment. Unlike the case of pure SU-8 micropillars where the surface is usually smooth [12], the surface of the micropillar made from F127 mixed SU-8 has a thin 
porous film indicating the gelation of F127. Higher magnification SEM images of the surface shown in Figure 3.4(b) indicate an average pore diameter of few tens of nanometers. Also, towards the bottom portion of the micropillars some residues can be found on the substrate which could be the flux of F127 gel during the annealing step.

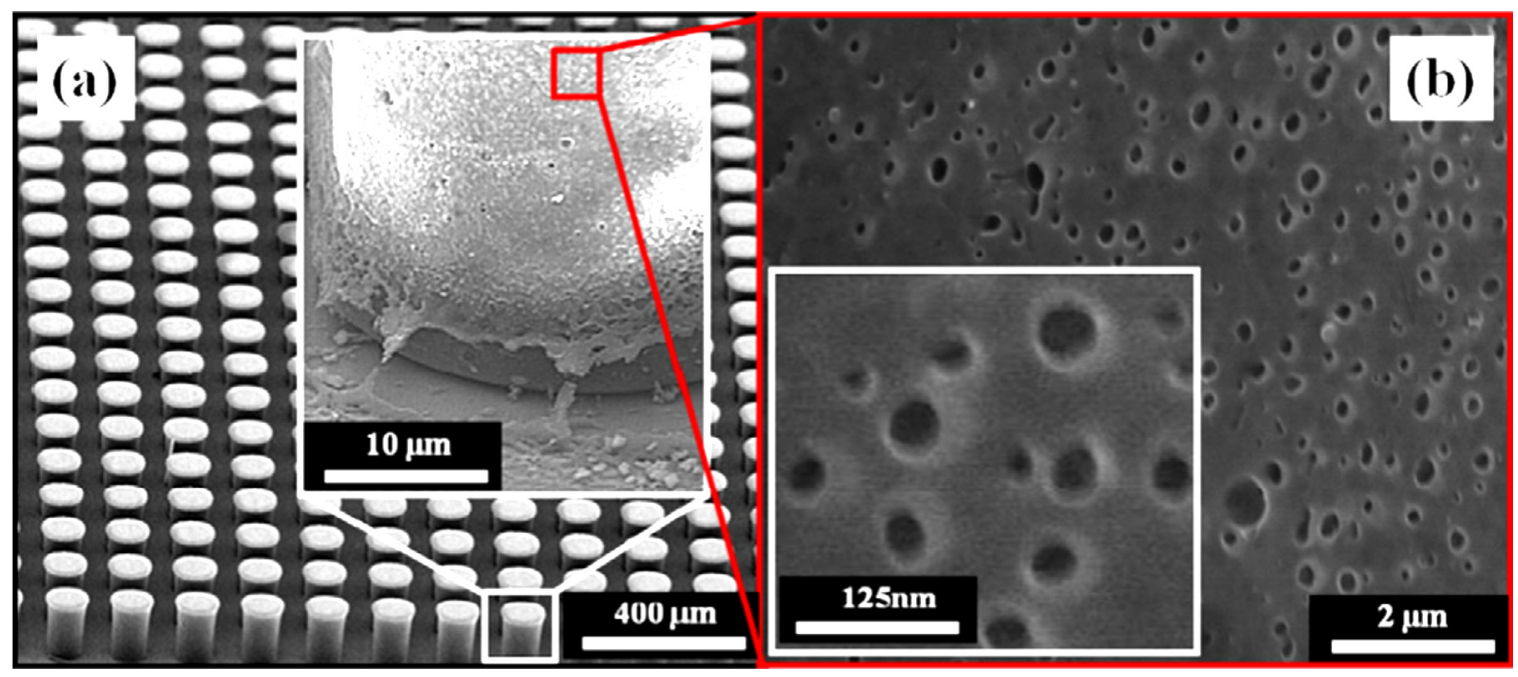

Figure 3.4. SEM images of three-dimensional micropillars derived from F127 mixed SU-8 (tilted at $60^{\circ}$ ) precursor after water annealing and vacuum treatment: (a) micropillar arrays with a single micropillar in the inset and (b) high magnification SEM image of the surface showing porous morphology.

Figure 3.5 shows the corresponding SEM pictures after carbonizing the photoresist micropillars. From these images it can be observed that there is a significant volume change of the micropillars before and after carbonization. It should be noted that photoresist undergoes significant out-gassing during the carbonization process. The removal of non-carbon species during carbonization induces densification of the polymer micropillars and thus accounts for the volume shrinkage [18]. The bottom portion of the carbon microstructures has less shrinkage compared to the top part mainly due to adhesive force at the photoresist and substrate interfaces [19]. The capping effect at the 
top portion of the carbon micropillars is mainly because of a slightly overexposure which was well discussed in our previous work [12]. High magnification SEM images of the carbon surface shown in Figure 3.5(b) illustrate a very jagged surface with mainly mesoporous features. As expected, the mean diameter of the pores coming from outgassing of the volatile components in photoresist decreased after carbonization due to the shrinkage and densification during polymer to carbon transformation.

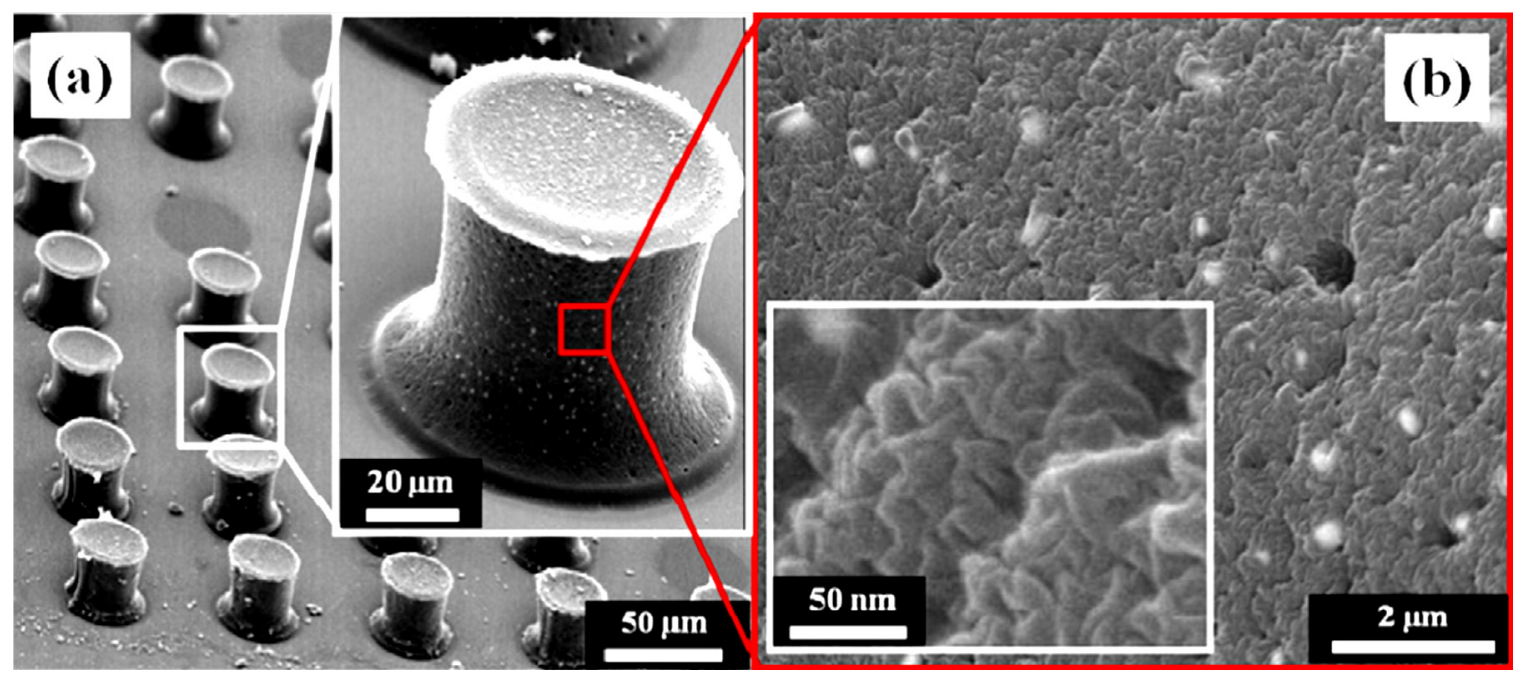

Figure 3.5. SEM images of three-dimensional microstructure derived fromF 127 mixed SU-8 after carbonization: (a) porous carbon micropillar arrays and (b) high magnification SEM images of the porous carbon film.

\subsubsection{Electrochemical Characterization}

A quantitative surface area measurement technique called "electrochemical BET' [20] based on cyclic voltammetry was used to quantify the total surface area of the porous carbon electrodes. Since fabricating carbon micropillars from SU-8 with different concentration of F127 is very challenging to achieve exactly the same total surface areas, for quantitative analysis purpose, two-dimensional porous carbon thin film electrodes were selected instead of micropillar electrodes in order to maintain the same projected 
footprint surface areas exposed to the electrolyte. Figure 3.6 shows the cyclic voltammetry curves of porous carbon thin film electrodes derived from photoresist mixtures with five different concentrations of porogen. The working electrode setup used in the analysis is shown in Figure 3.6 inset. Modified Randles-Sevchik equation for quasi reversible reactions was used to calculate the increase in effective surface area $\left(\mathrm{A}_{\mathrm{eff}}\right)[21]$.

$$
\mathrm{i}_{\mathrm{p}}=-3.01 \cdot 10^{5} \mathrm{n}^{3 / 2} \alpha^{1 / 2} \mathrm{D}_{0}{ }^{1 / 2} \mathrm{~A}_{\mathrm{eff}} \mathrm{c}_{0}|\mathrm{v}|^{1 / 2}
$$

Where $i_{p}$ is the peak current, $n$ is the number of electrons appearing in halfreaction for the redox couple, a is the transfer coefficient, $\mathrm{D}_{0}$ is the diffusion coefficient of the analyte $\left(\mathrm{cm}^{2} / \mathrm{s}\right), \mathrm{A}_{\text {eff }}$ is the electrode area $\left(\mathrm{cm}^{2}\right), \mathrm{c}_{0}$ is the analyte concentration and $\mathrm{m}$ is the rate of the potential sweep $(\mathrm{V} / \mathrm{s})$.

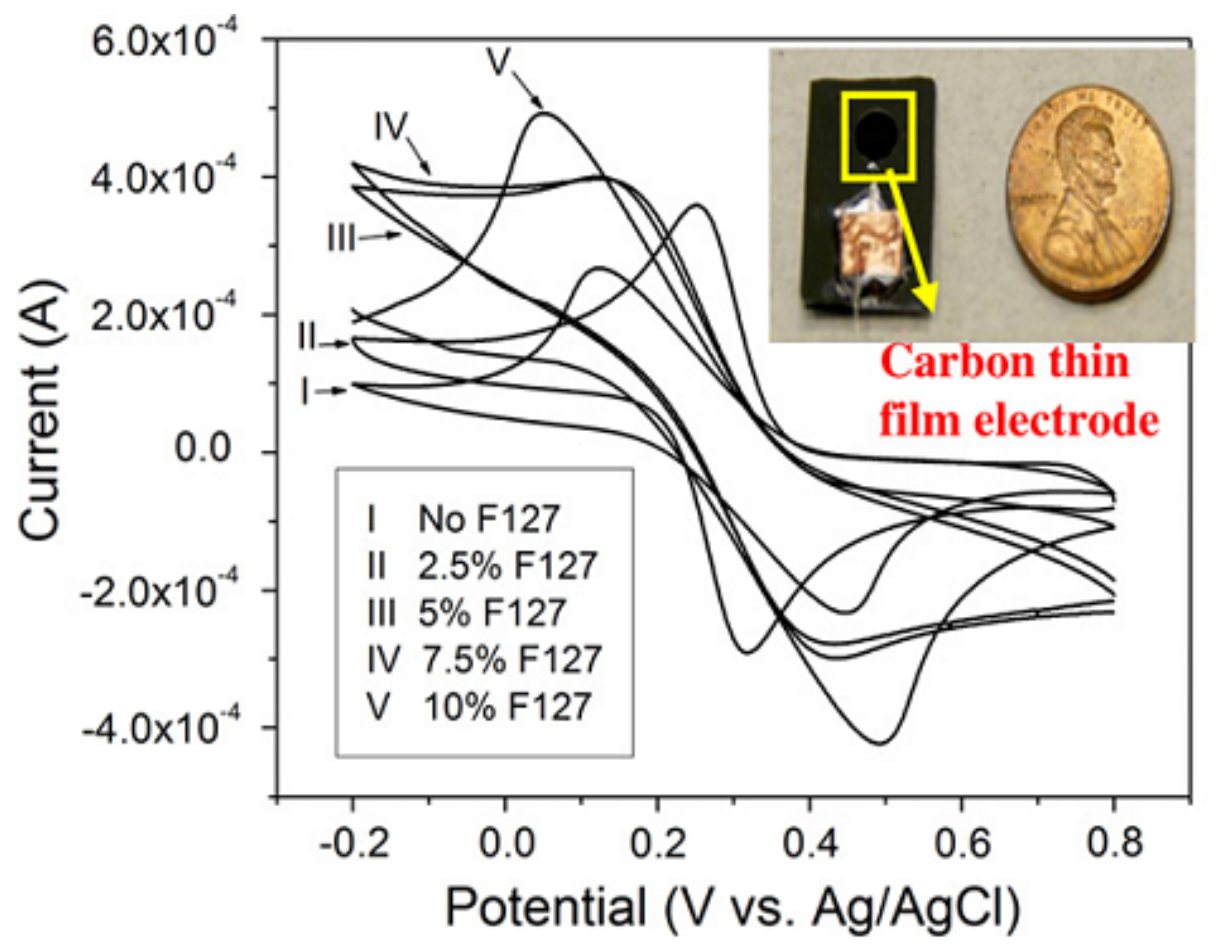

Figure 3.6. Cyclic voltammograms of carbon electrodes derived from SU-8 mixed with (a) no $\mathrm{F} 127,2.5 \% \mathrm{~F} 127,5 \% \mathrm{~F} 127,7.5 \% \mathrm{~F} 127$ and $10 \% \mathrm{~F} 127$ in a $10 \mathrm{mM} \mathrm{K}_{3} \mathrm{Fe}(\mathrm{CN})_{6}+3 \mathrm{M} \mathrm{KCl}$ solution. 
The fraction of the effective surface areas (Aeff) for the electrode with different porogen concentrations measured from the Randles slopes is shown in Figure 3.7. From the plot it can be noticed that the effective surface area (Aeff) increased correspondingly with F127 concentration in the polymer precursor. The porous carbon thin film electrode with $10 \% \mathrm{~F} 127$ concentration had $\mathrm{A}_{\text {eff }} 1.85$ times larger than that of the carbon film obtained from pure SU-8 with the same projected areas. Therefore, the increase in the surface area is expected to be larger when three-dimensional carbon micropillar arrays are fabricated. It is worth mentioning that, by using electrochemical BET, the calculated surface area is mainly from the surface which contributes to the redox reaction through charge-transfer. So, in anisotropic materials (such as graphene) where the charge-transfer efficiency is different on the basal plane and the edge plane, the calculated surface area of the carbon and the total surface area of the carbon might not always be equal. However, it has been previously reported that pyrolytic photoresist carbon which displays electrochemical behavior similar to glassy carbon [22] is more structurally isotropic [23]. Therefore, the surface area results obtained from the electrochemical BET in this work could provide useful information on characterizing surface areas of the carbon microstructures. To explore the maximum possible concentration of F127 in the mixture solution, concentrations more 10\% w/w F127 in SU-8 photoresist were investigated. But, it was difficult to obtain reproducible results since an occasional photoresist film peel off from the substrate was experienced when placed in the water bath. Also, it was observed that as the concentration of F127 increased above 15\%, it formed a thicker white film on the surface of the precursor making it impossible to obtain a uniform layer by spin coating procedure. 


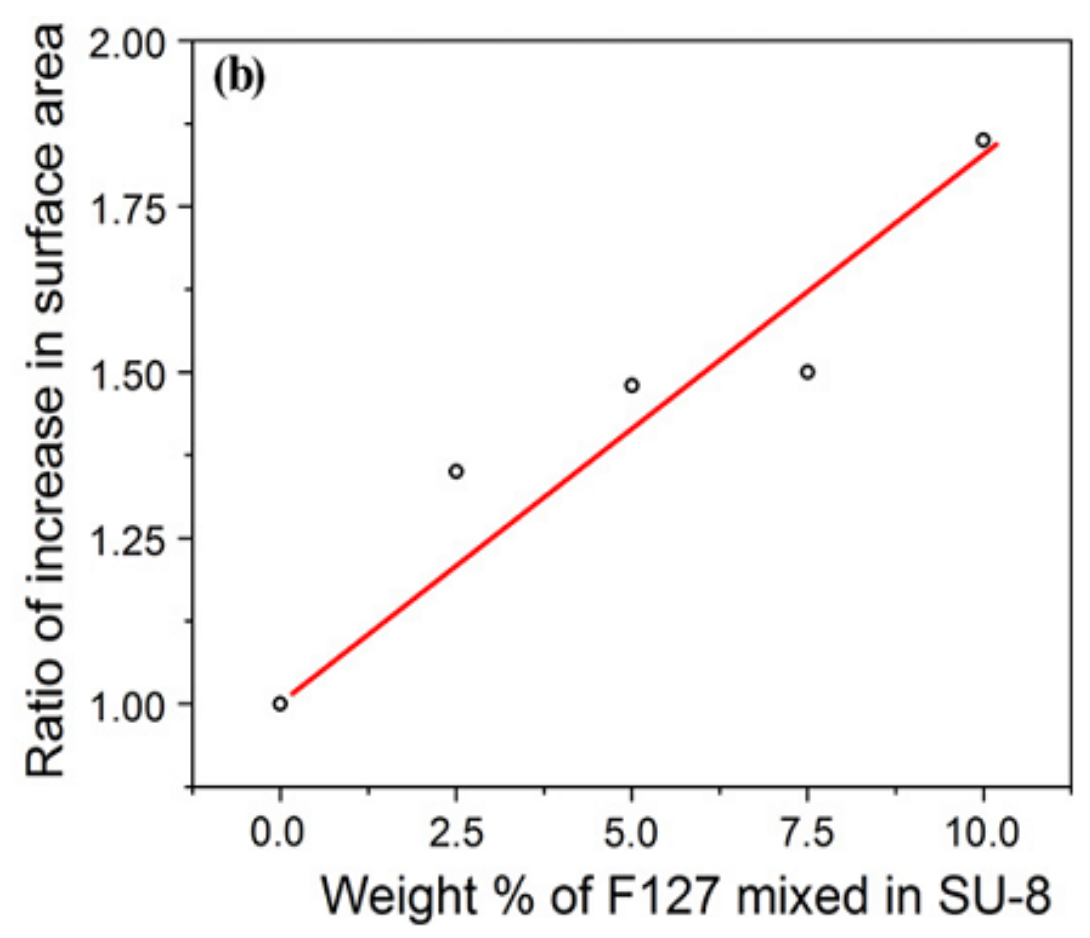

Figure 3.7. Schematic showing the ratio of increase in the surface area according to the different F127 (\% w/w) concentrations.

\subsubsection{Surface Characterization}

The surface morphology of the porous carbon films was further investigated using an AFM in tapping mode as shown in Figure 3.8. AFM images along with roughness profiles for the $10 \%$ F127 + SU-8 samples before and after carbonization are shown in Figure 3.8(a) and (b). The images indicate a uniform mesoporous texture along with pronounced increase in the roughness at the sample surface can be found after carbonization. In contrast, the AFM images (not shown here) for carbon film derived from photoresist precursor with small F127 concentration shows that the surface has no distinguishable porosity except for big roughness, which can be ascribed to the insufficient F127 available to form a thin coating on the whole surface. It is important to 
note that due to the technical limitations of the AFM, it might not be able to reach the real depth of the small pore.
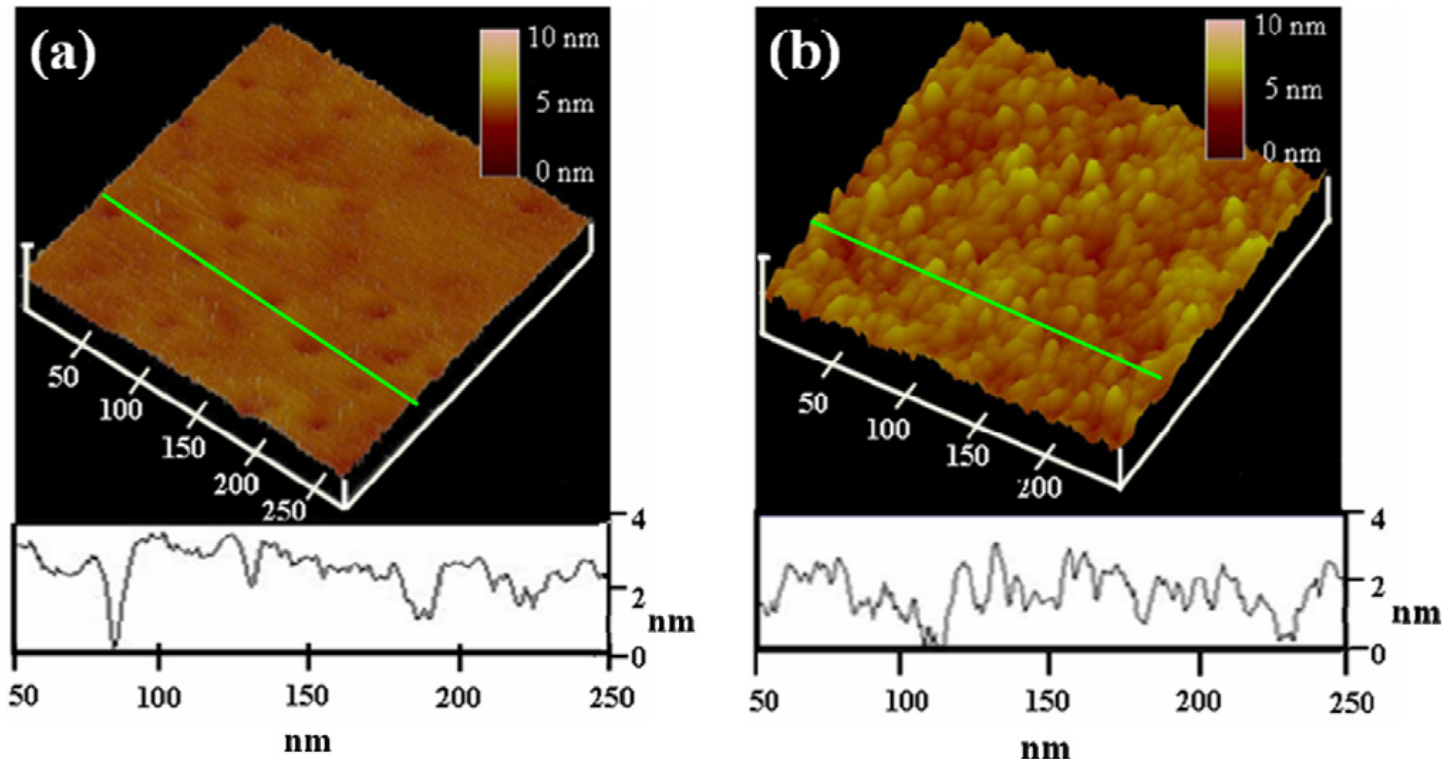

Figure 3.8. AFM image of thin film derived from 10\% F127 mixed photoresist (a) before and (b) after carbonization.

\subsubsection{Using Oxygen RIE Treatment}

\subsubsection{Surface Morphology Characterization}

SEM imaging was employed to investigate the surface of the $3 \mathrm{D}$ carbon micropillars treated by oxygen RIE. Results (Figure 3.9-3.12) indicated rough, jagged carbon surface after the RIE treatment which can be explained by the fact that the free radical oxygen plasma ions start to etch carbon surface. Eventually microporosity is formed due to increased etching at the place of defects on the surface.

The change in the morphology of the carbon surface with RIE time was studied and it was observed that as the etching time increased there was a corresponding increase in the surface roughness shown in Figure 3.9 and 3.10. 


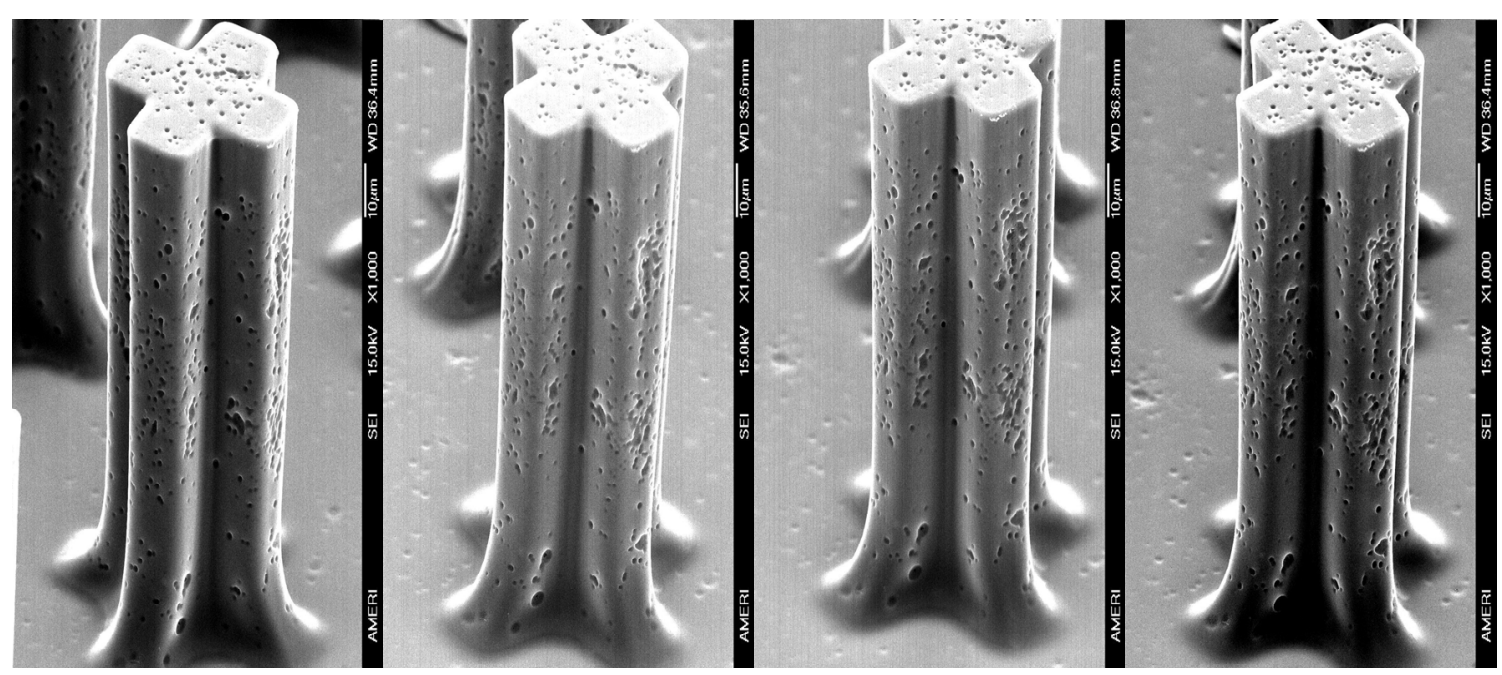

Figure 3.9. Typical SEM images of carbon micropillars etched at pressure 400mTorr, power 100 W, gas flow $100 \mathrm{sccm}$ for (a)20sec, (b) 40sec, (c) 60sec, and (d) $80 \mathrm{sec}$.

Investigation of higher magnification SEM images (Figure $3.10 \mathrm{a}$ and $\mathrm{b}$ ) shows that as the etching time was increased, hierarchical porosity started to form along with an increase in the surface roughness inside the already formed micropores. We already know that pyrolyzed carbon is an isotropic material with both graphitic and amorphous phases. During the RIE treatment, significant etching takes place at the graphic planes compared to the amorphous phase due to the higher etching rate of graphitic phase. The preferential etching at the site of already existing pores can be explained by schematic shown in Figure 3.10c. 


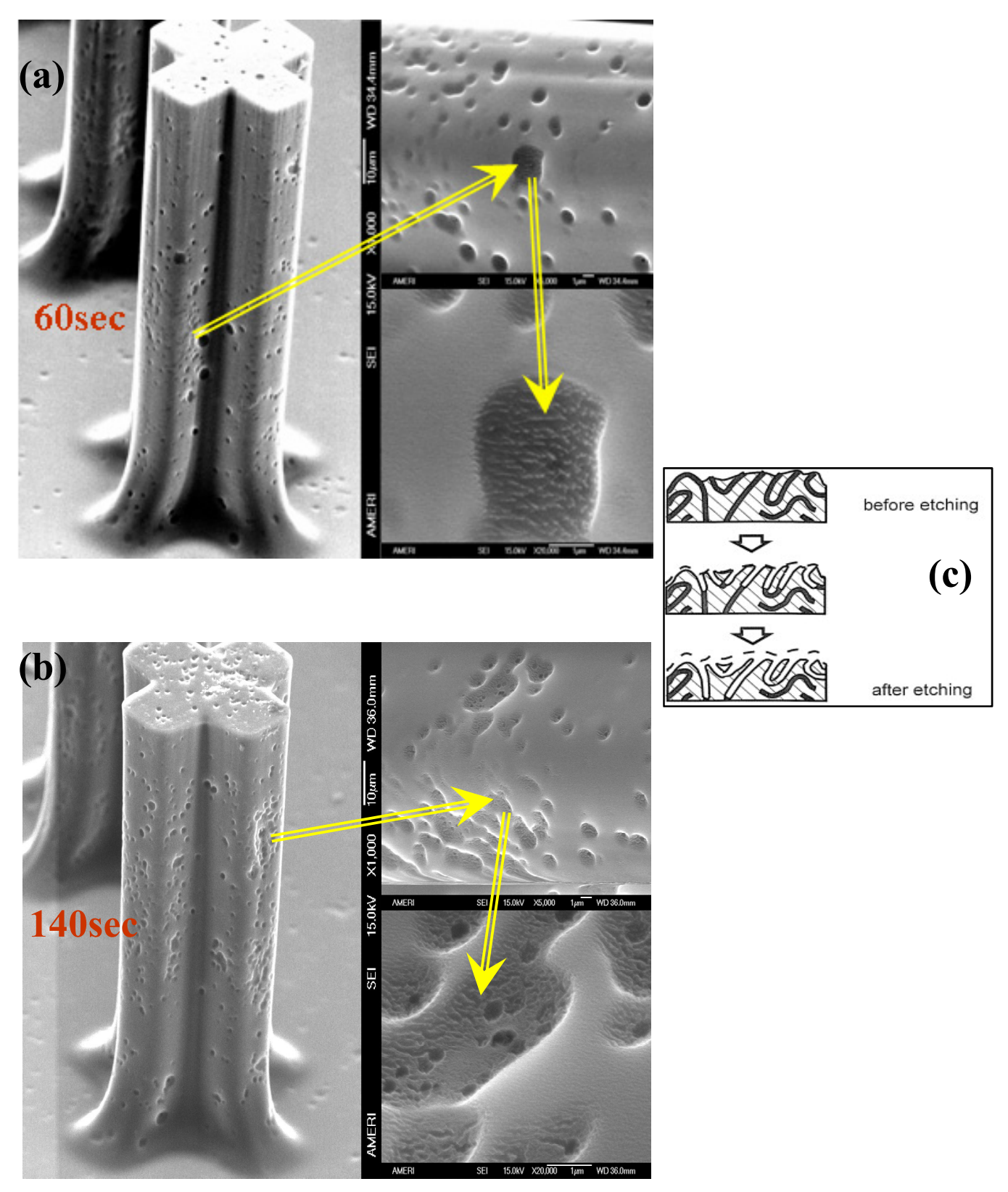

Figure 3.10. High magnification SEM images of carbon surface treated for (a) 60sec, (b) 140sec @ 100 mTorr pressure, 150W power, 10sccm gas flow

Furthermore, by changing the processing parameters such as chamber pressure, power and gas flow in the RIE process, changes in the surface morphology of the carbon micropillars were indistinguishable although surface microporosity was confirmed as in the other cases (Figures 3.11). 


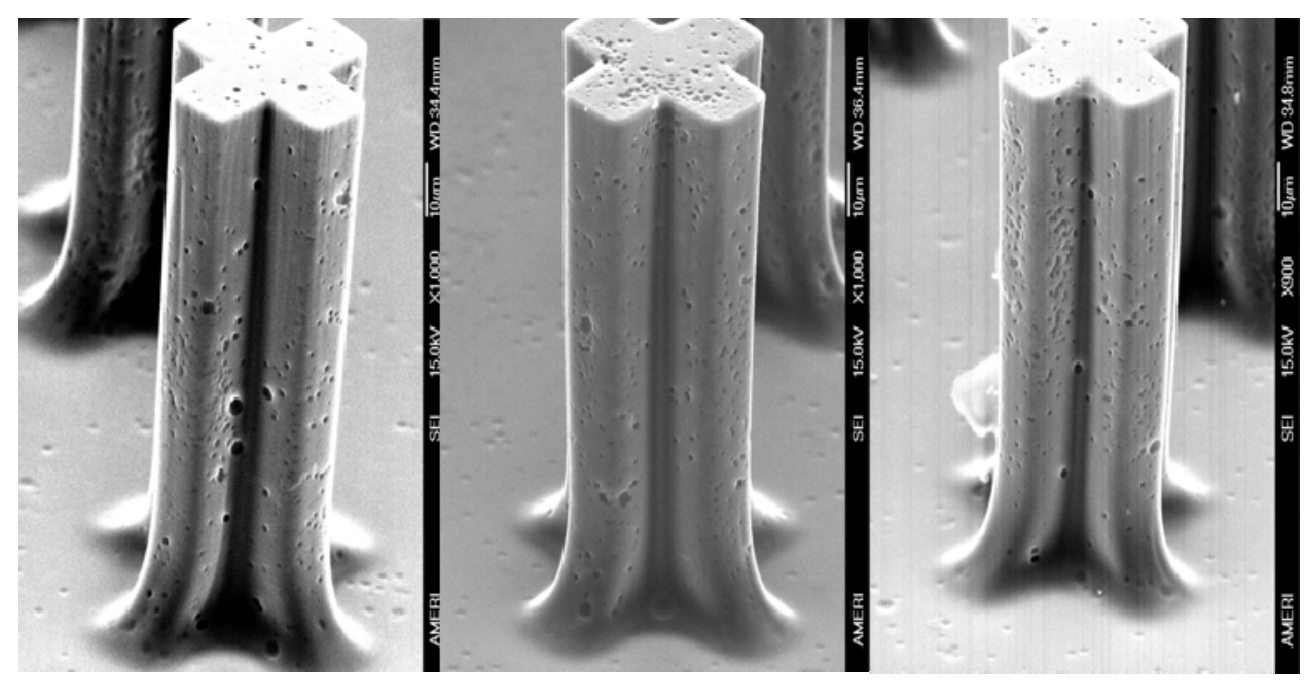

Figure 3.11. Typical SEM images of carbon micropillars etched at (a) pressure-400mTorr, power$150 \mathrm{~W}$, gas flow 10sccm, (b) pressure 100mTorr, power $100 \mathrm{~W}$, gas flow $8 \mathrm{sccm}$, (c) pressure100mTorr, power-150W, gas flow $8 \mathrm{sccm}$ for $60 \mathrm{sec}$.

\subsection{Conclusions}

Two strategies for preparing porous carbon micropillars using F127 as porogen and oxygen RIE treatment have been demonstrated. Surface investigation of the carbon micropillars prepared using F127 as a porogen showed a mesoporous surface texture. It was demonstrated that by increasing the porogen concentration in the photoresist precursor there was a corresponding increase in the surface area of the electrodes. Alternatively, in the case of oxygen RIE treatment, surface roughness and porosity on the carbon surface increased correspondingly with RIE treatment time. Hierarchical porosity was observed at higher etching times due to the preference of the oxygen free radicals to etch at the place of defects. Changing the other processing conditions other than RIE time did not show significant change in the surface morphology of the carbon micropillars. The flexibility to tailor the total surface area of the carbon microstructures makes it a promising process for future C-MEMS applications. 


\subsection{References}

[1] D.B. Burckel, C.M. Washburn, A.K. Raub, S.R.J. Brueck, D.R. Wheeler, S.M. Brozik, R. Polsky, C60: Lithographically defined porous carbon electrodes. 2009, Small, 5, 2792-2796.

[2] V.G. Cathie, F. Elzbieta, J. Krzysztof, F. Marcin, P. Julien, B. Francois, Electrochemical energy storage in ordered porous carbon materials. 2005, Carbon, 43, 587-589.

[3] V.G. Gavalas, N.A. Chaniotakis, Lactate biosensor based on the adsorption of polyelectrolyte stabilized lactate oxidase into porous conductive carbon. 2001, Microchim Acta, 136, 211-215.

[4] J.-H. Yang, V. Penmatsa, S. Tajima, H. Kawarada, C. Wang, Direct amination on 3-dimensional pyrolyzed carbon micropattern surface for DNA detection.2009, Mater. Lett., 63, 2680-2683.

[5] K. Kinoshita, Carbon: electrochemical and physicochemical properties. 1987, John Wiley \& Sons, New York, ISBN: 0471848026.

[6] J.W. Patrick, Porosity in carbons: characterization and applications. 1995, Edward Arnold, London, ISBN: 0470234547.

[7] Z. Yue, J. Economy, Synthesis of highly mesoporous carbon pellets from carbon black and polymer binder by chemical activation, 2006, Micropor Mesopor Mater, 96, 314-320.

[8] N. Patel, K. Okabe, A. Oya. Designing carbon materials with unique shapes using polymer blending and coating techniques. 2002, Carbon, 40, 315-320.

[9] K. Kosuke, K. Onoe, Y. Tatsuaki. Preparation of activated carbon from phenolic resin by alkali activation with sodium hydroxide. 2004, J Soc Inorg Mater Jpn, 11, $165-170$.

[10] O. Klepel, H. Straß, A. Garsuch, K. Böhme, Several ways to produce porous carbon monoliths by template assisted routes, 2007, Mater. Lett., 61, 2037-2039.

[11] Y. Gogotsi, A. Nikitin, H. Ye, W. Zhou, J.E. Fischer, B. Yi, Nanoporous carbidederived carbon with tuneable pore size, 2003, Nat. Mat., 2, 591-594.

[12] C. Wang, G. Jia, L. Taherabadi, M. Madou, A novel method for the fabrication of high aspect ratio C-MEMS structures, 2005, IEEE J Microelectromech Syst, 14, 348-358. 
[13] C. Wang, R. Zaouk, M. Madou, Local CVD of carbon nanofibers from photoresist. 2006, Carbon, 44, 3073-3077.

[14] G.B. Dutt, How critical micelle temperature influences rotational diffusion of hydrophobic probes solubilized in aqueous triblock copolymer solutions. 2005, J Phys Chem B, 109, 4923-4928.

[15] J. Hammacher, A. Fuelle, J. Flaemig, J. Saupe, B. Loechel, J. Grimm, Stress engineering and mechanical properties of SU-8-layers for mechanical applications. 2008, Microsyst Technol., 14, 1515-1523.

[16] Y.Z. Luo, C.V. Nicholas, D. Attwood, J.H. Collett, C. Price, C. Booth, Blockcopoly(oxyethylene/oxybutylene/oxyethylene), E40B15E40, in aqueous solution: micellisation, gelation and drug release. 1993, J. Chem. Soc. Faraday. Trans., 89, 539-546.

[17] D. Rodriguezponce, K. Lozano, T. Eubanks, H. Ahmad, D. Ferrer, Y. Lin, Thermophysical analysis of SU8-modified microstructures created by visible light lithography. 2010, J. Polym. Sci. B: Polym. Phys., 48, 47-54.

[18] O.J.A. Schueller, S.T. Brittain, G.M. Whitesides, Fabrication of glassy carbon microstructures by soft lithography. 1999, 72, 125-139.

[19] J.A. Lee, S.W. Lee, K.C. Lee, S.I. Park, S.S. Lee, Fabrication and characterization of freestanding $3 D$ carbon microstructures using multi-exposures and resist pyrolysis. 2008, J. Micromech. Microeng., 18, 035012-1-035012-10.

[20] R. Harikisun, M. Bertoz, G. Evans, K. Desilvestro, Analysis of porous nanostructures by an 'Electrochemical BET' Method. 2006, International Conference on Nanoscience and Nanotechnology, ICONN'06.

[21] N.G.Patel, A. Erlenkotter, K. Cammann, C. Chemnitius, Fabrication and characterization of disposable type lactate oxidase sensors for dairy products and clinical analysis. 2000, Sensor Actuat. B, 67, 134-141.

[22] O.J.A. Schueller, S.T. Brittain, G.M. Whitesides, Fabrication of glassy carbon microstructures by soft lithography. 1999, Sensor. Actuat. A, 72, 125-139.

[23] J. Kim, X. Song, K. Kinoshita, M. Madou, R. White, Electrochemical studies of carbon films from pyrolyzed photoresist. 1998, J. Electrochem. Soc., 2314-2319. 


\section{CHAPTER 4}

\section{D GRAPHENE COATED CARBON MICROPILLAR ARRAYS FOR ENHANCED ELECTROCHEMICAL SENSING}

\subsection{Introduction}

The high surface area and good electrical conductivity of the $3 \mathrm{D}$ carbon micropillar arrays makes them an ideal platform to integrate variety of nanomaterials to further enhance the performance of C-MEMS based functional devices. Previous studies in our group have shown a 19 fold increase in the specific capacitance for 3D microstructures arrays integrated with carbon nanotubes. In this study, graphene was chosen for integration onto $3 \mathrm{D}$ carbon microarrays due to its high surface area and distinctive electrochemical properties

Graphene is a monolayer of $\mathrm{sp}^{2}$ bonded carbon atoms packed into a honeycomb lattice with distinctive band structure and fascinating physical properties $[1,2]$. The unique physiochemical properties of graphene such as exceptionally low intrinsic electrical resistivity, high surface area, and good mechanical properties make it an attractive material for electrochemical applications such as energy storage [3-5], nanoelectronics [6], mechanical actuators [7], biosensors [8] and filler for nanocomposites [9]. Recent progress in the development of colloidal suspensions of reduced graphene oxide has drawn high interest as an effective precursor for high-volume production of scalable graphene. Essentially graphene films used in electrochemical applications have been prepared by self-assembly [14-16], langmuir-blodgett (LB) assembly [17], vacuum filtration at a liquid-air interface [18], spray coating [19], dipcoating [13], electrophoretic deposition [20] and spincoating [21]. While the 
aforementioned methods can be used to prepare graphene films on large area substrates, it is a challenge to integrate graphene onto patterned three-dimensional (3D) structures. Thus, it is of interest to develop new facile approaches to integrate graphene onto the $3 \mathrm{D}$ structures to take advantage of potential merits such as very large surface areas and enhanced chemical functionality. For example, three-dimensional battery architectures have shown a $350 \%$ larger energy capacity compared to traditional two-dimensional designs in the same areal footprint [22].

The electrostatic spray deposition (ESD) method is a versatile technique that has been successfully applied for the deposition of carbon nanotubes, carbon nanospheres and different metal oxide materials for electrochemical applications [23-27]. Compared to other techniques which are capable of coating 3D microstructures such as atomic layer deposition (ALD) [28], electrodeposition [29,30], and modified spincoating [31], ESD is appealing due to its high deposition rate, good uniformity and ability to deposit wide variety of functional materials. In the ESD technique, the precursor solution is atomized into an aerosol and precisely directed onto a heated substrate by high electric potential applied between the spray nozzle and the substrate. Droplets produced by electrospraying are highly charged, that prevents their coagulation and promotes self-dispersion [32]. The morphology of the sprayed films can be controlled by adjusting deposition parameters such as flow rate, applied potential, nozzle geometry, substrate temperature, and precursor solution composition [33]. Recently, our group reported the uniform integration of carbon nanotubes (CNTs) on the surface of high aspect ratio 3D carbon microstructures by depositing catalyst particles using the ESD [24]. In addition, by tailoring the viscosity of the precursor solution, polymeric and carbon nanowires with 
predefined lengths have been deposited onto 3D carbon micropillar arrays with great reproducibility [34-36]. However, the conformal coating of graphene on 3D complex microstructures has not been reported.

In this chapter, a novel approach to fabricate 3D graphene nanosheets encrusted carbon micropillars using combined top-down [photo-lithography] and bottom-up processes [electrostatic spray deposition] is presented. The effect of critical ESD parameters such as deposition time, substrate temperature and substrate to nozzle distance on the substrate morphology was investigated. By comparing the electrochemical performance of 3D graphene/carbon micropillar electrode array to bare 3D carbon micropillar electrode array, an apparent increase in the effective surface area and faster charge transfer was noticed along with a linear response for wide range of hydrogen peroxide detection. This methodology can be extended to conformally coat different functional nanomaterials onto high aspect ratio microstructures for wafer-level processing.

\subsection{Materials and Methods}

Reduced graphene nanosheets used in this work were obtained from Cheaptubes Inc, USA. 1,2 propanediol was purchased from Sigma Aldrich, USA. NANO ${ }^{\text {TM }}$ SU-8 and $\mathrm{NANO}^{\mathrm{TM}}$ developer was purchased from Microchem, USA.

\subsubsection{Fabrication Process}

Briefly, a $200 \mu \mathrm{m}$ thick NANO ${ }^{\mathrm{TM}}$ SU-8 100 photoresist film is spincoated onto silicon oxide (4", (100)-oriented, n-type) wafer at $500 \mathrm{rpm}$ for $12 \mathrm{sec}$ then $3000 \mathrm{rpm}$ for $30 \mathrm{sec}$. The photoresist film was soft baked at $65^{\circ} \mathrm{C}$ for $10 \mathrm{~min}$ followed by hard bake for 30 min at $95{ }^{\circ} \mathrm{C}$ in a box oven. The photoresist is patterned by a broadband mercury lamp 
exposure for $105 \mathrm{sec}$ using an OAI contact aligner (light intensity, $12 \mathrm{~mW} / \mathrm{cm}^{2}$ ). Post expose bake was carried out at $65{ }^{\circ} \mathrm{C}$ for $1 \mathrm{~min}$ and $95{ }^{\circ} \mathrm{C}$ for $3 \mathrm{~min}$. The UV exposed samples were developed using NANO ${ }^{\mathrm{TM}} \mathrm{SU}-8$ developer for $10 \mathrm{~min}$ followed by IPA rinse and $\mathrm{N}_{2}$ drying. The whole carbonization process was conducted in a Lindberg alumina-tube furnace with a constant flow of $500 \mathrm{sccm}$ forming gas $\left(95 \% \mathrm{~N}_{2}+5 \% \mathrm{H}_{2}\right)$. Previous research has shown that the annealing temperature and the gas used during the pyrolysis process play a critical role in the microstructure of the final product [42]. In this work, 3D carbon micropillars were obtained by heating polymer micropillars from room temperature to $350{ }^{\circ} \mathrm{C}$ at $5{ }^{\circ} \mathrm{C} / \mathrm{min}$ with $40 \mathrm{~min}$ hold time and then ramping to $1000{ }^{\circ} \mathrm{C}$ at $5{ }^{\circ} \mathrm{C} / \mathrm{min}$ for $60 \mathrm{~min}$ before being cooled down to room temperature.

\subsubsection{Electrostatic Spray Deposition (ESD) Process}

The details of the ESD process used in this work have been reported previously $[25,26]$. A photograph of the ESD setup used in our lab is shown in Figure 4.1. The precursor solution was prepared by homogenizing $0.3 \mathrm{mg}$ graphene nanosheets in $0.3 \mathrm{ml}$ 1,2 propanediol using a high power ultrasonic dispenser (Sonics VC750) for $30 \mathrm{~min}$. The resultant solution was pumped through a needle at a flow rate of $1 \mathrm{ml} / \mathrm{h}$ onto $3 \mathrm{D}$ carbon micropillars. Samples were deposited at three different substrate temperatures of $250{ }^{\circ} \mathrm{C}$, $300{ }^{\circ} \mathrm{C}$, and $400{ }^{\circ} \mathrm{C}$ and at $10 \mathrm{~min}, 30 \mathrm{~min}$, and $60 \mathrm{~min}$ deposition time. The three different distances between the nozzle and the substrate were set at $3 \mathrm{~cm}, 5 \mathrm{~cm}$, and $8 \mathrm{~cm}$ with a voltage of $\sim 5-6 \mathrm{kV}$. The whole deposition process was carried out inside a fume hood. 


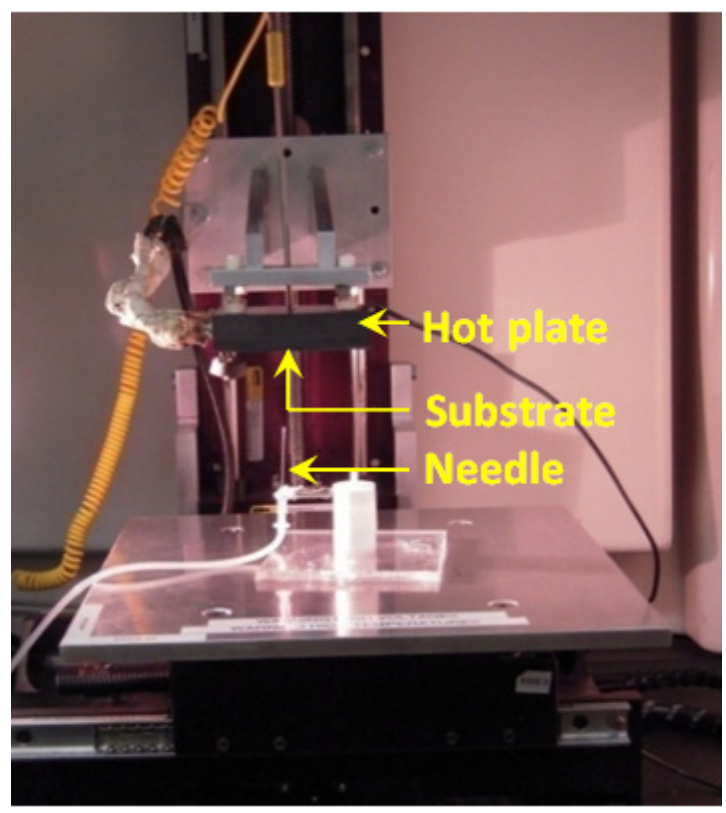

Figure 4.1. Photograph of the actual ESD setup in our lab.

\subsubsection{Characterization}

The morphology of the graphene/carbon microstructures was investigated using JOEL 6335 FE- SEM scanning electron microscopy. Transmission electron microscopy (TEM) analysis of graphene was performed using a TECNAI-F20 FEG TEM facility. Raman spectroscopy measurements were carried out with an argon ion laser system (Spectra Physics, model 177G02) of $\lambda=514.5 \mathrm{~nm}$ at a laser power of ca. $7 \mathrm{~mW}$. The chemical composition of graphene before and after deposition was investigated by an Ulvac $\Phi 3300$ x-ray photoelectron spectroscopy (XPS) with an anode source providing Al $\mathrm{K} \alpha$ radiation. The electron takeoff angle was $45 \pm 3^{\circ}$ relative to the substrate surface. For the hydrogen peroxide detection, electrochemical measurements were performed using a CHI 660C workstation with a N2 flowed typical three-electrode cell at room temperature. The graphene/carbon micropillars sample was used as working electrode, a platinum wire was used as the counter electrode and all potentials were measured against 
an $\mathrm{Ag} / \mathrm{AgCl}$ reference electrode. To make the working electrode, an electrical contact was established by using a copper wire and copper tape. The undesired active electrode surface was masked using epoxy.

\subsection{Results and Discussion}

\subsubsection{Fabrication and Characterization}

The simple methodology for preparing graphene encrusted 3D micropillars is schematically shown in Figure 4.2: The fabrication process begins by patterning a thick negative-tone photoresist, SU-8, into 3D micropillars by traditional photolithography. Next, the polymer micropillar arrays are pyrolyzed to outgass the volatile components in the organic photoresist leaving behind solid carbon. By just changing the processing conditions during photolithography, carbon microstructures with different shapes and aspect ratios can be fabricated. The large surface area of the 3D carbon micropillars makes them a favorable platform for both electrochemical sensing and to integrate graphene. Finally, the conformal coating of graphene onto the carbon microstructures is carried out by the ESD technique. 
(a)

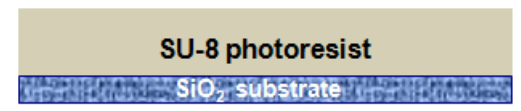

(b)

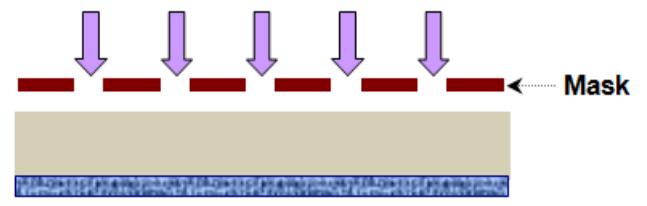

(c)

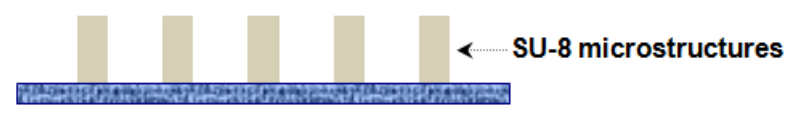

(d)

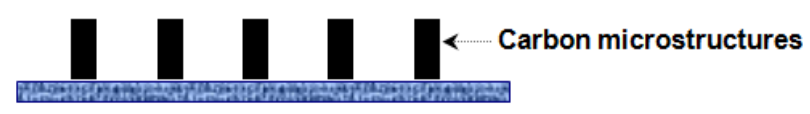

(e)

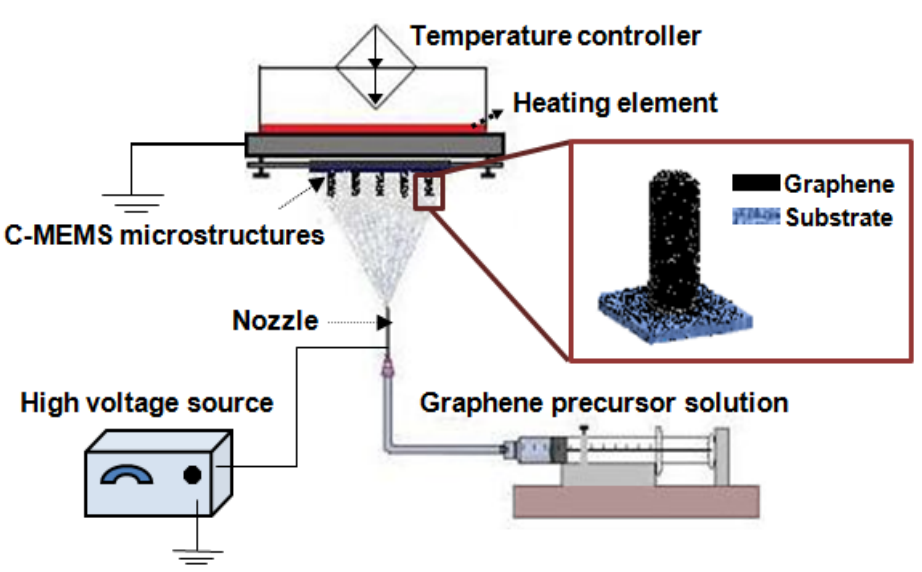

Figure 4.2. Schematic showing a-d) the fabrication of 3D carbon micropillars, and e) deposition of graphene on carbon micropillars using ESD technique.

The morphology of the graphene nanosheets after deposition was characterized by electron microscopy. Figure 4.3a shows the SEM image of pristine graphene nanosheets deposited on the substrate. The image suggests that nanosheets are small stacks of graphene layers with size varying between 2-5 $\mu \mathrm{m}$. As expected, the graphene nanosheets maintained their fidelity even after deposition. The high resolution transmission electron microscopy image of the graphene nanosheets (Figure $4.3 \mathrm{~b}$ ) clearly indicates that it is made up of domains with an interplanar spacing of $\sim 0.33 \mathrm{~nm}$ and size $\sim 5 \mathrm{~nm}$. Further, 
Raman spectra was investigated (Figure 4.3c) to distinguish the ordered and disordered crystal structures of carbon. A prominent $\mathrm{G}$ band at $\sim 1580 \mathrm{~cm}^{-1}$ that corresponds to the breathing mode of $\kappa$-point phonons of $A_{1 g}$ symmetry and a broad $\mathrm{D}$ band at $\sim 1350 \mathrm{~cm}^{-1}$ due to first-order scattering of the $\mathrm{E}_{2 \mathrm{~g}}$ phonons were observed [43]. The quality of graphene was evaluated by calculating the $\mathrm{I}_{\mathrm{D}} / \mathrm{I}_{\mathrm{G}}$ ratio from the Raman spectra. Relatively low value of $\sim 0.26$ suggests a low defect density in the crystal structure of graphene nanosheets. By using the empirical Tuinstra-Koenig relation [44] that relates the $\mathrm{I}_{\mathrm{D}} / \mathrm{I}_{\mathrm{G}}$ ratio to the crystallite size of graphitic samples, it can be concluded that the graphene is comprised of ordered $\mathrm{sp}^{2}$ domains with an average size of $\sim 4 \mathrm{~nm}$. The domain size is in good agreement with the size determined by the $\mathrm{I}_{\mathrm{D}} / \mathrm{I}_{\mathrm{G}}$ ratio.
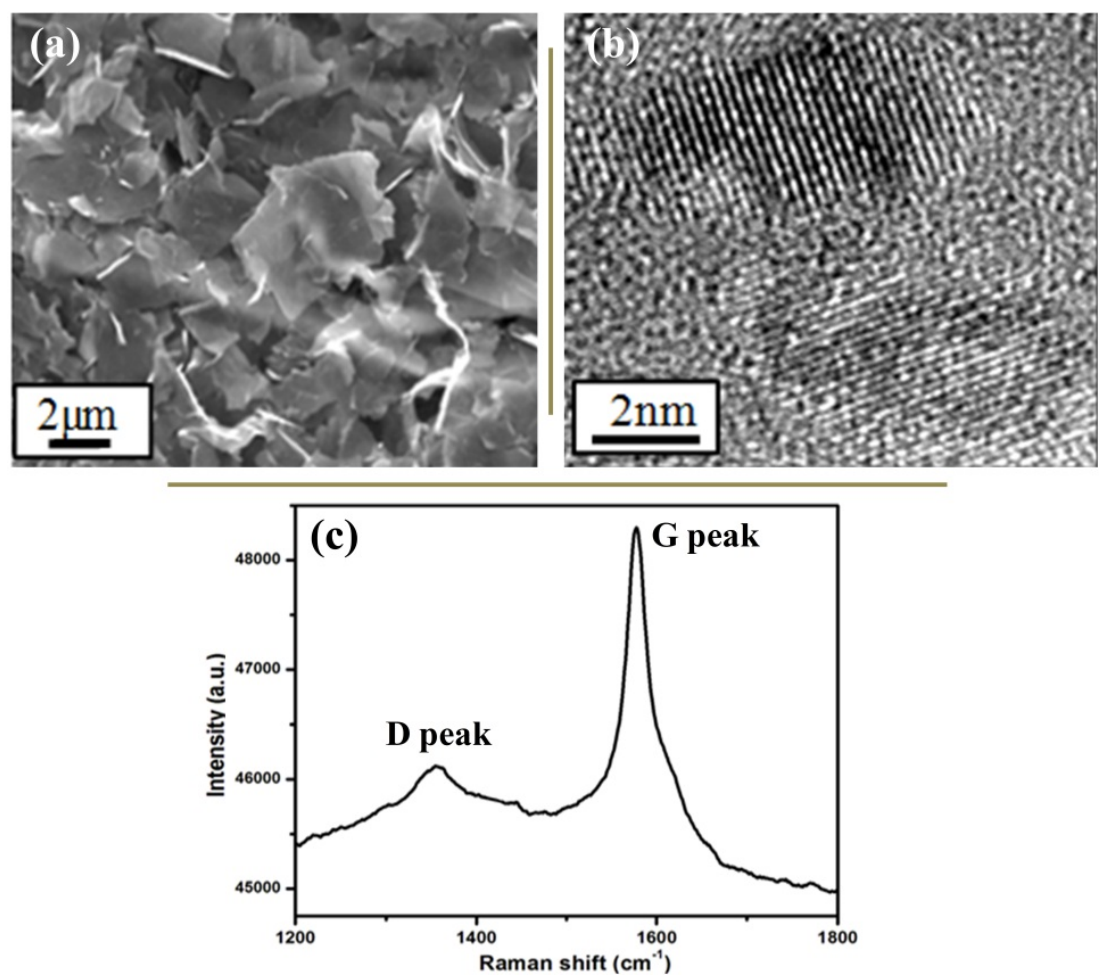

Figure 4.3. a) Typical SEM image of pristine graphene deposited on silicon oxide, b) HRTEM image of graphene nanosheets, and c) Raman spectra (G and D peaks) of as-deposited graphene nanosheets. 


\subsubsection{Effect of ESD Processing Conditions}

In order to investigate the relationship between the graphene film morphology and the ESD deposition parameters, we varied the substrate temperature, deposition time and nozzle to substrate distance during graphene deposition. As seen in Figure 4.4, there is a distinct variation in the graphene film morphology on the substrate by changing the deposition conditions. Initially, the deposition time was fixed at $30 \mathrm{~min}$ and the temperature was varied between $200-400{ }^{\circ} \mathrm{C}$. At $200{ }^{\circ} \mathrm{C}$ deposition temperature, non uniform distribution of graphene islands was observed on the substrate (Figure 4.4a). But as the substrate temperature was increased to $300{ }^{\circ} \mathrm{C}$, the SEM (Figure 4.4b) image clearly shows porous reticular structured film with a feature size of $\sim 10-20 \mu \mathrm{m}$. Further when the temperature was increased to $400{ }^{\circ} \mathrm{C}$, no apparent coating of graphene was observed on the substrate (Figure 4.4c) which could be a result of the oxygen present in the air reacting with the carbon in graphene and eventually outgas as carbon dioxide at high temperature. Secondly, the deposition time was varied between $10 \mathrm{~min}, 30 \mathrm{~min}$ and 60 min keeping the deposition temperature constant at $200{ }^{\circ} \mathrm{C}$ and 3 nozzle to substrate distance (NSD). As expected the graphene film grew denser with an increase in the deposition time (data not shown). 10min deposition of the precursor was not sufficient for the graphene film to completely cover the substrate and it was apparent that as the deposition increased to $30 \mathrm{~min}$ and $60 \mathrm{~min}$, the coverage of the film on the substrate was more pronounced. Analysis showed that $200{ }^{\circ} \mathrm{C}$ and 60 min deposition time produces porous structured film with graphene "rings" of diameter $\sim 5-10 \mu \mathrm{m}$ (Figure $4.4 \mathrm{~d}$ ). Lastly, the effect of NSD was investigated by fixing the NSD at 3,5 and $8 \mathrm{~cm}$. Results observed from different SEM images showed that $3 \mathrm{~cm}$ distance yielded the best film morphology 
(Figure 4.4d). Upon increasing the distance, the spray is more influenced by a viscous drag force. Also, the trajectory and the flight time of the spray is largely influenced by the columbic charge of the droplets, space-charge forces due to the repulsive interaction between charged droplets and thermophoresis force. As a result, it was observed that in the case of both $5 \mathrm{~cm}$ and $8 \mathrm{~cm}$ NSD, the diameter of the graphene "rings" formed by precipitation and evaporation of solvent, increased with NSD (Figure 4.4 e,f). In the case of $8 \mathrm{~cm} \mathrm{NSD}$, the graphene film coverage was non uniform with the presence of random graphene islands due to the large distance between the nozzle and the substrate.

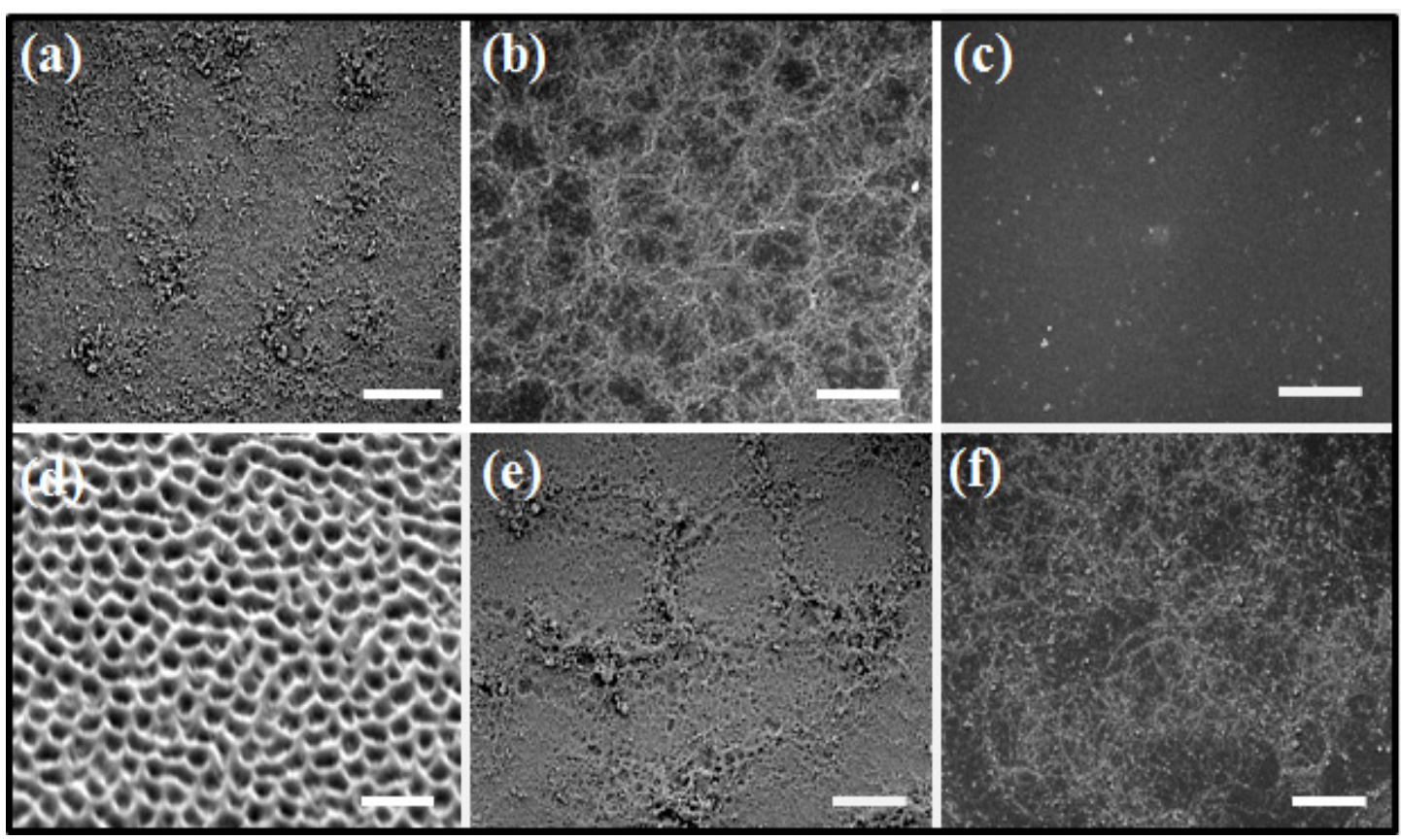

Figure 4.4. Typical SEM images of graphene film deposited on carbon at a) $200{ }^{\circ} \mathrm{C}$, b) $300{ }^{\circ} \mathrm{C}$, c) $400{ }^{\circ} \mathrm{C}$ for $30 \mathrm{~min}, 3 \mathrm{~cm} \mathrm{NSD}$; d) $3 \mathrm{~cm}$, e) $\left.5 \mathrm{~cm}, \mathrm{f}\right) 8 \mathrm{~cm}$ NSD for $60 \mathrm{~min}, 200{ }^{\circ} \mathrm{C}$. Scale bar is $20 \mu \mathrm{M}$.

One of the main advantages of using the ESD technique is the ability to configure the system for seamlessly coating graphene onto both $2 \mathrm{D}$ and high aspect ratio $3 \mathrm{D}$ structures. Figure 4.5 shows the typical SEM images of graphene/ carbon micropillars at 
two different deposition conditions showing interesting film morphologies. Figure 4.5a and $4.5 \mathrm{~b}$ shows the SEM image of carbon micropillars coated with graphene at $200{ }^{\circ} \mathrm{C}$ for $60 \mathrm{~min}$ and $300{ }^{\circ} \mathrm{C}$ for $30 \mathrm{~min}$ deposition time. By carefully observing the image a conformal coating of graphene nanosheets on the surface of the carbon micropillars and the substrate can be noticed (Figure $4.5 \mathrm{~b}$ inset). Due to the fact that the spray was directed at the top of micropillars, the coating of graphene at the top is a little denser relative to the bottom. The porous structure of the film on the substrate could be attributed to the constant precipitation and evaporation of graphene precursor solution. This kind of porous structure is advantageous in electrochemistry since it provides more surface area accessible to the electrolyte. The proposed theory behind the formation of porous morphology is schematically shown in Figure 4.5c. Since the morphology of the film deposited is largely influenced by the processing conditions employed during deposition such as droplet size, decomposition temperature and spreading behavior of the precursor solution on the substrate, it is presumed that when the precursor droplets come in contact with the surface, it tries to discharge by transferring its charge to the grounded substrate. The spreading of this droplet is strongly influenced by spreading coefficients and the wetting angle of the droplet with the substrate, shown in equation 1 [32].

$$
\cos \theta=\frac{\gamma_{s-g}-\gamma_{s-l}}{\gamma_{l-g}}
$$

where $\gamma_{\mathrm{s}-\mathrm{g}}$ denotes the substrate-ambient gas interfacial tension, $\gamma_{\mathrm{s}-1}$ between substrate and droplet liquid, and $\gamma_{1-\mathrm{g}}$ between droplet liquid and ambient gas. 

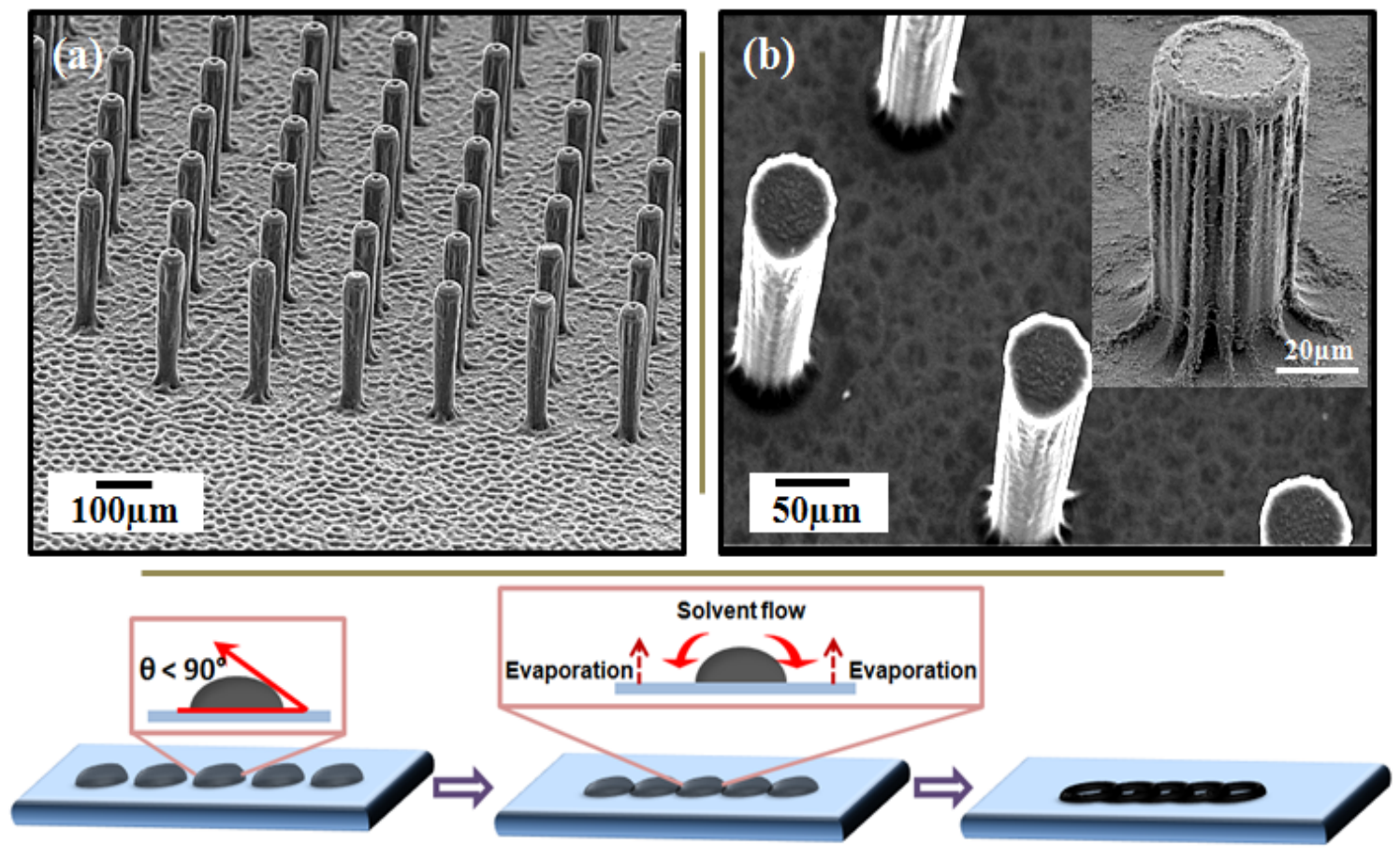

Figure 4.5. Typical SEM images of graphene deposited carbon micropillars at conditions a) $200{ }^{\circ} \mathrm{C}$ for $60 \mathrm{~min}$; b) $300{ }^{\circ} \mathrm{C}$ for $30 \mathrm{~min}$; Inset: graphene coated carbon micropillar c) Schematic showing the proposed methodology explaining the formation of porous film structure.

If the droplet has wetting angle $\theta<90^{\circ}$ it starts to spread on the heated substrate and simultaneously evaporate due to the heat transfer between the substrate and droplet. However, due to slightly higher local temperature at the edge compared to the middle of the droplet, the evaporation at the edges precedes the middle. With the inhomogeneous concentration profile and the temperature gradient, edges, form the favorable sites for nucleation and precipitation of the solute. Further the solution in the middle tends to flow towards the edges and this coupled with evaporation of solvent at edges leads to the formation of solute "rings" on the substrate. Finally, after the solvent has completely evaporated, an interconnected porous structure is formed. 
Recently, a lot of research efforts have been focused on studying the thermal reduction of graphene oxide to graphene at different temperatures $[45,46]$. For graphene oxide, the first major mass transition due to the evaporation of interstitial $\mathrm{H}_{2} \mathrm{O}$ occurs at $110{ }^{\circ} \mathrm{C}$ and the second major exothermic mass loss takes place at $220{ }^{\circ} \mathrm{C}$ [45]. In our case, during the deposition of graphene precursor solution using ESD, the substrate temperature is maintained at $200{ }^{\circ} \mathrm{C}$ or higher to evaporate the residual solvents. In order to evaluate the thermal transition of graphene nanosheets before and after deposition on the silicon oxide substrate, XPS investigation was conducted. The broadscan XPS elemental analysis of the graphene film deposited on $\mathrm{SiO}_{2}$ shows only the presence of carbon and oxygen (Figure 4.6). It is clearly visible that after graphene deposition there is an apparent increase in the carbon peak intensity and a slight decrease in the oxygen peak could be attributed the minor deoxygenation of graphene. The high resolution $\mathrm{C} 1 \mathrm{~s}$ and $\mathrm{O}$ 1s XPS scans of graphene thin film before and after deposition at $250{ }^{\circ} \mathrm{C}$ is shown in (inset Figure 4.6). By using the spectral manifold intensities, the carbon to oxygen ratio $(\mathrm{C} / \mathrm{O})$ of raw graphene nanosheets powder was calculated to be $3.75 \pm 0.2$. The $\mathrm{C} 1 \mathrm{~s}$ region was deconvoluted into $\mathrm{C}-\mathrm{C}$ at $284.6 \mathrm{eV}, \mathrm{C}-\mathrm{O}$ (presumably as $\mathrm{C}-\mathrm{OH}$ (vide infra)) at $286.7 \mathrm{eV}, \mathrm{C}=\mathrm{O}$ at $288.0 \mathrm{eV}$, and $\mathrm{COOH}$ at $289.1 \mathrm{eV}$ [47]. Alternatively, for the ESD deposited graphene nanosheets film a slightly higher $\mathrm{C} / \mathrm{O}$ ratio of $4.23 \pm 0.2$ was observed which could be attributed to thermal reduction at the deposition temperature. 


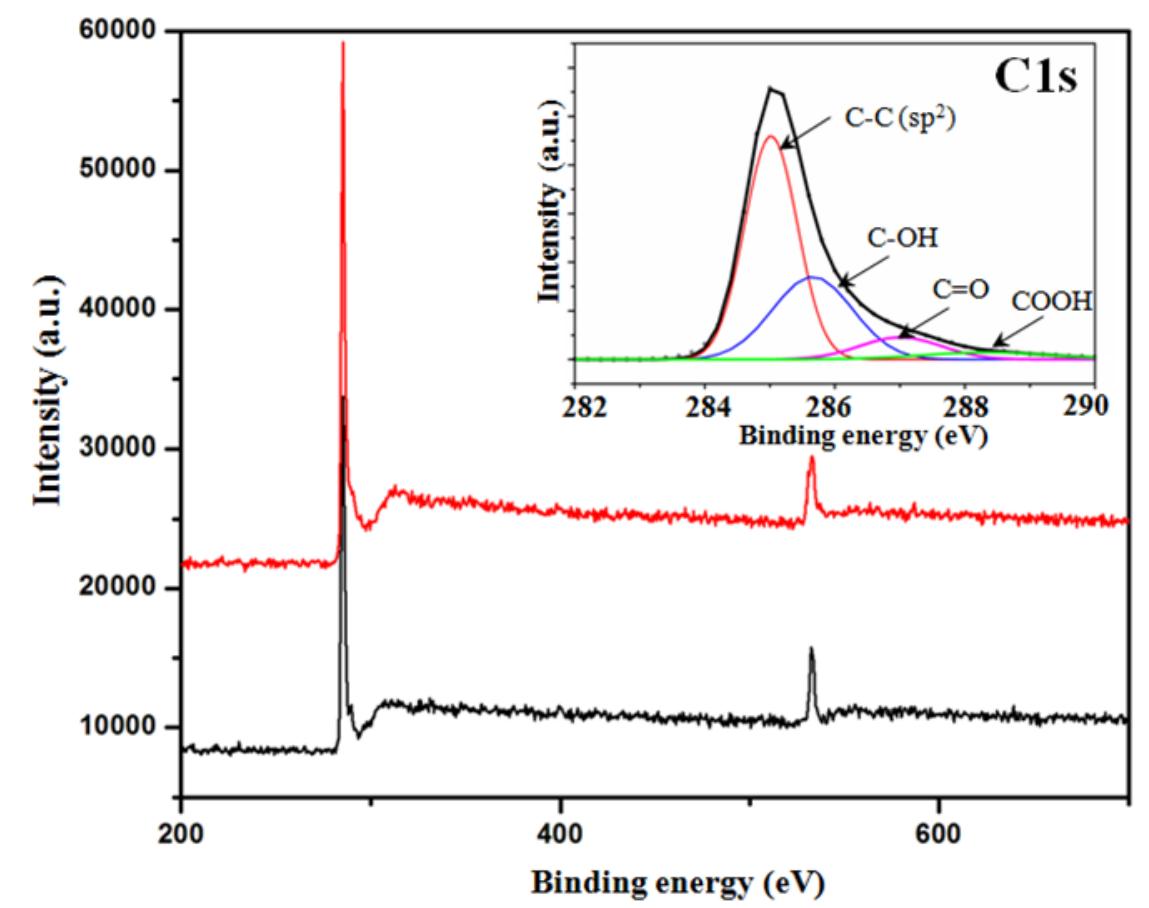

Figure 4.6. Broadscan XPS spectra of graphene before and after deposition; Inset: Deconvoluted high resolution carbon XPS spectrum.

\subsubsection{Hydrogen Peroxide Detection}

In order to demonstrate the feasibility of $3 \mathrm{D}$ graphene/carbon micropillar electrode arrays for electrochemical sensing, a hydrogen peroxide detection system was conducted. Rapid and accurate detection of hydrogen peroxide is of great importance in clinical analyses and in particular for biosensors development [48]. At first, the electrocatalytic activity of graphene/carbon micropillar and bare carbon micropillar electrode arrays were compared by examining the amperometric response of the electrodes towards hydrogen peroxide. Figure 4.7 shows the typical cyclic voltammetry curves in a $1 \mathrm{mM}$ phosphate-buffered saline $(\mathrm{pH}=7.2)$ solution containing $20 \mathrm{mM} \mathrm{H}_{2} \mathrm{O}_{2}$ between a -0.2 to $0.5 \mathrm{~V}$ potential window. As expected, the graphene/carbon micropillar electrode array displays an excellent catalytic activity and facilitates faster electron 
transfer. Comparing the area under the CV curves (Figure 4.7), it is evident that the total charge transfer of the graphene/ carbon micropillar electrode array is also considerably greater. The signal enhancement could be attributed to the increase in electrode catalytic surface and in particular to the high conductivity and better charge transfer of graphene. Note that previous reports have demonstrated that the number of layers in graphene nanosheets has no effect on the electrochemical response towards the electrolyte [49].

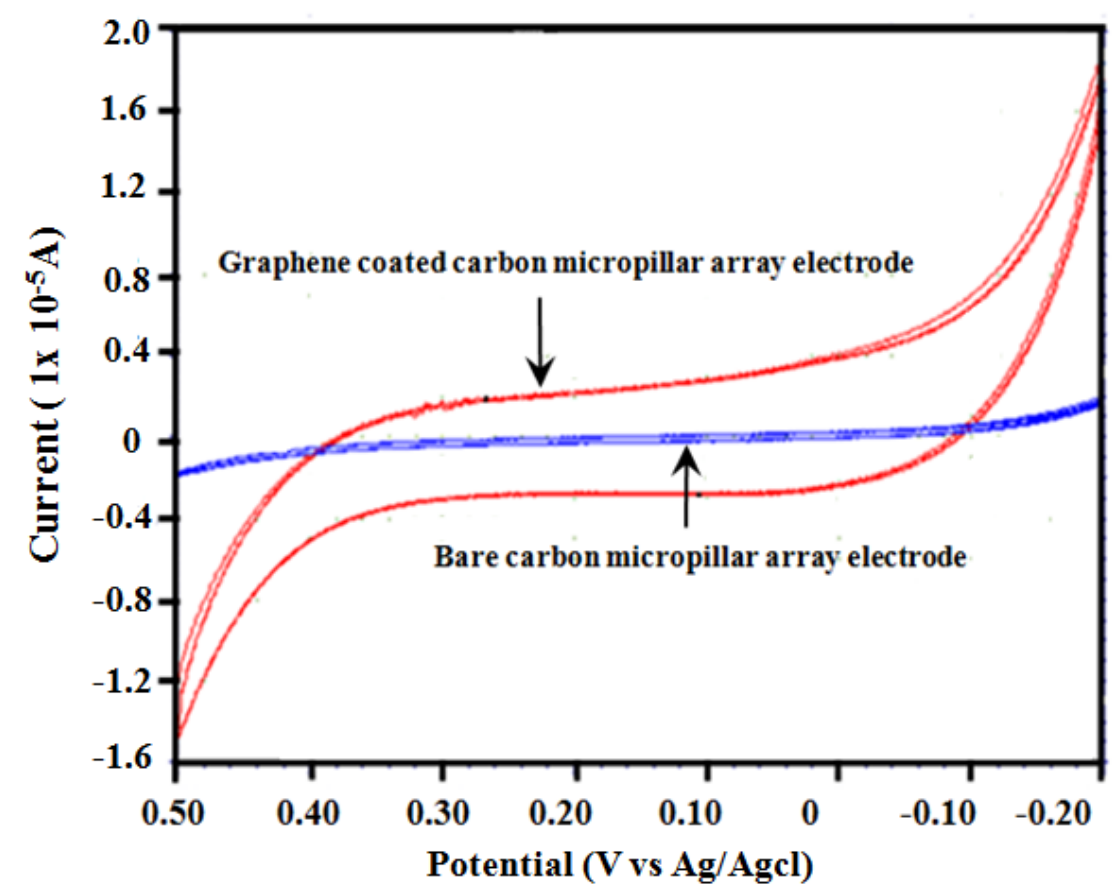

Figure 4.7. Typical cyclic voltammetry curves comparing the amperometric response of bare and graphene-coated carbon micropillar electrode arrays.

Next attention was turned to the response of the graphene modified 3D carbon micropillar electrode array for different concentrations of $\mathrm{H}_{2} \mathrm{O}_{2}$. Figure 4.8 shows the amperometric response of the electrode to the successive additions of $250 \mu \mathrm{M}$ hydrogen peroxide into stirring PBS $(\mathrm{pH}=7.2)$ at a $0.4 \mathrm{~V}$ working potential. The catalytic reduction of $\mathrm{H}_{2} \mathrm{O}_{2}$ at the surface of graphene/carbon micropillar electrode array is very fast in 
reaching a dynamic equilibrium upon each addition of $\mathrm{H}_{2} \mathrm{O}_{2}$, generating a steady-state current signal within $5 \mathrm{sec}$. The modified electrode exhibits a rapid and sensitive response to the change of hydrogen peroxide concentration and an obvious increase in current upon successive addition of $\mathrm{H}_{2} \mathrm{O}_{2}$. The response of the graphene/ carbon micropillar electrode array to $\mathrm{H}_{2} \mathrm{O}_{2}$ is nearly linear up to $5.5 \mathrm{mM}$ with a sensitivity of $0.07 \mu \mathrm{A} / \mu \mathrm{Mcm}^{2}$ and with high saturation. The corresponding calibration plot shown in inset Figure 4.8 indicates the linear increase in response current upon the increase in $\mathrm{H}_{2} \mathrm{O}_{2}$ concentration with a linear range from $250 \mu \mathrm{M}$ to $5.5 \mathrm{mM}$. Based on the electrochemical results, the graphene/carbon micropillar electrode arrays was demonstrated as a promising platform for constructing sensitive electrochemical sensors.

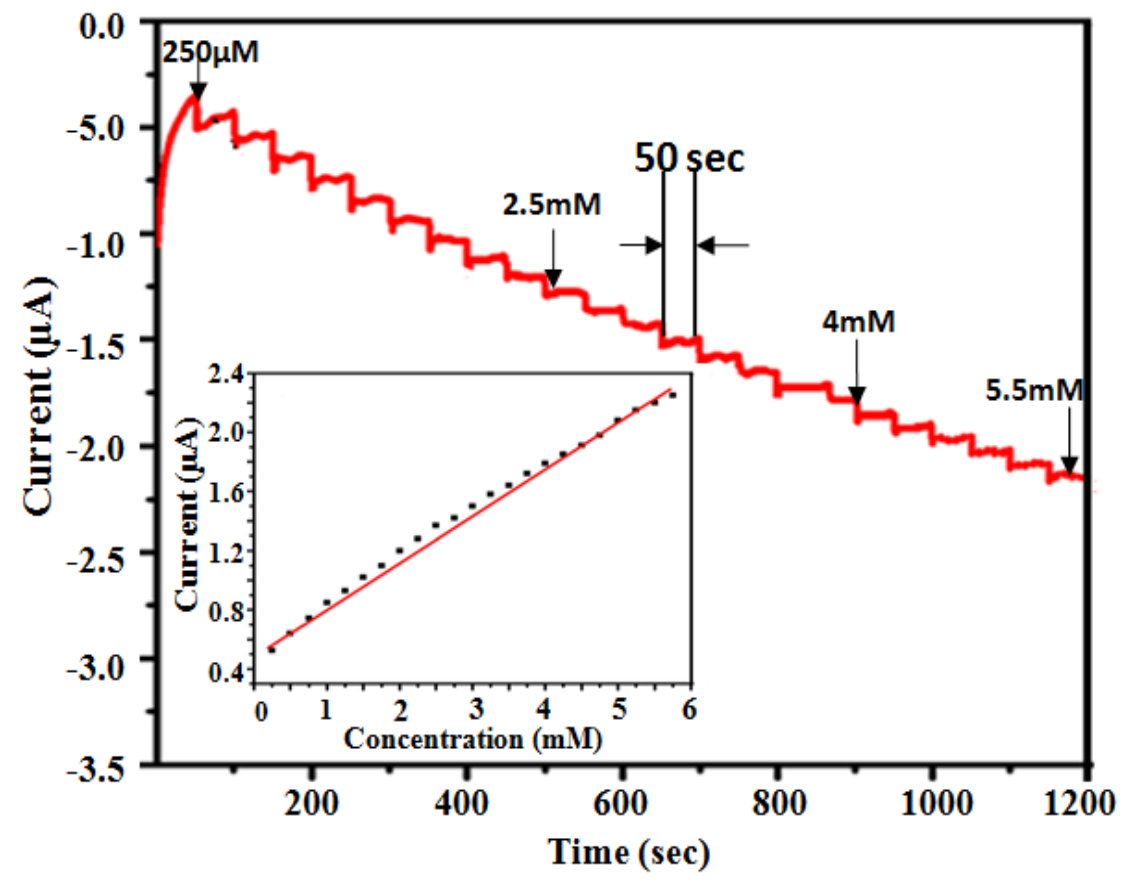

Figure 4.8. Amperometric response of graphene/carbon micropillar electrode array for successive additions of $250 \mu \mathrm{M}$ hydrogen peroxide into ( $\mathrm{pH}$ 7.2) phosphate buffer; Inset: Calibration curve of graphene/ carbon micropillar electrode array with different concentrations of $\mathrm{H}_{2} \mathrm{O}_{2}$. Operating potential: $+0.4 \mathrm{~V}$. 


\subsection{Conclusions}

In summary, a facile methodology to prepare graphene coated 3D substrates using ESD technique was demonstrated. The effect of ESD processing conditions on the morphology of the deposited graphene film was studied. The $\mathrm{C} / \mathrm{O}$ ratio of the deposited ratio was higher compared to raw graphene nanosheets due to thermal reduction at high deposition temperature. The graphene/ carbon micropillar electrode arrays showed faster charge transfer and higher electrochemical activity towards $\mathrm{H}_{2} \mathrm{O}_{2}$ compared to bare carbon micropillar electrode array. Our methodology promises a simple approach to coat nanomaterials onto functional microelectrodes with controllable morphology and employ them for electrochemical sensing.

\subsection{References}

[1] K.S. Novoselov, A. K. Geim, S. V. Morozov, D. Jiang, Y. Zhang, S. V. Dubonos, I. V. Grigorieva, A. A. Firsov, Electric Field Effect in Atomically Thin Carbon Films. 2004, Science, 306,666-669.

[2] K. S. Novoselov, A. K. Geim, S. V. Morozov, D. Jiang, M. I. Katsnelson, I. V. Grigorieva, S. V. Dubonos, A. A. Firsov, Two-dimensional gas of massless Dirac fermions in graphene. 2005, Nat. Lett., 2005, 438, 197-200.

[3] S. R. C. Vivekchand, C. S. Rout, K. S. Subrahmanyam, A. Govindraj, C. N. R. Rao, Graphene-based electrochemical supercapacitors. 2008, J. Chem. Sci, 120, 9-13.

[4] E.J. Yoo, J. Kim, E. Hosono, H.S. Zhou, T. Kudo, I. Honma, Large reversible Li storage of graphene nanosheet families for use in rechargeable lithium ion batteries. 2008, Nano Lett., 8, 2277-2282.

[5] H. Lee, J. Ihm, M.L. Cohen, S.G.Louie, Calcium-decorated graphene-based nanostructures for high-capacity hydrogen storage. 2012, Nano lett., 10, 793-798.

[6] Y. Xuan, Y.Q.Wu, T. Shen, M. Qi, M.A. Capano, J.A. Cooper, P.D. Ye, Atomiclayer-deposited nanostructures for graphene-based nanoelectronics. 2008, Appl. Phys. Lett., 92,013101 (3pp). 
[7] S. Park, J. An, J.W. Suk, R.S. Ruoff, Graphene-based actuators. 2010, Small, 6, 210-212.

[8] Y. Wang, Y. Shao, D.W. Matson, J. Li, Y. Lin, Nitrogen-doped graphene and its application in electrochemical biosensing. 2010, Nano Lett., 4, 1790-1798.

[9] T. Ramanathan, A.A. Abdala, S. Stankovich, D.A. Dikin, M.H. Alonso, R.D. Piner, D.H. Adamson, H.C. Schniepp, X. Chen, R.S. Ruoff, S.T. Nguyen, I.A. Aksay, R.K. Prud'Homme, L.C. Brinson, Functionalized graphene sheets for polymer nanocomposites.2008, Nat.Nano, 3, 327.

[10] J. Zhao, S. Pei, W. Ren, L.Gao, H. Cheng, Efficient Preparation of Large-Area Graphene Oxide Sheets for Transparent Conductive Films. 2010, ACS Nano, 4, 5245-5252.

[11] Y.Zhu, S. Murali, M.d. Stoller, K.J. ganesh, W. Cai, P.J. Ferreira, A. Pirkle, R.M. Wallace, K.A. Cychosz, M. Thommes, D. Su, E.A. Stach, R.S. Ruoff, Supercapacitors with a novel carbon produced by activation of graphene. 2011, Science, 332, 1537-1541.

[12] Q. He, S. Wu, S. Gao, X. Cao, Z. Yin, H. Li, P. Chen, H. Zhang, Transparent, flexible, all-reduced graphene oxide thin film transistors. 2011, ACS Nano, 5, 5038-5044.

[13] X. Wang, L. Zhi, K. Mullen, Transparent, Conductive Graphene Electrodes for Dye-Sensitized Solar Cells. 2008, Nano Lett., 8, 323.

[14] Q. Zhang, S. Yang, J. Zhang, L. Zhang, P. Kang, J. Li, J. Xu, H. Zhou, X. Song, Fabrication of an electrochemical platform based on the self-assembly of graphene oxide- multiwall carbon nanotubenanocomposite and horseradishperoxidase: direct electrochemistry and electrocatalysis. $\mathbf{2 0 1 1}$ Nanotechnology, 22, 494010323 (7pp).

[15] Q. Zeng, J. Cheng, L. Tang, X.Liu, Y. Liu, J. Li, J. Jiang. 2010, Adv. Mater., 20, 3362.

[16] L. Tang, Y. Wang, Y. Li, H. Feng, J. Lu, J. Li, Preparation, Structure, and Electrochemical Properties of Reduced Graphene Sheet Films. 2009, Adv. Mater., 19, 2782-2789.

[17] L.J. Cote, F. Kim, J. Huang, Langmuir-Blodgett assembly of graphite oxide single layers. 2009, J.Am.Chem.Soc., 131, 1043-1049. 
[18] G. Eda, G. Fanchini, M. Chhowalla, Large-area ultrathin films of reduced graphene oxide as a transparent and flexible electronic material. 2008, Nat. Nano, 3, 270-274.

[19] S. Gilke, S. Han, M. Wang, K.L. Wang, R.B. A. Kaner,4 A chemical route to graphene for device applications. 2007, Nano Lett., 7, 3394-3398.

[20] S. J. An, Y. Zhu, S. H. lee, M. D. Stoller, T. Emilsson, S. Park, A. Velamakanni, J. An, R.S. Ruoff, Thin Film Fabrication and Simultaneous Anodic Reduction of Deposited Graphene Oxide Platelets by Electrophoretic Deposition. 2010, J. Phys. Chem. Lett., 1, 1259.

[21] V.C. Tung, L.M. Chen, M. J. Allen, J.K. Wassei, K. Nelson, R.B. Kaner, Y. Yang, Low-temperature solution processing of graphene-carbon nanotube hybrid materials for high-performance transparent conductors. 2009, Nano Lett., 9, 1949-1955.

[22] R. W. Hart, H. S. White, B. Dunn, D. R. Rolison, 3-D. Microbatteries. 2003, Elec. Comm., 5, 120-123.

[23] C. Sharma, S. Patil, S. Saurabh, A. Sharma, R. Venkatragavan, Resorcinolformaldehyde based carbon nanospheres by electrospraying. 2009, Bull. Mater. Sci., 32, 239-246.

[24] W. Chen, M. Beidaghi, V. Penmatsa, L. Bechtold, L. Kumari, W.Z. Li, C. Wang, Integration of carbon nanotubes to C-MEMS for On-chip supercapacitors. 2010, IEEE trans on Nano., 9, 734-740.

[25] Y. P. Hsieh, M. Hofmann, H. Son, X. Jia, Y.F. Chen, C.T Liang, M.S. Dresselhaus, J. Kong. 2009, Nanotechnology, 20, 065601(6pp).

[26] Y.Yu, Y. Shi, C. Chen, C. Wang, Direct deposition of single-walled carbon nanotube thin films via electrostatic spray assisted chemical vapor deposition. 2008, J. Phys. Chem C, 112, 4176.

[27] A. Dhanabalan, Y. Yu, X. Li, K. Bechtold, C. Wang, Porous SnO2/CNT composite anodes: Influence of composition anddeposition temperature on the electrochemical performance. 2010, J. Mat. Res., 25, 1554-1560.

[28] M. Knez, K. Nielsch, L. Niinisto, Synthesis and surface engineering of complex nanostructures by atomic layer deposition. 2007, Adv. Mat., 19, 3425-3438.

[29] D. A. LaVan, P.M. George, R. Langer, Simple, Three-dimensional microfabrication of electrodeposited structures. 2003, Angew. Chem. Int. Ed., 42, 1262-1265. 
[30] Y. Xu, X. Zhu, Y. Dan, J.H. Moon, V. W. Chen, a. T. Johnson, J. W. Perry, S. Yang, Electrodeposition of $3 D$ titania photonic crystals from holographically patterned microporous polymer templates. 2008, Chem. Mater., 20, 1816-1823.

[31] M. Parodi, T. Batchelder, P. Haaland, J. McKibben, Spin coating and alternative techniques for flat panel displays. 1996, Semiconductor Int., 19, 101.

[32] A. Jaworek, Micro- and nanoparticle production by electrospraying. 2007, Powder Tech., 176, 18-35.

[33] C. Chen, E. M. Kelder, P. J. J. M. van der Put, J. Schoonman, Morphology control of thin $\mathrm{LiCoO}_{2}$ films fabricated using the electrostatic spray deposition (ESD) technique. 1996, J. Mater. Chem., 6, 765-771.

[34] T. Maitra, S. Sharmao, A. Srivastava, Y. Cho, M. Madou, A. Sharma, Improved graphitization and electrical conductivity of suspended carbon nanofibers derived from carbon nanotube/polyacrylonitrile composites by directed electrospinning. 2012, Carbon, 50, 1753-1761.

[35] C. Sharma, H. Katepalli, A. Sharma, M.Madou, Fabrication and electrical conductivity of suspended carbon nanofiber arrays. 2011, Carbon, 49, 1727-1732.

[36] S. Sharma, A. Sharma, Y. Cho, M.Madou, Increased graphitization in electrospun single suspended carbon nanowires integrated with carbon-MEMS and carbonNEMS platforms. 2011, Appl.Mater.Interfaces, 4, 34.

[37] V. Penmatsa, J. H. Yang, Y. Yu, C. Wang, Fabrication of porous carbon micropillars using a block copolymer as porogen. 2010, Carbon, 48, 4109-4115.

[38] B.Y. Park, L. Taherabadi, C. Wang, Electrical Properties and Shrinkage of Carbonized Photoresist Films and the Implications for Carbon Microelectromechanical Systems Devices in Conductive Media. 2005, J. Electrochem. Soc., 152, J136-J143.

[39] H.S. Min, B. Y. Park, L. Taherabadi, C. Wang, Y. Yeh, R. Zaouk, M.J. Madou, B. Dunn, Fabrication and properties of a carbon/polypyrrole three-dimensional microbattery, 2008, J. Power Sources, 178, 795-800.

[40] H. Xu, K. Malladi, C. Wang, L. Kulinsky, M. Song, M. Madou, Carbon postmicroarrays for glucose sensors. 2003, Biosens and Bioelec, 23, 1637-1644.

[41] J.H. Yang, V. Penmatsa, S. Tajima, H. Kawarada, C. Wang, Direct amination on 3-dimensional pyrolyzed carbon micropattern surface for DNA detection. 2003, Materials lett., 63, 2680-2683. 
[42] S. Ranganathan, R. McCreery, S.M. Majji, M. Madou, Photoresist-derived carbon for microelectromechanical systems and electrochemical applications. 2000, J. Elect. Soc., 147, 277-282.

[43] A.C. Ferrari, J. C. Meyer, V. Scardaci, C. Casiraghi, M. Lazzeri, F. Mauri, S. Piscanec, D. Jiang, K. S. Novoselov, S. Roth, A. K. Geim Raman Spectrum of Graphene and Graphene Layers. 2006, Phys. Rev. Lett., 97, 187401(4pp).

[44] F. Tuinstra, J.L. Koenig. Raman spectrum of graphite. 1970, J. Chem. Phys., 53, 112631.

[45] C. D. Zangmeister. Preparation and Evaluation of Graphite Oxide Reduced at $220{ }^{\circ} \mathrm{C} .2010$, Chem. Mater., 22, 5625-5629.

[46] D. Yang, A. Velamakanni, G. Bozoklu, S. Park, Meryl. Stoller, R. D. Piner, S. Stankovich, I. Jung, D. A. Field, C. A. Ventrice, R. S. Ruoff, Chemical analysis of graphene oxide films after heat and chemical treatments by X-ray photoelectron and Micro-Raman spectroscopy. 2009, Carbon, 47, 145-152.

[47] S. Yumitori, Correlation of Cls chemical state intensities with the Ols intensity in the XPS analysis of anodically oxidized glass-like carbon samples. 2000, J. Mater. Sci., 35, 139-146.

[48] D. Lee, V. R. Erigala, M. Dasari, J. Yu, R. M. Dickson, N. Murthy, Detection of hydrogen peroxide with chemiluminescent micelles. 2008, Int. J. Nanomedicine, 3, 471-476.

[49] M. S. Goh, M. Pumera,4 The electrochemical response of graphene sheets is independent of the number of layers from a single graphene sheet to multilayer stacked graphene platelets. 2010, J. Chem. Asian., 5, 2355-2357. 


\section{CHAPTER 5}

\section{WAFER-LEVEL FABRICATION OF FUNCTIONALIZED CARBON NANOSTRUCTURES WITH CONTROLLABLE SIZE, SHAPE AND POSITION}

\subsection{Introduction}

In the previous two chapters, surface engineered 3D carbon microstructure architectures were developed to increase the surface area of the electrodes in a given substrate footprint. Alternatively, one dimensional carbon nanostructures offer high surface to volume ratio, decreased charging currents and faster electrochemical reactions [1-3]. Till now, carbon nanotubes (CNTs) have attracted majority of the research efforts due to their unique physiochemical and electrical properties, but the success rate for CNT based- functional devices is only approximately 5\%. So in order to incorporate nanostructures in practical functional devices, two major issues still remain, i.e., (i) how to synthesize substantial quantities of nanostructures with controllable sizes and shapes at preferred locations; (ii) how to integrate nanostructures in devices that are feasible for mass production.

The ability to fabricate well-defined carbon microstructures with atomically smooth surfaces is made possible by pyrolyzing organic polymer precursors [4-8]. In this approach, also known as C-MEMS technique, polymers patterned by conventional photolithography or soft lithography are carbonized at high temperatures under inert ambience, causing the chain scission of organic compounds to form solid carbon. Since lithography techniques are used for patterning purpose, the electrodes obtained by this manner have better resolution and reproducibility when compared to the traditional casted carbon ink electrodes [9]. Such carbon structures have been used in applications 
such as on-chip supercapacitors [10], microbatteries [11], DNA detection [12], glucose sensors [13], and di-electrophoretic electrode arrays for the micromanipulation of microand nanoparticles [14]. In addition, different research groups have adapted this method to produce carbon architectures such as suspended nanowires, nanobridges and three dimensional (3D) carbon micropillars [15]. Recently, controlled carbon nanostructures have been fabricated by employing tools such as scanning electron beam lithography (EBL) [16] and ion-beam lithography [17] combined with pyrolysis. Although these procedures are capable of patterning sub-100nm features, they are not economically feasible for mass production due to their low inherent throughput, need for ultra-high vacuum systems, and high operating costs.

The emergence of photo nanoimprint lithography (P-NIL) has garnered attention as a potential low cost, high throughput technique for the fabrication of nano-scale features beyond the resolution of the traditional photolithography. In a typical P-NIL process, the patterned UV transparent mold is stamped onto a photo sensitive polymer and cured by ultra violet light to create a thickness contrast [18]. Anisotropic reactive ion etching (RIE) process is performed to remove the residual resist in the compressed areas and transfer the thickness contrast pattern onto the underlying resist and substrate. The versatility of the NIL process allows direct patterning of functional materials with desired physical properties at the wafer level [19]. In comparison, other nanopatterning techniques such as holographic lithography, extreme ultraviolet interference lithography (EUV-IL) and EBL comes with critical drawbacks. The holographic lithography and EUV-IL allow only for the fabrication of periodic features without alignment and the long writing times make EBL only suited for small-area patterning. Furthermore, in 
P-NIL, a transparent mold enables optical alignment with high accuracy. Also typically, carbon nanostructures must be coupled with microfabricated contact pads for electrical and electrochemical measurements which are made through a second lithographic step to deposit metal pads. In contrast, by combining P-NIL and pyrolysis process, structures with dimensions from nanometers to millimeters can be seamlessly integrated. Due to these characteristic advantages, nanoimprint lithography has been extensively used in photonics [20-24], organic electronics [25], magnetic devices [26-30], and biological applications [31-34]. However, to the best of our knowledge no research effort has been published to utilize nanoimprinting technique for fabricating carbon nanostructures.

In this chapter, the fabrication of carbon nanostructure arrays based on P-NIL and pyrolysis is reported. In this strategy, P-NIL patterning of photoresist over a nanoimprint resist, followed by an etch transfer step, provides patterned nanostructures that are then carbonized under inert atmosphere. The resulting carbon nanostructures have composition and electrical properties comparable to carbon derived from pyrolysis of SU-8 precursor widely used in carbon-based MEMS fabrication. Finally, the carbon nanostructures were functionalized using direct amination technique for potential use in bioanalytical devices. This versatile approach can address the need for high-throughput fabrication of functionalized carbon nanostructures with controllable properties at desired locations.

\subsection{Materials and Methods}

Reduced graphene nanosheets used in this work were obtained from Cheaptubes Inc, USA. 1,2 propanediol was purchased from Sigma Aldrich, USA. NANO ${ }^{\text {TM }}$ SU-8 and NANO $^{\mathrm{TM}}$ developer were purchased from Microchem, USA. 


\subsubsection{Fabrication Process}

4inch size silicon oxide wafers were spin-cleaned by acetone and methanol followed by a dehydration bake at $150{ }^{\circ} \mathrm{C}$ for $5 \mathrm{~min}$. At the beginning, a $200 \mathrm{~nm}$ thin coating of AR-UL-01 (nanoimprint resist) was carried out using Headway research ${ }^{\mathrm{TM}}$ (Garland, TX) photoresist spinner at $4000 \mathrm{rpm}$ for $45 \mathrm{sec}$ and baked at $180^{\circ} \mathrm{C}$ for $90 \mathrm{sec}$. For patterning the nanoimprint resist, a thin layer of AR-UV-01 (photoresist) is spincoated on the top of AR-UL-01 layer at $5000 \mathrm{rpm}$ for $7 \mathrm{sec}$. The P-NIL process was conducted using an OAI Model 800 (San Jose, CA) mask aligner equipped with OAI Nano Imprint Module. The compressed photoresist was exposed by a $365 \mathrm{~nm}$ mercury lamp for $60 \mathrm{sec}$ with light intensity at $900 \mathrm{~mJ} / \mathrm{cm}^{2}$ to crosslink the polymer chains. In our work, the nanoimprinting was done using a 5 in $\mathrm{x} 5$ in mask with the nanoimprinted area being 2 inch diameter circle. The actual pattern area containing arrays of assorted nanostructures in the nanoimprinted area was 1 in $\mathrm{x} 1$ in square. Following the patterning process, initially a 10:1 $\mathrm{CF}_{4}$ and $\mathrm{O}_{2}$ mixture at $150 \mathrm{mTorr}$ and $75 \mathrm{~W}$ etch was perfomed for $30 \mathrm{sec}$ to remove the residual photoresist followed by an oxygen plasma treatment at $50 \mathrm{~W}$ and $150 \mathrm{mTorr}$ for $100 \mathrm{sec}$ to etch the residual nanoimprint resist. The remaining photoresist layer on the top was then removed by a $10: 1 \mathrm{CF}_{4}$ and $\mathrm{O}_{2} \mathrm{RIE}$ for $60 \mathrm{sec}$ at 150 mTorr and $75 \mathrm{~W}$. The samples were carbonized in an alumina-tube furnace (Lindberg) by a two-step pyrolysis process. The samples were initially heated at $5{ }^{\circ} \mathrm{C} / \mathrm{min}$ rate from room temperature to $350{ }^{\circ} \mathrm{C}$, and held at that temperature for $40 \mathrm{~min}$ followed by ramping to $1000{ }^{\circ} \mathrm{C}$ and 60 min holding time. The samples were eventually cooled down slowly to the room temperature under constant gas flow. During the pyrolysis process, forming gas $\left(95 \% \mathrm{~N}_{2}+5 \% \mathrm{H}_{2}\right)$ was continuously flowed at $500 \mathrm{sccm}$. 


\subsubsection{Surface Functionalization}

Before the direct amination process, the samples were first thoroughly rinsed with DI water and then blow dried. The amination process was performed at room temperature in an ammonia gas $(99.9 \%)$ environment and using UV lamp (wavelength=253.7nm). Prior to UV irradiation, the reaction chamber was purged with nitrogen gas for $5 \mathrm{~min}$ to remove oxygen and other gases. The reaction chamber was then irradiated with UV light for $4 \mathrm{hr}$ under a continuous flow of ammonia gas at $100 \mathrm{sccm}$. Finally, nitrogen gas is purged for 5 min to remove any ammonia in the reaction chamber before removing the sample. The experimental setup is shown schematically in Figure 5.1.

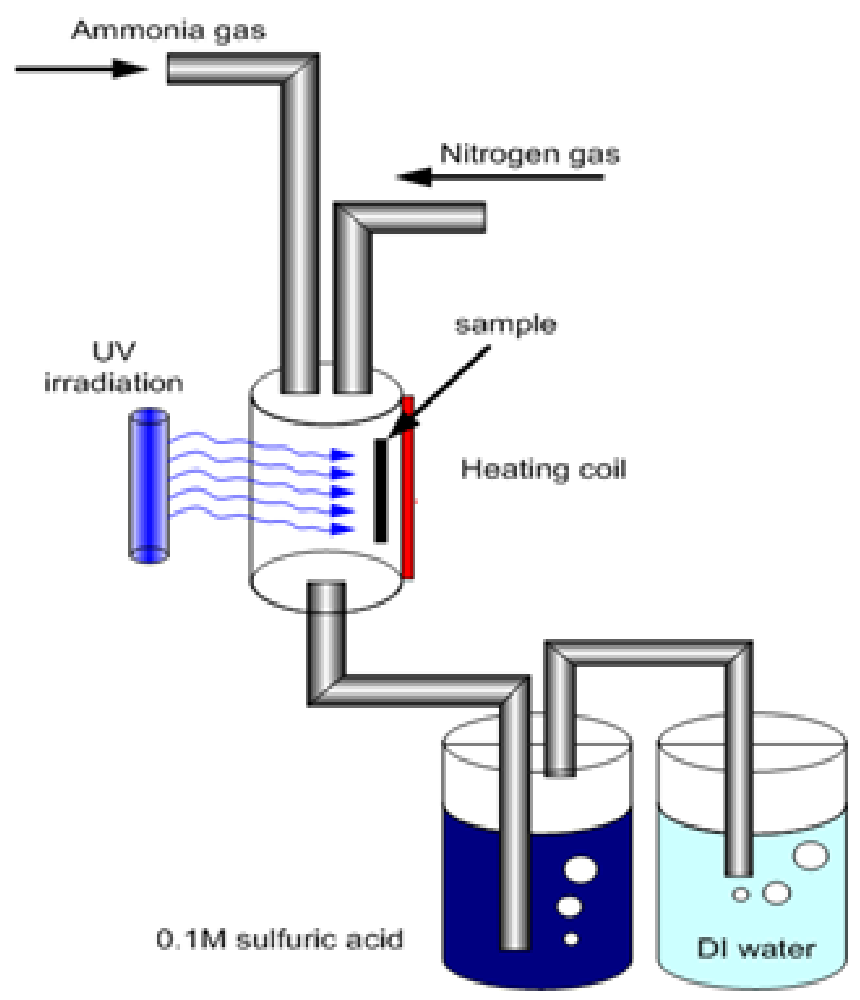

Figure 5.1. Schematic representation of the direct-amination functionalization process 


\subsubsection{Characterization}

Thermal behavior of AR-UL-01 was investigated by DSC-2910 and TGA-2950 (both from thermal Analysis Instruments, New Castle, DE) under an argon atmosphere. The heating cycle used for both DSC and TGA test is same as the pyrolysis cycle. The morphology of the carbon nanostructures was characterized by JEOL JSM-5335 scanning electron microscopy (SEM). In the case of non-conducting nanostructures, a thin gold film was evaporated on the top to avoid charging. The Raman spectra were measured at room temperature using a Dilor Raman Spectrometer equipped with a CCD camera and an optical microscope that provided a laser beam focus diameter of about $1 \mu \mathrm{m}$. Argonion laser $(514.5 \mathrm{~nm})$ was used as the source during measurement. The chemical composition of AR-UL-01 surface before and after carbonization was investigated by an Ulvac $\Phi 3300$ XPS (Ulvac-Phi) with an anode source providing Al Ka radiation. The electron takeoff angle was $45 \pm 3^{\circ}$ relative to the substrate surface and the $\mathrm{C} 1 \mathrm{~s}$ peak was chosen as the reference binding energy $(285 \mathrm{eV})$. The sheet resistance measurements were measured using an Agilent 4156C precision semiconductor parameter analyzer (Santa Clara, CA) connected with a 4-point probe station. It should be pointed out that unpatterned pyrolyzed resist films (PPFs) from AR-UL-01 and SU-8 using same pyrolysis conditions were used for characterization measurements. 


\subsection{Results and Discussion}

\subsubsection{Morphology}

A schematic illustration of the experimental process is shown in Figure 5.2. A hard mold with surface-relief features is stamped onto a bi-layer resist film of silicon containing UV -curable photoresist and poly (methyl methacrylate) (PMMA) - based nanoimprint resist. The primary reason to use bi-layer resist technique is, if both the pattern definition layer (photoresist) and the pattern transfer layer (nanoimprint resist) are the same, removing residual resist by dry etch may cause damage to the resist profile. To overcome this problem, in our work a bilayer resist film was used. In addition, the PMMA based nanoimprint layer in the bi-layer resist film provides good adhesion between the UV-curable photoresist and the underlying substrate. The critical difference between both the resists used in this work is the high contrast in etch selectivities and it is also important to note that the underlying nanoimprint resist is not sensitive to UV light and therefore cannot be patterned without the presence of photoresist. After the mold is stamped, the bi-layer resist film is irradiated with UV light in order to pattern the photoresist top layer. Subsequently, the pattern is transferred to nanoimprint resist via a two-step reactive ion etching. Initially a $\mathrm{CF}_{4}+\mathrm{O}_{2} \mathrm{RIE}$ etch is performed to remove the residual photoresist followed by $\mathrm{O}_{2}$ RIE to etch the residual imprint resist present between the photoresist pattern. Since the silicon containing UV-curable photoresist top layer is resistant to $\mathrm{O}_{2}$ RIE, there is negligible etching of the photoresist. Finally to completely remove the photoresist top layer before pyrolysis of imprint resist, a $\mathrm{CF}_{4}+\mathrm{O}_{2}$ etch is performed. It is critical to completely remove the photoresist top layer because the silicon present in the photoresist could form silicon carbide during pyrolysis which 
significantly affects the electrical conductivity of the carbon nanostructures. In the final step, the nanoimprint resist pattern is carbonized by heating the sample at high temperature under inert atmosphere along with a pre-carbonization bake. The structures were subject to pre-carbonization bake to ensure extensive crosslinking and to improve the adhesion between polymer and the substrate. This helps the nanoimprint resist structures to maintain their shape during carbonization.

(a) Imprint the mold to define features

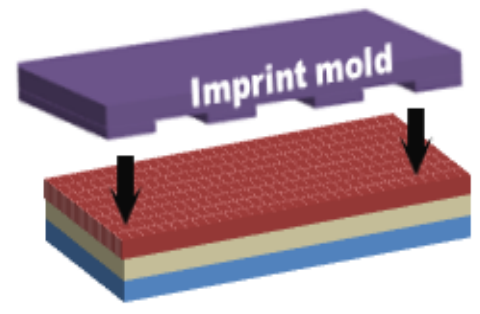

(d) $\mathrm{CF}_{4}+\mathrm{O}_{2} \mathrm{RIE}$ (Halogen etch)

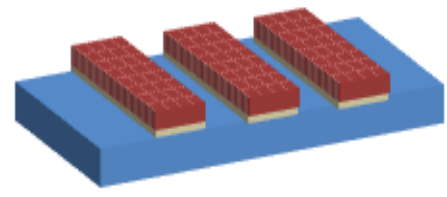

(b) Crosslinking the imprinted UV-sensitive polymer

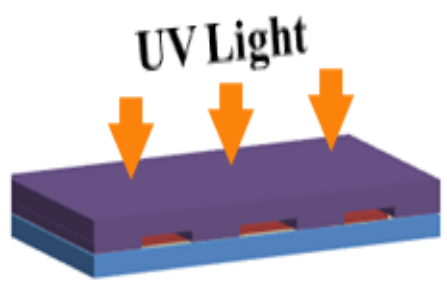

(e) $\mathrm{O}_{2}$ RIE followed by $\mathrm{CF}_{4}+\mathrm{O}_{2} \mathrm{RIE}$

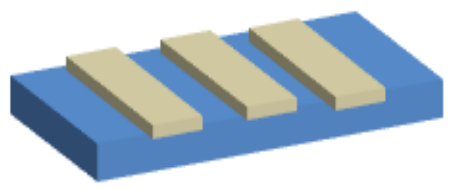

(c) Separating the mold to reveal thickness contrast

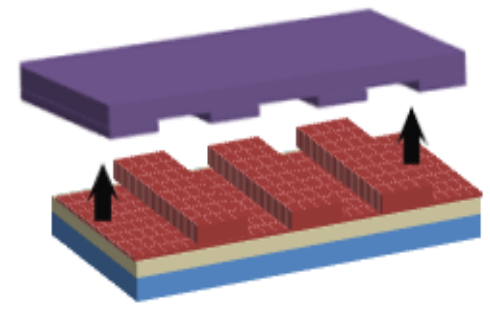

(f) Carbonization @ $1000^{\circ} \mathrm{C}$

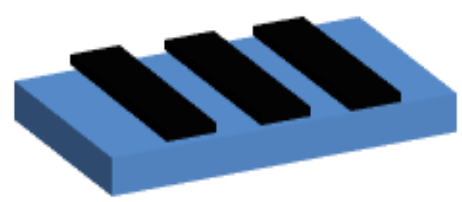

Figure 5.2. Schematic showing the fabrication process. a) Spin coat thin layers of nanoimprint resist and the photoresist on the substrate, b) Imprint a pre-designed glass mold to transfer the pattern onto the polymers and cure it with UV exposure, c) Retreat the glass mold to notice the pattern, d) Oxygen plasma treatment to etch residual nanoimprint resist, e) Etch the residual photoresist top layer by $\mathrm{CF}_{4}$ +oxygen reactive ion etch, and f) Carbonizing the polymer microstructures under forming gas atmosphere at high temperature.

Examples of carbon structures with different critical dimensions ranging from $50 \mathrm{~nm}$ to few microns fabricated using this methodology are shown in Figure 5.3a. 

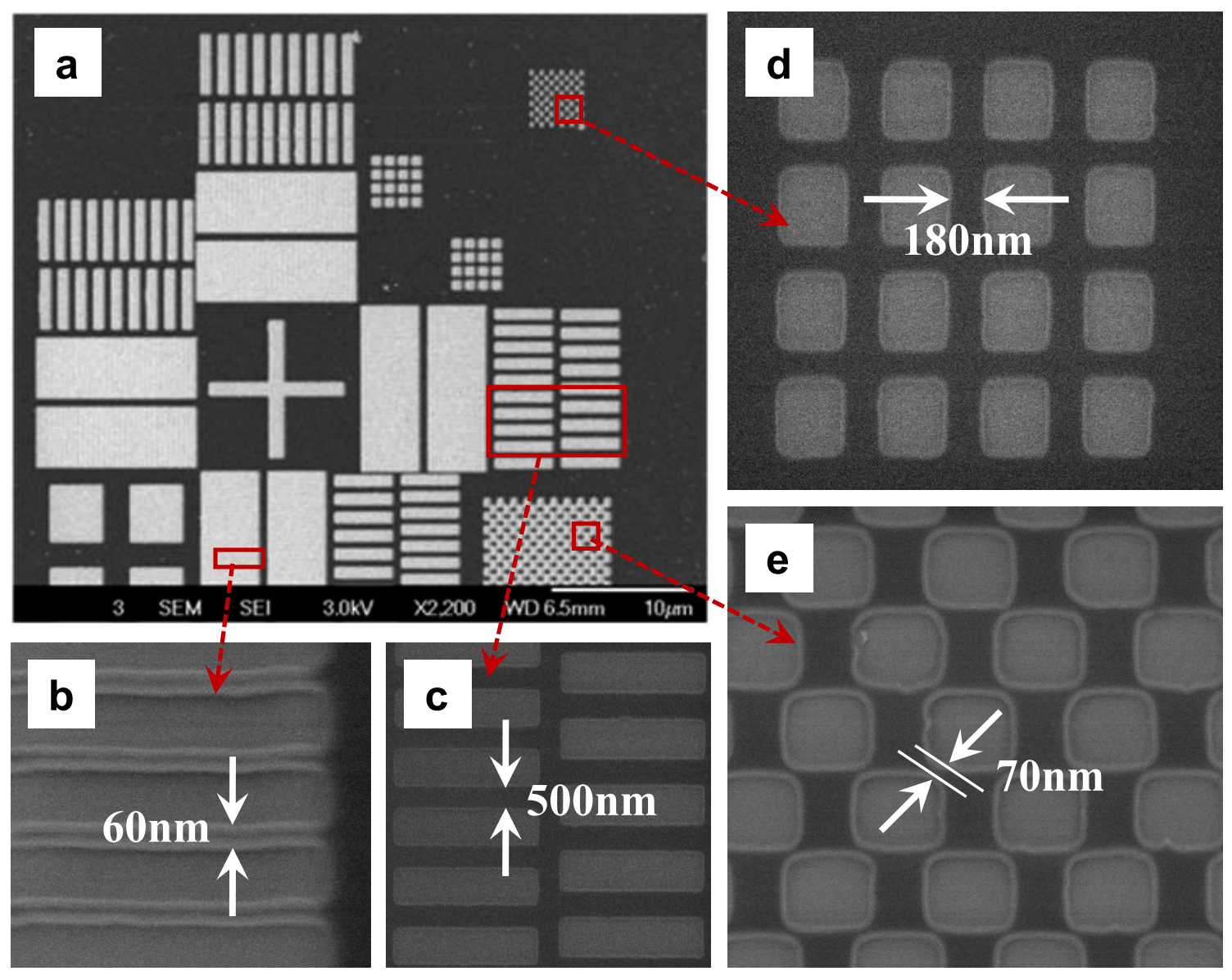

Figure 5.3. SEM images of carbon nanostructures. a) Carbon nanostructures with different designs patterns and feature sizes, b) 50-nm linear nanogratings, c) Comb design with 500nm pattern critical dimensions, d) Mesh pattern having $180 \mathrm{~nm}$ critical dimension features e) Chequered pattern with smallest feature size of $70 \mathrm{~nm}$ maintaining its lateral fidelity after pyrolysis.

The $50 \mathrm{~nm}$ nanogratings with spacing of $150 \mathrm{~nm}$ (Figure 5.3b) between them appear to be straight without visible defects. These linear nanogratings with nanometer dimensions can be fabricated with few millimeters length. Figure $5.3 \mathrm{c}$ illustrates the higher magnification SEM images of comb pattern with feature width of $500 \mathrm{~nm}$. The distance between each feature in this pattern is $660 \mathrm{~nm}$. The carbon pattern shown in Figure $5.3 \mathrm{~d}$ resembles a mesh structure with line widths of $200 \mathrm{~nm}$ horizontally and $300 \mathrm{~nm}$ 
vertically. The minute taperings at the edges could be attributed to the scattering of UV light during photocuring. High magnification SEM image of chequered pattern with the smallest feature size of $70 \mathrm{~nm}$ (Figure 5.3e) demonstrates that even structures with curved patterns maintain its lateral fidelity through the pyrolysis cycle. It is important to note that critical dimensions of the features shown here were limited by the mold available. As evident from these SEM images, the experimental approach greatly reduces the production time when compared to serial processing tools such as e-beam lithography EBL, ion-beam lithography due to the batch processing capability of P-NIL [35] and the nanostructures maintain good conformity through the pyrolysis cycle.

Carbonization of the nanostructures during the pyrolysis process usually involves a significant loss of material. Several reports have indicated the aromatization of polymer accompanied by the elimination of hydrogen and other heteroatoms during pyrolysis resulting in structure shrinkage. The removal of non-carbon species during carbonization induces densification of the polymer nanostructures and thus accounts for the volume shrinkage [36]. We investigated the vertical shrinkage of the nanoimprint resist by imaging linear structures of different widths before and after carbonization using atomic force microscopy (AFM). Figure 5.4 shows the AFM images that reveals a significant vertical shrinkage from $200 \mathrm{~nm}$ to $45 \mathrm{~nm}$. The $77.5 \%$ shrinkage that was observe here is similar to those recorded for carbon nanostructures derived from SU-8 2000.1 using EBL and pyrolysis [16]. Additionally, the lateral shrinkage calculated from SEM images (data not shown) exhibits a decrease of $5 \%$ or less. The reason for the minor shrinkage could be due to the strong adhesion between the nanoimprint resist and the Si substrate which forms a $\mathrm{SiC}$ interfacial layer during the pyrolysis process [16]. From the results, it can be 
pointed out that the lateral shrinkage of the carbon nanostructures is similar to that of the nanofeatures derived from SU-8 2000.1.

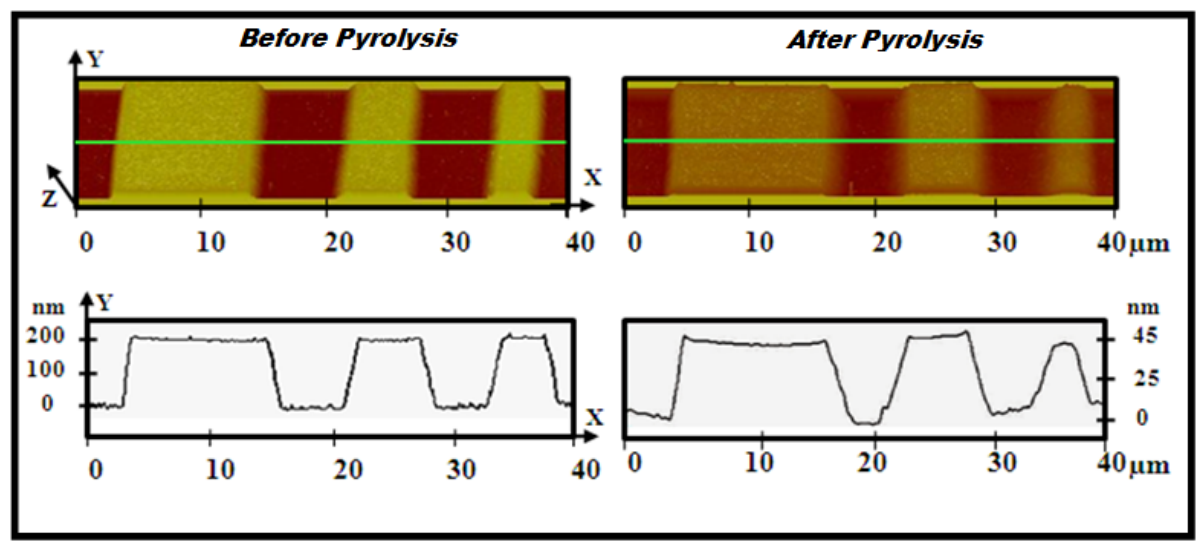

Figure 5.4. AFM images of the P-NIL patterned AR-UL-01 nanostructures a) Before and

b) After pyrolysis.

\subsubsection{Thermal Analysis}

The thermophysical analysis of the nanoimprint resist was investigated using differential scanning calorimetry (DSC) and thermogravimetric analysis (TGA) to understand the thermal behavior under pyrolysis cycle. The results were compared with negative SU-8 25 photoresist which is typically used as a precursor for pyrolyzed photoresist films in C-MEMS as shown in Figure 5.5. The nanoimprint resist used in this work is a PMMA (polymethyl methacrylate) based polymeric material whereas SU-8 25 photoresist is based on epoxy/phenolic resin. Region 1 in DSC curve shown in Figure 5.5a indicates a solvent loss cycle at temperatures below $250{ }^{\circ} \mathrm{C}$. During this process, the solvents in the resists evaporate along with the crosslinking reaction to harden the polymer film. The exothermal reaction peaks above $\sim 250^{\circ} \mathrm{C}$ (region 2 ) show the onset of different reactions which indicates the outgassing of byproducts. It is noteworthy that the exact reactions are difficult to confirm since the chemical composition of nanoimprint 
resist is not available in the datasheets. After $\sim 450{ }^{\circ} \mathrm{C}$, there are no obvious peaks indicating the completion of major outgassing and at this temperature solid carbon starts to form (region 3) [37]. In the case of SU-8, a sharp exothermic peak at $\sim 160^{\circ} \mathrm{C}$ (peak I) can be observed which is attributed to the beginning of the decomposition process of photoactive compound while the endothermic peak II at $\sim 190{ }^{\circ} \mathrm{C}$ indicates the thermal crosslinking of the resist [38]. The broad endothermic peak with its onset at $\sim 360{ }^{\circ} \mathrm{C}$ represents the evasion of $\mathrm{C}_{3}$ product gases. Additionally, it should be noted that during the pyrolysis process most of the degassing for both the resists occurs below $\sim 600{ }^{\circ} \mathrm{C}$. In Figure 5.5b, TGA curves show similar weight loss for both the resists. Analysis of the data shows a $20 \%$ weight loss starting at $\sim 50{ }^{\circ} \mathrm{C}$ which represents the evaporation of moisture and solvents in the resists. The weight change continued till the temperature reaches $\sim 300-350{ }^{\circ} \mathrm{C}$, where a sharp loss of $30-40 \%$ is observed. In this region most of the reactions occur concurrently outgassing byproducts. From $\sim 450{ }^{\circ} \mathrm{C}$ onwards the curves look fairly stable where the formation of solid carbon to expected to start. These results are in good agreement with the previously discussed DSC results.

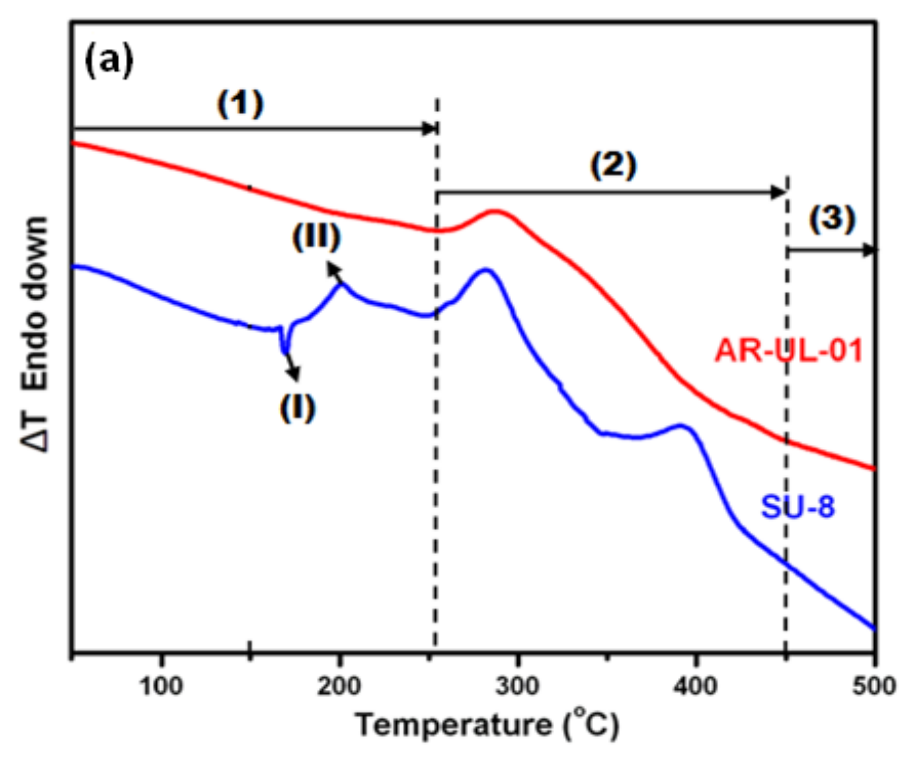




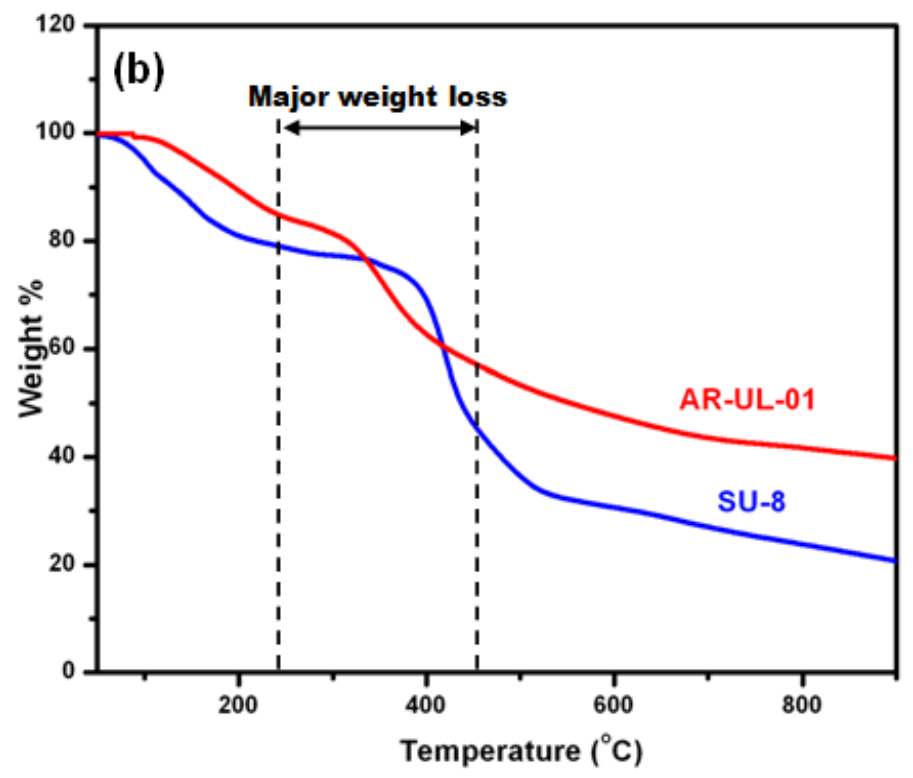

Figure 5.5. Thermal behavior of AR-UL-01 and SU-8 investigated from $50^{\circ} \mathrm{C}$ to $900^{\circ} \mathrm{C}$ by a) DSC and b) TGA graphs under inert atmosphere. In the curves, (1) Indicates the region where there is significant loss of solvents, (2) Decomposition of $\mathrm{C}_{3}$ and other byproducts occur (3) Temperature region where solid carbon forms; The peaks at (I) Indicate the decomposition of photoactive compound and (II) The exothermic peak due to the thermal crosslinking of the polymer.

\subsubsection{Raman Spectroscopy}

To elucidate the material microstructure and graphitization of the carbon nanostructures, the Raman spectra of carbonized nanoimprint resist and SU-8 films was investigated between $1000-1750 \mathrm{~cm}^{-1}$, shown in Fig 5.6. In this spectral range, both the graphitic band (G-band), and the disorder-induced band (D-band) can be detected [39]. The first peak at $1350 \mathrm{~cm}^{-1}$ is the disorder band of the microcrystallite graphite due to the enhanced double resonance Raman scattering. The second peak at $1590 \mathrm{~cm}^{-1}$ is close to the single Raman line found at $1575 \mathrm{~cm}^{-1}$ on single graphitic crystals that is ascribed to 
the bond stretching motion pairs of $\mathrm{sp}^{2} \mathrm{C}$ atoms present in the olefinic chains or the aromatic rings. The slight frequency shift $\left(\sim 15 \mathrm{~cm}^{-1}\right)$ observed in the spectrum could represent extremely small crystallite size [40]. Comparison of Raman spectra shows identical peak intensity ratios $\left(\mathrm{I}_{\mathrm{D}} / \mathrm{I}_{\mathrm{G}}\right)$ around 1.02 indicating similar microstructure for carbon samples produced from both nanoimprint resist and SU-8.

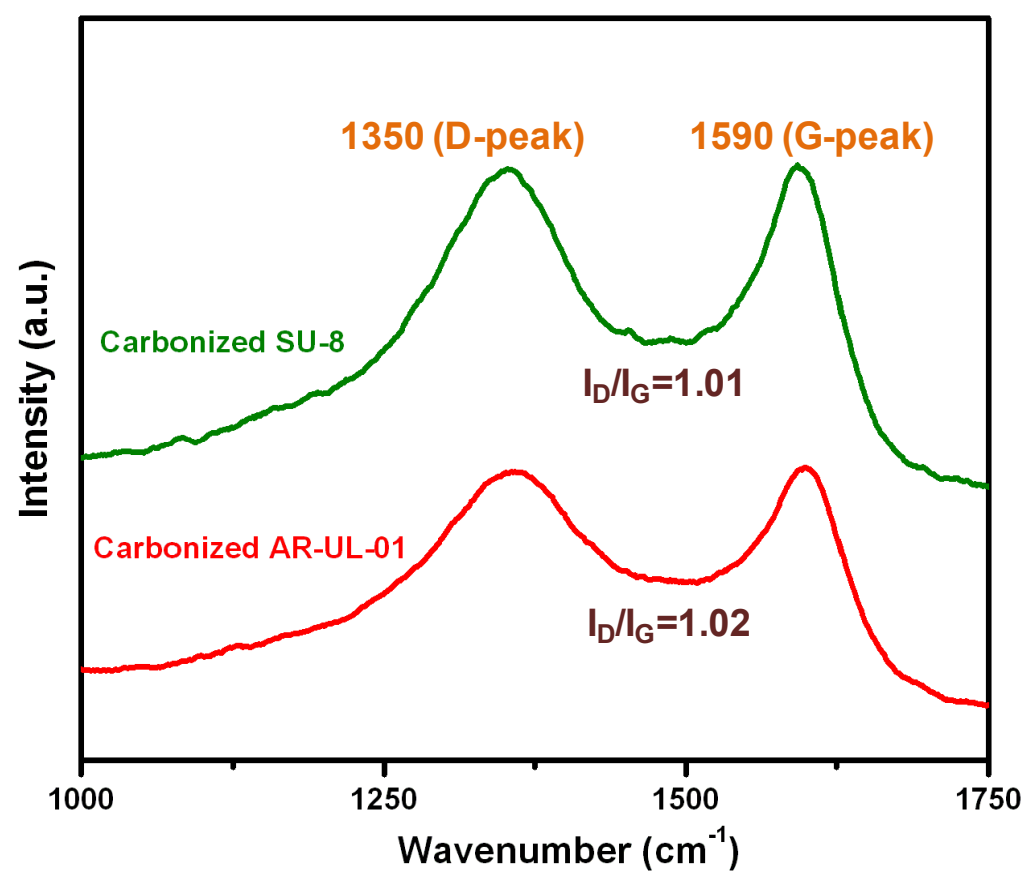

Figure 5.6. Raman spectra showing the comparison of thin carbon films prepared from AR-UL01 and SU-8.

\subsubsection{X-Ray Photoelectron Spectroscopy}

The elemental compositions in the XPS spectra are compared to evaluate the change in the chemical composition of AR-UL-01 with pyrolysis. For comparison purpose, both the as prepared and pyrolyzed nanoimprint resist samples were analyzed simultaneously. Three distinct peaks representing carbon $(284.6 \mathrm{eV})$, oxygen $(531.8 \mathrm{eV})$ and nitrogen $(398.4 \mathrm{eV})$ are evident in the spectra of AR-UL-01 before carbonization (Figure 5.7a). The shape and position of the high-resolution XPS spectra of carbon 
(Figure 5.7b), nitrogen (Figure 5.7c) and oxygen (Figure 5.7d) observed are consistent with previous publications [16]. However, the spectrum of the sample after carbonization exhibit only two peaks, i.e. carbon and oxygen peaks which can be explained by the fact that pyrolysis process accompanies extensive out-gassing of non-carboneous materials. For this reason, it can be observed that intensity of the carbon peak increased but at the same time the intensity of the oxygen peak has been significantly reduced. Also, no nitrogen peak was observed after pyrolysis. The main peak at $284.6 \mathrm{eV}$ indicates the carbon present in the precursor while the small shoulder at binding energy $\sim 286.1 \mathrm{eV}$ implies a contribution from the different bonding configurations of carbon and oxygen. After carbonization, it was observed that a significant decrease in the intensity of the oxygen peak and the peak at $\sim 286.1 \mathrm{eV}$ indicating that a significant out-gassing of oxygen supplements the pyrolysis process in inert atmosphere. The $\mathrm{O} / \mathrm{C}$ ratio calculated for uncarbonized AR-UL-01 showed $22.8 \pm 0.4$, but as expected the value reduced to $0.52 \pm 0.5$ after carbonization. However, the $\mathrm{O} / \mathrm{C}$ ratio of carbon is dependent on the precursor material used, synthesis technique and the post synthesis modification. For example, polished glassy carbon has a $7-20 \% \mathrm{O} / \mathrm{C}$ ratio [41], while vacuum heat-treated glassy carbon shows low $\mathrm{O} / \mathrm{C}$ ratios of $1-6 \%$ [42].
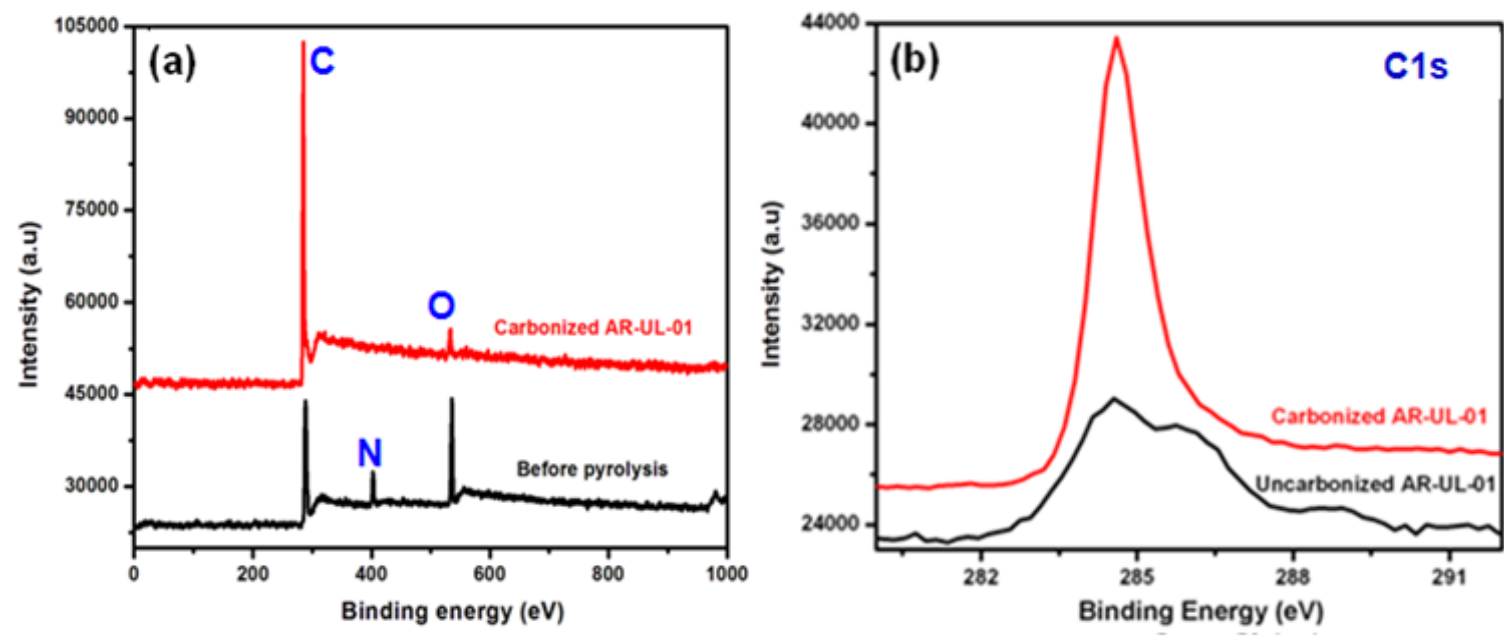

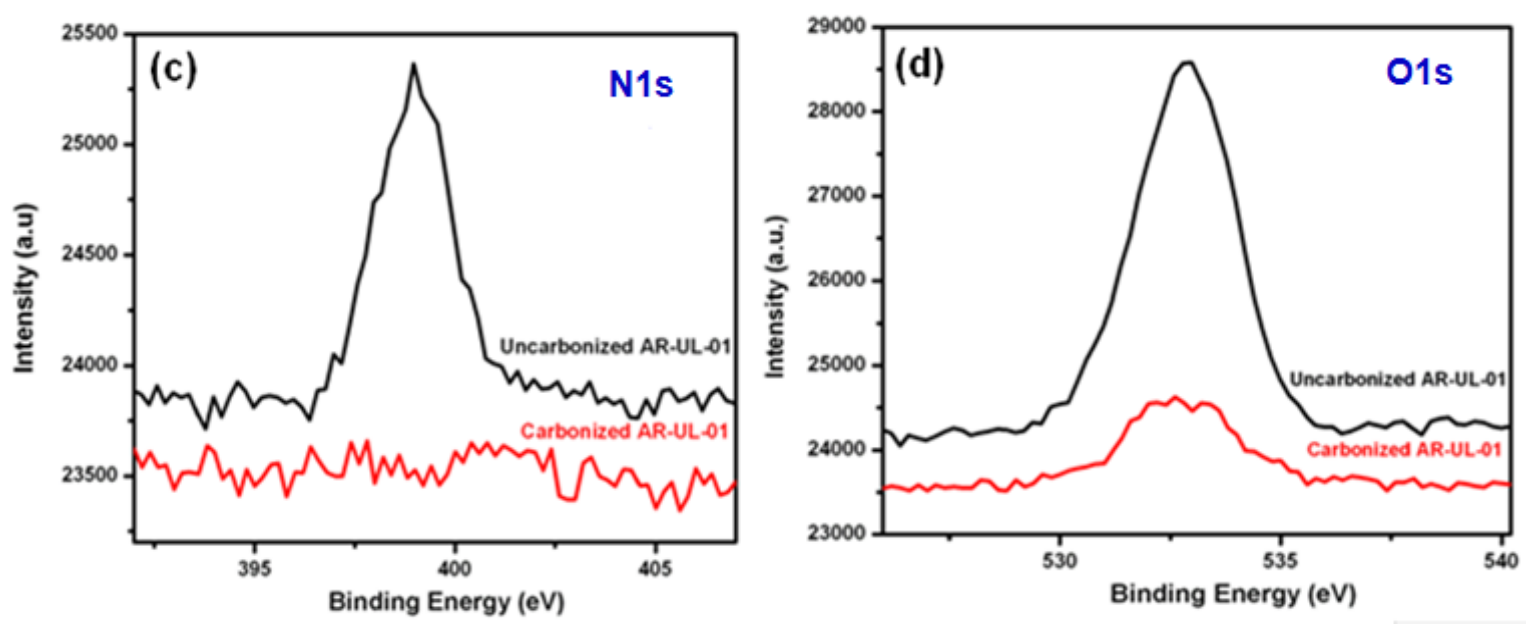

Figure 5.7. XPS spectra of AR-UL-01 before and after pyrolysis. a) Broad scan spectra, b) Carbon, c) Nitrogen d) Oxygen narrow peaks respectively.

\subsubsection{Resistivity}

The sheet resistivity was measured using a bridge structure by typical 4-point probe measurements [43]. The bar structure shown in Figure 5.8 has parameters of width $(\mathrm{W}=50$ microns) and length $(\mathrm{L}=2.5 \mathrm{~mm})$. Using this test structure, the sheet resistance was measured by flowing a current between pad $\mathrm{C}$ and $\mathrm{D}\left(\mathrm{I}_{\mathrm{CD}}\right)$ and measuring the voltage difference, $\left(\mathrm{V}_{\mathrm{AB}}\right)$ at pad $\mathrm{A}$ and $\mathrm{B}$. Note, in our experiment it is assumed that bar structure is homogeneous and any sidewall effects are negligible. The sheet resistance determined by using the equation (1) for carbonized AR-UL-01 yielded a value of $113.28 \Omega / \square$.

$$
R_{S b}=\frac{V_{B A}}{I_{C D}} \frac{W}{L}
$$

The resistivity $(\rho)$ calculated by sheet resistance times the film thickness for a 45 $\mathrm{nm}$ thick carbon film (which was measured by AFM) gives a value of $5.1 \times 10^{-4} \Omega . c m$, which is comparable to the resistivity $\sim 5 \times 10^{-5} \Omega . \mathrm{m}$ for glassy carbon prepared at $1000{ }^{\circ} \mathrm{C}[4,44]$. It is important to note that the resistivity of these nanostructures enables 
their use as nanoelectrodes in sensing, electrochemistry, and in other nanoscale applications.

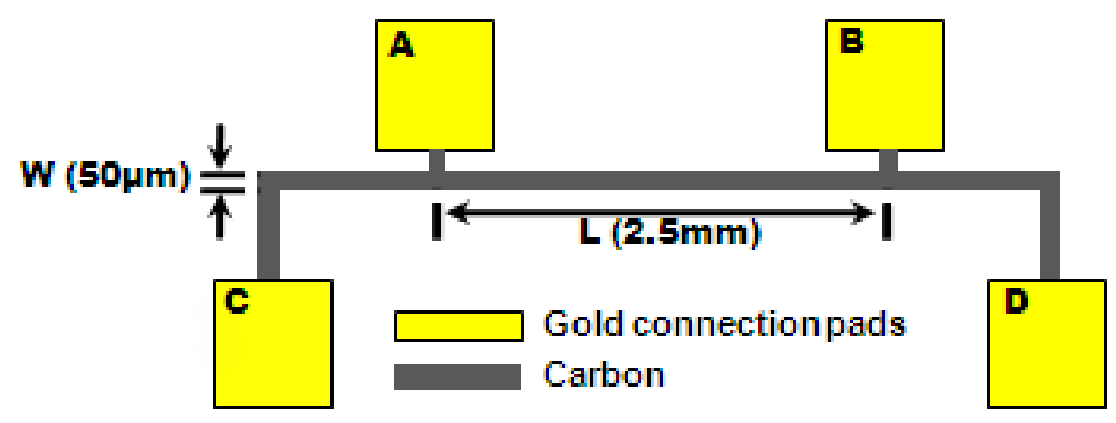

Figure 5.8. Schematic showing the test bridge structure used for measuring the sheet resistance.

\subsubsection{Surface Functionalization}

For potential use of carbon nanostructures in biosensors, termination on the carbon surface should be conducive to the interaction and immobilization of biomolecules [39]. Direct amination technique was used to graft amine-termination on the carbon which can covalently bind with carboxyl terminated biomolecules. In one of our previous publications, we examined this functionalization process on carbon derived from a negative-tone photoresist, SU-8 and used the platform for DNA detection [12]. In this work, the possibility of amine termination on the AR-UL-01 derived carbon by direct amination technique was evaluated. Figure 5.9 shows the broadscan XPS spectra of carbon surface treated at different amination times. Careful analysis of the XPS spectra for carbon film without amination shows only two major peaks at $284.6 \mathrm{eV}$ and $531.8 \mathrm{eV}$ which corresponds to carbon and oxygen respectively. But after amination, three distinct peaks representing carbon $(284.6 \mathrm{eV})$, oxygen $(531.8 \mathrm{Ev})$ and nitrogen $(398.4 \mathrm{eV})$ are evident. As the amination treatment time increased, the intensity of the nitrogen peak also increased correspondingly until it reaches saturation at 4 hrs. Previous studies have shown 
that the nitrogen peak visible after amination is a result of ammonia gas forming $\mathrm{C}-\mathrm{NH}_{2}$ on the carbon substrate [12].

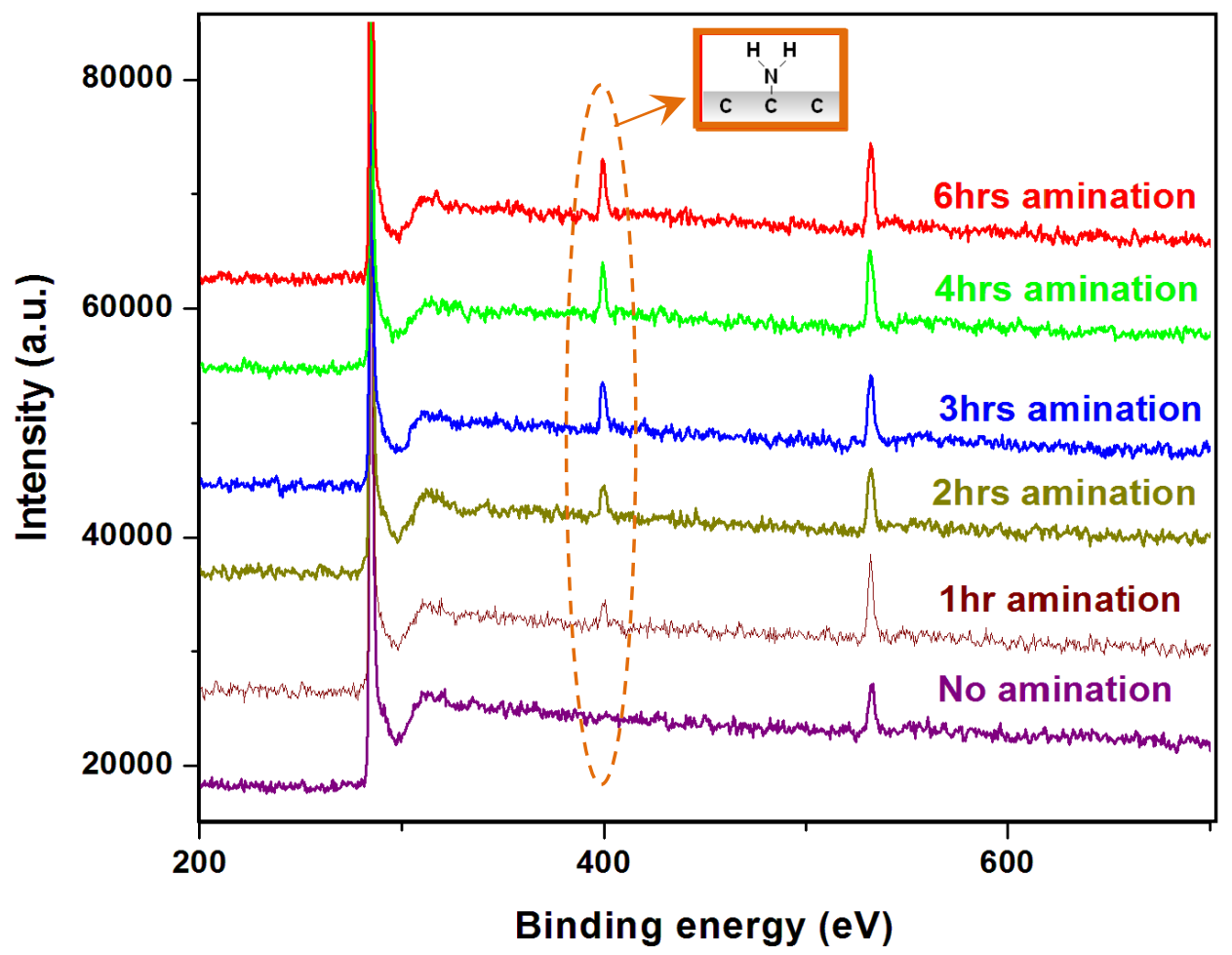

Figure 5.9. Summary of broadscan XPS spectra of carbon surface functionalized by direct amination technique at different treatment times.

High resolution XPS spectra of carbon surface before and after amination is shown in Figure 5.10. A significant nitrogen peak can be observed at $398.4 \mathrm{eV}$ after $6 \mathrm{hrs}$ amination. Alternatively, for untreated sample no peak was visible indicating the absence of any physically absorbed nitrogen. This confirms that nitrogen peak visible after amination is covalent binding of nitrogen with carbon which can used for subsequent immobilization of carboxyl-terminated biomolecules. 


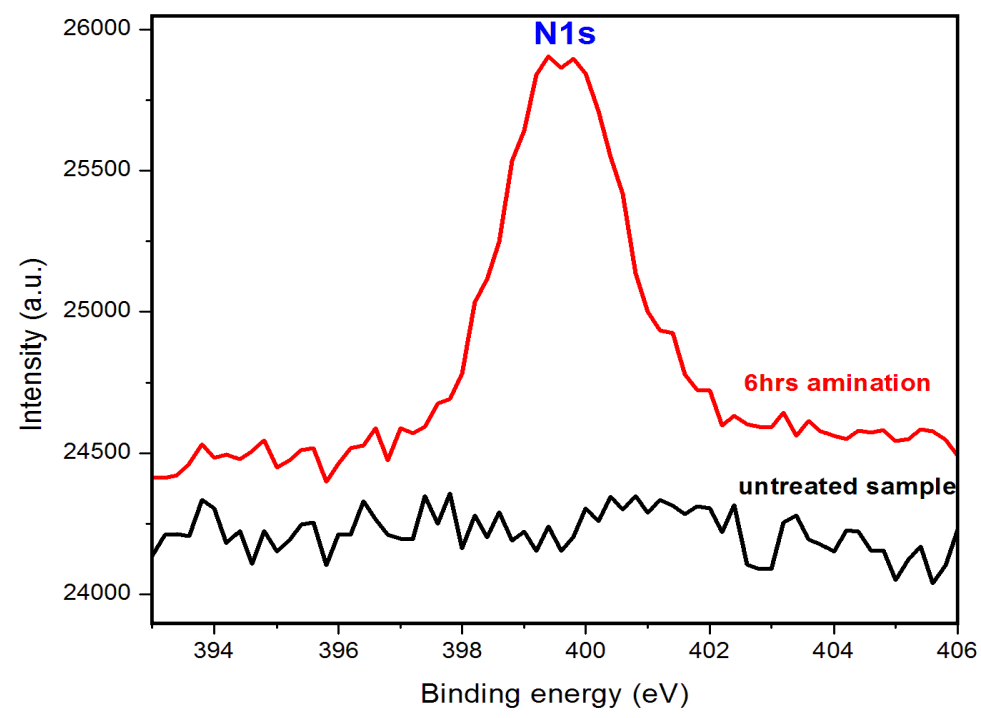

Figure 5.10. Comparison of high resolution XPS spectra of carbon surface before and after $6 \mathrm{hrs}$ direct amination treatment.

\subsection{Conclusions}

For the first time, a fabrication technique for patterning carbon nanostructures using P-NIL and pyrolysis was demonstrated. The carbonized nanostructures exhibit microstructure comparative to the glassy carbon materials produced by pyrolyzing organic materials. As expected the elemental composition in the nanoimprint resist changed with pyrolysis due to the ejection of non-carboneous species. Additionally, the electrical properties exhibited by carbon nanostructures are comparable to bulk glassy carbon. The carbon nanostructures were functionalized using direct amination technique. It was observed that the intensity of the nitrogen peak representing $\mathrm{C}-\mathrm{NH}_{2}$ bonds increased proportionally with time until it reaches saturation at $4 \mathrm{hrs}$. Controllable carbon features with nano/micro scale dimensions conceived by this cost-effective high throughput manufacturing technique will allow us to explore their use in functional nanodevices. 


\subsection{References}

[1] Y. Xia, P. Yang, Y. Sun, P. Wu, B. Mayers, B. Gates, Y. Yin, F. Kim, H. Yan, One-dimensional nanostructures: synthesis, characterization, and applications. 2003, Adv. Mater., 15 353-389.

[2] D. W. M. Arrigan, Nanoelectrodes, nanoelectrode arrays and their applications. 2004, Analyst, 129, 1157-1165.

[3] P. M. Ajayan, O. T. Zhou, Applications of carbon nanotubes. 2001, Top. Appl. Phys., 80 391-425.

[4] C. Wang, G. Jia, L. Taherabadi, M. Madou, A novel method for the fabrication of high-aspect ratio C-MEMS structures. 2005, IEEE J. Microelectromech. Syst., 14, 348-358.

[5] S. Ranganathan, R. McCreery, S. M. Majji, M. Madou, Photoresist-derived carbon for microelectrochemical applications. 2000, J. Electrochem. Soc., 147, 277-282.

[6] A. Singh, J. Jayaram, M. Madou, S. Akbar, Pyrolysis of negative photoresists to fabricate carbon structures for microelectromechanical systems and electrochemical applications. 2002, J. Electrochem. Soc. 149 E78-E83.

[7] O. J. A. Schueller, S. T. Brittain, C. Marzolin, G. M. Whitesides, Fabrication and characterization of glassy carbon MEMS. 1997, Chem. Mater., 9, 1399-1406.

[8] O. J. A. Schueller, S. T. Brittain, G.M. Whitesides, Fabrication of glassy carbon microstructures by pyrolysis of microfabricated polymeric precursors. 1997, Adv. Mater., 9, 477-480.

[9] J. A. Lee, S. Hwang, J. Kwak, S. I. Park, S. S. Lee, K. C. Lee, An electrochemical impedance biosensor with aptamer-modified pyrolyzed carbon electrode for labelfree protein detection. 2008, Sensors Actuators B, 129, 372-379.

[10] W. Chen, M. Beidaghi, V. Penmatsa, K. Bechtold, L. Kumari, W.Z. Li, C. Wang, Integration of carbon nanotubes to C-MEMS for on-chip supercapacitors. 2010, IEEE Trans. Nanotechnol., 9, 734-739.

[11] C. Wang, L. Taherabadi, G. Jia, M. Madou, Y. Yeh, B. Dunn, C-MEMS for the manufacture of $3 D$ microbatteries. 2004, Electrochem. Solid-State Lett., 7, A435A438. 
[12] J. H. Yang, V. Penmatsa, S. Tajima, H. Kawarada, C. Wang, Direct amination on 3-dimensional pyrolyzed carbon micropattern surface for DNA detection.2009, Mater. Lett., 63, 2680-2683.

[13] H. Xu, K. Malladi, C. Wang, L. Kulinsky, M. Song, M. Madou, Carbon postmicroarrays for glucose sensors. 2008, Biosens. Bioelectron., 23, 1637-1644.

[14] Z. R. Tang, M. R. Malik, T. L. Shi, W. Lai, S. Liu, Modelling and fabrication of 3$D$ carbon-MEMS for dielectrophoretic manipulation of micro/nanoparticles in fluids. 2009, Mater. Sci. Forum, 628, 435-440.

[15] V. Penmatsa, J. H. Yang, Y. Yu, C. Wang, Fabrication of porous carbon micropillars using a block copolymer as porogen. 2010, Carbon, 48, 4109-4115.

[16] R. Du, S. Ssenyange, M. Aktary, M. T. McDermott, Fabrication and characterization of graphitic carbon nanostructures with controllable size, shape, and position. 2009, Small, 5, 1162-1168.

[17] P. Lemoine, S.S. Roy, J.P. Quinn, P.D. Maguire, J.A.D Mclaughlin, Carbon nanostructures grown with electron and ion beam methods. 2007, Appl. Phys., A86, 451-456.

[18] S. Y.Chou, P.R. Krauss, P.J. Renstrom, Nanoimprint lithography. 1996, J. Vac. Sci. Technol. B, 14, 4129-4133.

[19] C. Subramani, Y. Ofir, D. Patra, B.J. Jordan, I.W. Moran, M.H. Park, K.R. Carter, V.M. Rotello, Nanoimprinted polyethyleneimine: a multimodal template for nanoparticle assembly and immobilization. 2009, Adv. Funct. Mater., 19, 29372942.

[20] D. Pisignano, L. Persano, E. Mele, P. Visconti, M. Anni, G. Gigli, R. Cingolani, L. Favaretto, G. Barbarella, First-order imprinted organic distributed feedback lasers. 2005, Synth. Met., 153, 237-240.

[21] D. Pisignano, L. Persano,M.F. Raganato, P. Visconti, R. Cingolani, G. Barbarella, L. Favaretto, G. Gigli, Room-temperature nanoimprint lithography of nonthermoplastic organic films. 2004, Adv. Mater, 16, 525-529.

[22] L. J. Guo, X. Cheng, C.Y. Chao, Fabrication of photonic nanostructures in nonlinear optical polymers. 2002, J. Mod. Opt., 49, 663-673.

[23] X. Cheng, Y. T. Hong, J. Kanicki, L.J. Guo, High-resolution organic polymer light-emitting pixels fabricated by imprinting technique. 2002, J. Vac. Sci. Technol. B, 20, 2877-2880. 
[24] P.C. Kao, S.Y. Chu, T. Y. Chen, C. Y. Zhan, F.C. Hong, C.Y. Chang, L.C. Hsu, W.C. Liao, M.H. Hon, Fabrication of large-scaled organic light emitting devices on the flexible substrates using low-pressure imprinting lithography. 2005, IEEE Trans. Electron Devices 52 1722-1726.

[25] C.C. Cedeno, Nanoimprint lithography for organic electronics. 2002, Microelectron. Eng., 61, 25-31.

[26] S. Y.Chou, Patterned magnetic nanostructures and quantized magnetic disks. 1997, Proc. IEEE, 85, 652-671.

[27] W. Wu, B. Cui, X. Y. Sun, W. Zhang, L. Zhuang, L.S. Kong, S.Y. Chou, Large area high density quantized magnetic disks fabricated using nanoimprint lithography. 1998, J. Vac. Sci. Technol. B, 16, 3825-3829.

[28] J. I. Martin, J. Nogues, K. Liu, J.L. Vicent, I.K. Schuller, Ordered magnetic nanostructures: Fabrication and properties. 2003, J. Magn. Mater., 256, 449-501

[29] T. Glinsner, P. Hangweier, H. Luesebrink, P. Dorsey, A. Homola, D. Wachenschwanz, Nanoimprint lithography enables patterned tracks for highcapacity hard disks. 2005, Solid State Technol., 15, 51-54.

[30] G.M. McClelland, M.W. Hart, C. T. Rettner, M.E. Best, K.R. Carter, B.D. Terris, Nanoscale patterning of magnetic islands by imprint lithography using a flexible mold. 2002, Appl. Phys. Lett., 81, 1483-1485.

[31] H. Cao, Z.N. Yu, J. Wang, J.O. Tegenfeldt, R.H. Austin, E. Chen, W. Wu, S.Y. Chou, Fabrication of $10 \mathrm{~nm}$ enclosed nanofluidic channels. 2002, Appl. Phys. Lett., 81, 174-176.

[32] L.J. Guo, X. Cheng, C.F. Chou, Fabrication of size-controllable nanofluidic channels by nanoimprinting and its application for DNA stretching. 2004, Nano Lett., 4, 69-73.

[33] J.D. Hoff, L.J. Cheng, E. Meyhofer, L.J. Guo, A.J. Hunt, Nanoscale protein patterning by imprint lithography. 2004, Nano Lett., 4, 853-857.

[34] D. Falconnet, D. Pasqui, S. Park, R. Eckert, H. Schift, J. Gobrecht, R. Barbucci, M. Textor, A novel approach to produce protein nanopatterns by combining nanoimprint lithography and molecular self-assembly. 2004, Nano Lett., 4, 19091914.

[35] S. Y. Chou, P.R. Krauss, P.J. Renstrom, Imprint of sub-25 $\mathrm{nm}$ vias and trenches in polymers. 1995, Appl. Phys. Lett., 67, 3114-17. 
[36] O.J.A. Schueller, S.T. Brittain, G.M. Whitesides, Fabrication of glassy carbon microstructures by soft lithography. 1999, Sensors Actuators A, 72, 125-39.

[37] D. Rodriguezponce, K. Lozano, T. Eubanks, H. Ahmad, D. Ferrer, Y. Lin, Thermophysical analysis of SU8-modified microstructures created by visible light lithography. 2010, J. Polym. Sci. Polym. Phys. B, 48, 47-54.

[38] J. Hammacher, A. Fuelle, J. Flaemig, J. Saupe, B. Loechel, J. Grimm, Stress engineering and mechanical properties of SU-8-layers for mechanical applications. 2008, Microsyst. Technol., 14, 1515-1523.

[39] H. Chun, M.G. Hahm, Homma, Y.R Meritz, K. Kuramochi, L. Menon, L. Ci, P.M. Ajayan, Y.J. Jung, Engineering low- aspect ratio carbon nanostructures: nanocups, nanorings, and nanocontainers. 2009, ACS Nano, 3, 1274-1279.

[40] F. Tuinstra, J.L. Koenig, Raman spectrum of graphite. 1970, J. Chem. Phys., 53, $1126-1130$.

[41] P. Chen, R.L. McCreery, Control of electron transfer kinetics at glassy carbon electrodes by specific surface modification. 1996, Anal. Chem., 68, 3958-3965.

[42] D.T. Fagan, I.F. Hu, T. Kuwana, Vacuum heat-treatment for activation of glassy carbon electrodes. 1985, Anal. Chem., 57, 2759-2763.

[43] S. Enderling, C.L. Brown, S. Smith, M.H. Dicks, J.T.M Stevenson, M. Mitkova, M. Kozicki, A.J. Walton, Sheet resistance measurement of non-standard cleanroom materials using suspended greek cross test structures. 2006, IEEE Trans. Semicond. Manuf., 19. 2-9.

[44] B.Y. Park, L. Yaherabadi, C. Wang, J. Zoval, M. Madou, Electrical properties and shrinkage of carbonized photoresist films and the implications for carbonmicroelectromechanical systems devices in conductive media. 2005, J. Electrochem. Soc., 152, J136-J143. 


\section{CHAPTER 6}

\section{COMPARISON OF DIFFERENT OXIDATION TREATMENTS ON C-MEMS DERIVED CARBON}

\subsection{Introduction}

One of the pivotal factors to improve the stability and detection performance of biosensors is to find a suitable substrate for conjugating target specific bioreceptors and their attachment chemistry [1]. An ideal substrate is chemically robust, easily functionalized, and compatible with a wide variety of analytical modalities (fluorescence, surface plasmon resonance, microscopy, electrochemistry, etc). So parallel with the development of micro/nano-electrode architectures using nanotechnology and MEMS techniques, considerable attention is also being concentrated on studying various nano/bio interfaces for improved bioassays.

Glass, silicon and gold substrates are commonly used due to their well-defined functionalization methods: the silanization of glass, silicon and the formation of selfassembled monolayers (SAMs) on gold. Alternatively, carbon-based materials are considered attractive, since they offer good electrical conductivity, better resistance towards biofouling and superior stability over conventional substrates when exposed to prolonged incubations in aqueous solutions at elevated temperatures and/or serial hybridizations. The more important aspect of using carbon-based materials is that their surfaces can be easily modified using physical, chemical or electrochemical techniques.

Recently, microfabrication of carbonaceous material has received a lot of attention due to many applications that can be envisioned such as microelectrodes in biosensing devices, electrochemical sensors and miniaturized energy storage/energy 
conversion devices. Microfabrication technology based on carbonaceous materials can greatly extend the practical application of MEMS in biosensing. However, to take advantage of the benefits of pyrolyzed carbon in biosensors, the surface of carbon needs to be properly functionalized with chemical groups to attach a biologically derived material such as recombinant antibodies, engineered proteins, aptamers etc. to detect a wide variety of physiological substances.

Many different oxidation techniques are known and have been used for functionalizing pyrolyzed carbon, such as wet chemical techniques [2], oxygen-plasma or atom-beam treatments [3,4], hot-filament techniques [5], thermal oxidation in oxygen atmosphere [6,7], photochemical procedures [8], ozone exposure [9] or electrochemical oxidation $[6,10,11]$. However, it is often quite difficult to compare the different oxidation methods due to the different analysis techniques, setups and evaluation procedures used in the various studies. An interesting question is whether different oxygen-containing groups are produced by different oxidation techniques or exposures [12]. Furthermore, the result of the oxidation process (with respect to both the amount of oxygen as well as type of carbon-oxygen groups) may also depend on the nature of the carbon surface.

\subsection{Materials and Methods}

\subsubsection{Vacuum Ultraviolet (VUV) Surface Treatment}

Figure 6.1 shows the schematic illustration of the VUV surface treatment system (UER 20-172 from Ushio, Inc.) and the actual instrument used in this work. The VUV system uses a xenon excimer (Xe ) lamp to generate a ultraviolet (VUV) light with a central wavelength of $172 \mathrm{~nm}$. The working principle of the VUV surface treatment is extensively discussed in Ref [13]. 


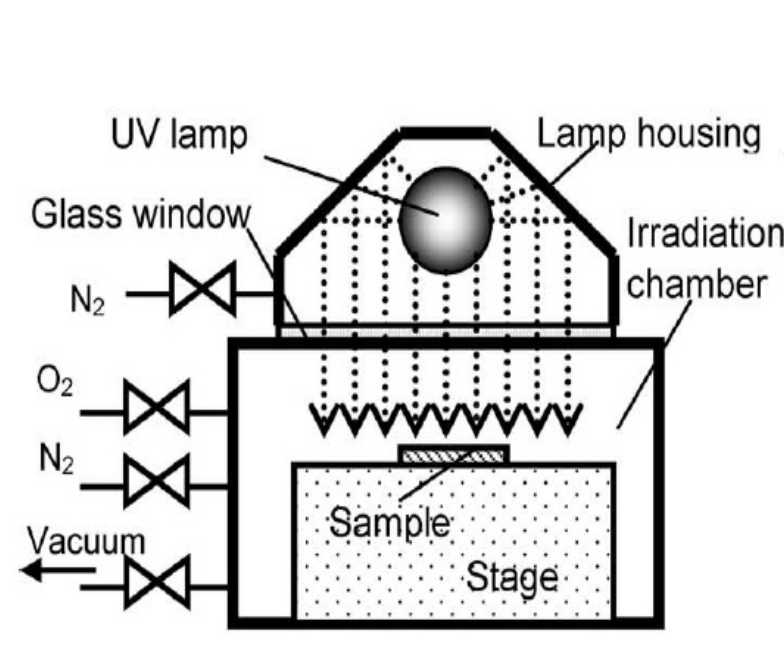

(a)

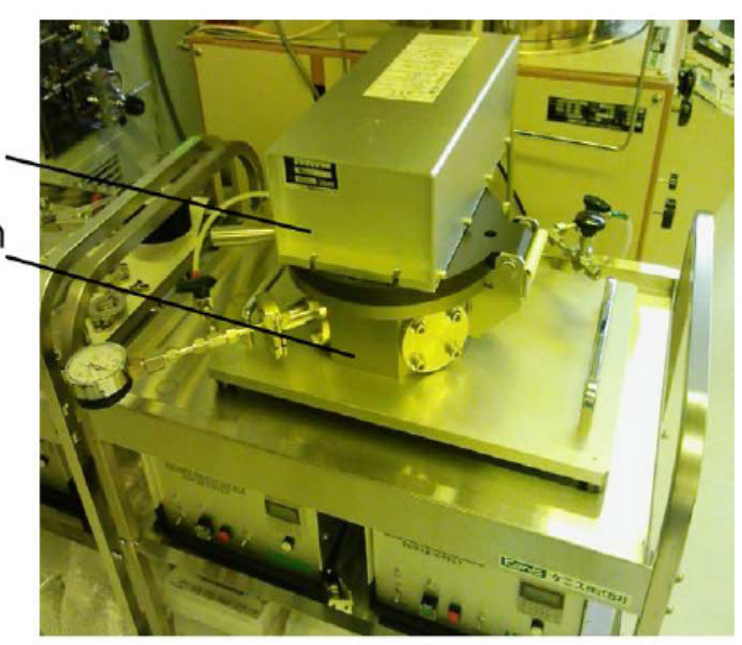

(b)

Figure 6.1. (a) Schematic showing the VUV surface treatment system, (b) Actual UER 20-172 VUV system. Figure adapted from Ref [13].

After the sample was inserted, the chamber was evacuated and oxygen gas $\left(\mathrm{O}_{2}\right)$ was introduced, waiting till it reaches the required pressure of $3.0 \times 10^{4} \mathrm{~Pa}$. In this work, VUV treatment was done at $20 \mathrm{~W}$ lamp power and light intensity $12 \mathrm{~mW} / \mathrm{cm}^{2}$ time for times varying from 15-120 $\mathrm{min}$. the whole treatment process was done at room temperature. The excimer light is transmitted through the glass window of the lamp housing and chemical reaction chains are triggered in the irradiation chamber.

The reactions at the atomic and molecular level responsible for excimer and excited oxygen generation are

$$
\mathrm{Xe}_{2}{ }_{2} \rightarrow \mathrm{Xe}+\mathrm{Xe}+\mathrm{h} v
$$

followed by

$$
\begin{gathered}
\mathrm{O}_{2}+\mathrm{h} v \longrightarrow 2 \mathrm{O} \\
\mathrm{O}_{2}+\mathrm{O} \longrightarrow \mathrm{O}_{3} \\
2 \mathrm{O}_{3}+\mathrm{h} v \longrightarrow 3 \mathrm{O}_{2}
\end{gathered}
$$




\subsubsection{Electrochemical Activation}

Electrochemical activation of carbon is considered an attractive pretreatment technique, since it produces reproducible surface and shows improved electron transfer. The electrode can be electrochemically activated in either basic or acidic (or neutral) solution. Previous reports have shown that anodic oxidation of graphitic materials in aqueous solutions creates surface oxides primarily consisting of carboxylic and phenolic groups [14]. The composition of these oxides can be controlled to some extent by proper choice of current and potential profiles and also by the electrolyte solution. However, larger background current and higher fraction of oxygen contents have been observed for the electrode pretreated in acidic (or neutral) solutions.

To perform electrochemical activation the C-MEMS electrodes was connected with a piece of copper wire. Then the contact pad and the silver wire were fully covered by epoxy resin to prevent their exposure to the electrolyte. Subsequently, the sample was configured as the working electrode in a three-electrode system. The reference and the counter electrodes used were $\mathrm{Ag} / \mathrm{AgCl}$ and a Pt wire, respectively. Figure 6.2 shows the photograph of actual setup used for electrochemical activation. The activation process was performed in $0.5 \mathrm{M} \mathrm{H}_{2} \mathrm{SO}_{4}$ solution deaerated by nitrogen bubbling for time intervals between $5-30$ mins. A voltage of $1.9 \mathrm{~V}$ was applied to the electrodes for the durations ranging from 10-30 min using a multichannel potentiostat/galvanostat (VMP3, Princeton Applied Research). The electrodes were then negatively polarized at $-0.3 \mathrm{~V}$ for $10 \mathrm{~min}$. After electrochemical pretreatment, the electrodes were washed with DI water. 


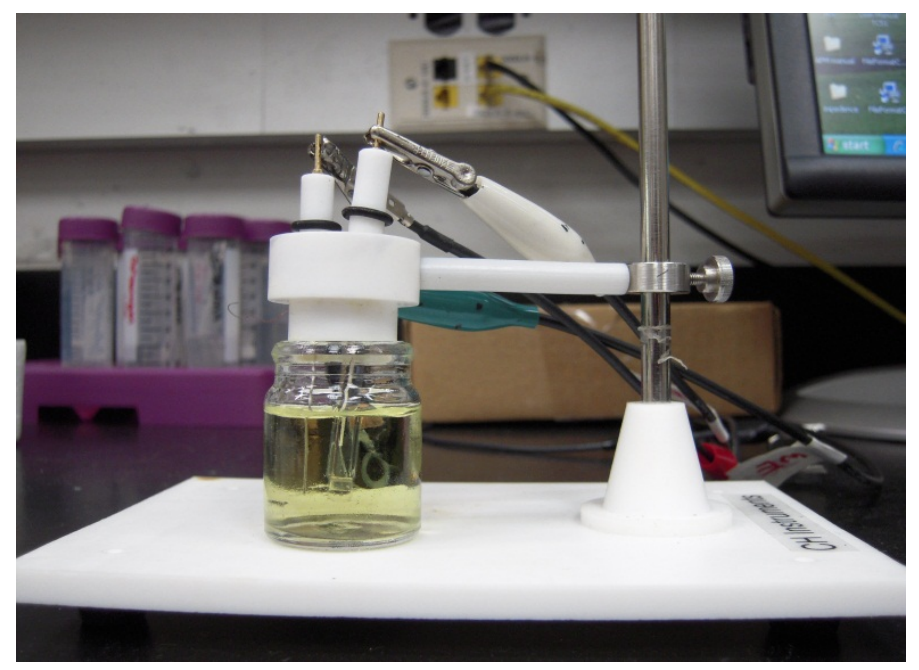

Figure 6.2. Photograph of the actual setup used for electrochemical activation

\subsubsection{UV/Ozone $\left(\mathrm{O}_{3}\right)$ treatment}

Ozone $\left(\mathrm{O}_{3}\right)$ is a powerful oxidant due to its ready dissociation into $\mathrm{O}_{2}$ and $\mathrm{O}$ radicals. In the photosensitized oxidation incorporating $\mathrm{UV} / \mathrm{O}_{3}$, the molecules are excited or dissociated by the absorption of short-wavelength UV radiation. The carbon atoms (preferably at the defect sites on carbon surface) react with the atomic oxygen from the continuous dissociations of oxygen molecules to generate ozone molecules for the carboxylation reaction to take place [15]. The major wavelengths of the ultraviolet rays radiated from a low-pressure mercury vapor lamp are $184.9 \mathrm{~nm}$ and $253.7 \mathrm{~nm}$. When atmospheric oxygen $\mathrm{O}_{2}$ is irradiated with ultraviolet rays with a wavelength of $184.9 \mathrm{~nm}$, the oxygen absorbs the ultraviolet rays to form $\mathrm{O}_{3}$ by the reactions (1) and (2)

$$
\begin{aligned}
& \mathrm{O}_{2} \rightarrow \mathrm{O}+\mathrm{O} \\
& \mathrm{O}+\mathrm{O}_{2} \rightarrow \mathrm{O}_{3}
\end{aligned}
$$

Ozone $\mathrm{O}_{3}$ irradiated with UV iradiation (wavelength of $253.7 \mathrm{~nm}$ ) absorbs the UV light to decompose $\mathrm{O}_{3}$. During the process of formation or decomposition of $\mathrm{O}_{3}$, atomic 
oxygen $\mathrm{O}$ having a strong oxidizing ability is generated which oxidizes the carbon surface.

The UV/ozone pretreatment was performed by using UV ozone cleaner UVy253 (Nippon Laser and Electronics Laboratory). At first, the reaction chamber was purged with nitrogen gas for $5 \mathrm{~min}$ to remove any active gases. Subsequently oxygen $\left(\mathrm{O}_{2}\right)$ gas is introduced for 5 mins. After turning of the oxygen gas supply, the UV light was turned on for times ranging from 10-180min. Finally, after turning of the UV source, nitrogen gas was introduced for $5 \mathrm{~min}$ to purge the ozone in the reaction chamber before opening the chamber door.

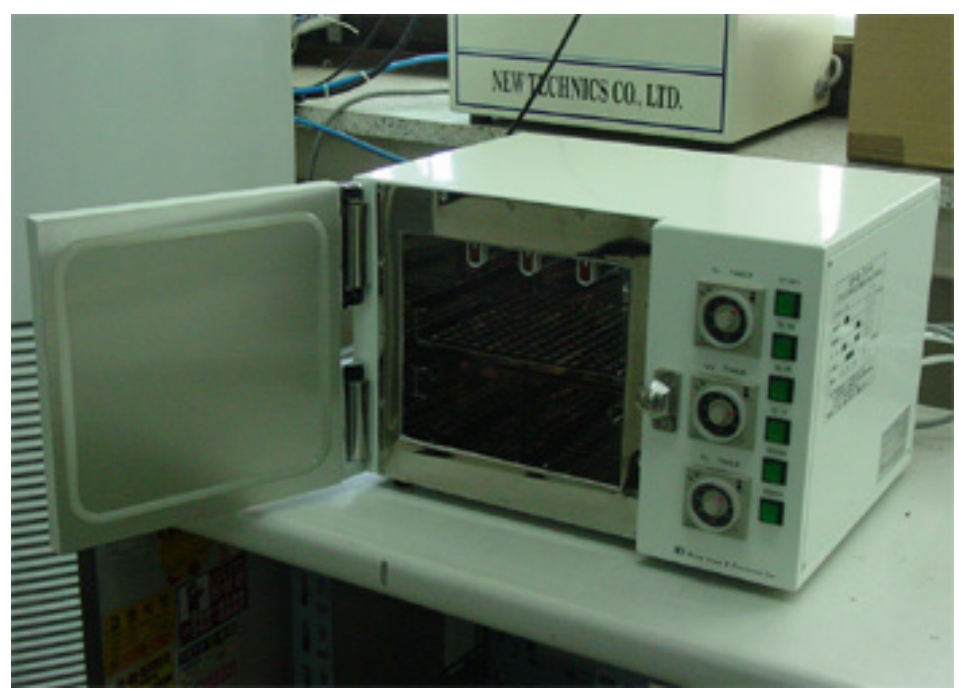

Figure 6.3. The actual $\mathrm{UVy} 253 \mathrm{UV} / \mathrm{O}_{3}$ surface pretreatment system

\subsubsection{Oxygen RIE pretreatment}

Previously the use of reactive, non-equilibrium oxygen plasmas as a means of introducing oxygen-containing functional groups onto the isotropic pyrolytic graphite surfaces have been investigated with promising results $[3,4]$. This pretreatment has been shown to incorporate carboxyl groups along with other surface oxides in a rapid, 
contaminant-free fashion. The other advantage of using this pretreatment on carbon surface is the ability to create surface porosity which potentially increases the effective surface area.

MARCH CS-1217 RIE system (shown in Figure 6.42) was used to treat the pyrolyzed carbon surface with oxygen plasma. This system has parallel plate reactor equipped with $13.65 \mathrm{MHz}$ RF source. The gas line for oxygen was been completely evacuated before the process to remove any moisture. The oxygen RIE time was varied from 1-10mins

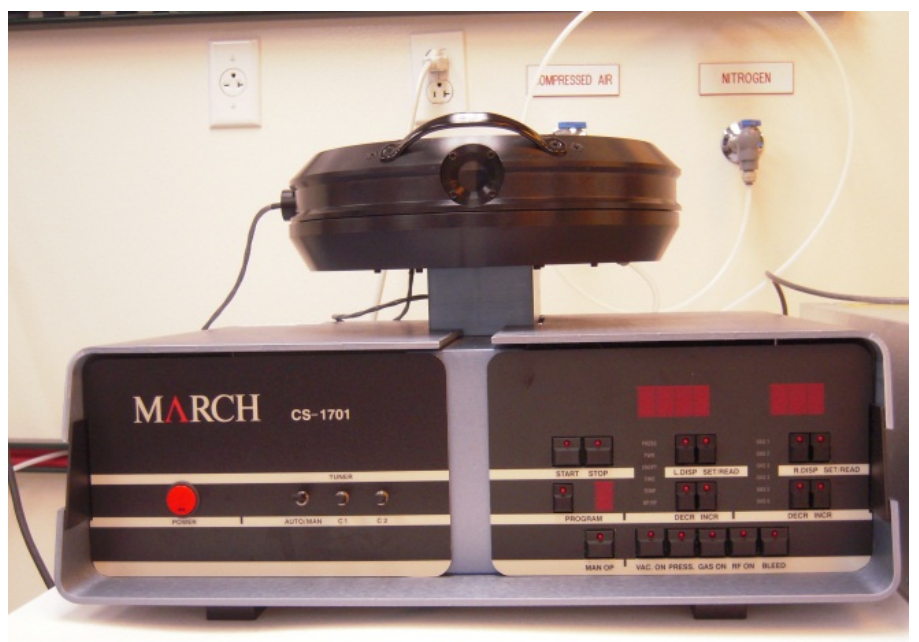

Figure 6.4. Photograph of the MARCH CS-1217 RIE system

\subsubsection{XPS Analysis}

The XPS analysis was investigated by an Ulvac $\Phi 3300$ XPS (Ulvac-Phi) with an anode source providing $\mathrm{Al} \mathrm{K} \alpha$ radiation. The electron takeoff angle was $45 \pm 3^{\circ}$ relative to the substrate surface and the C1s peak was chosen as the reference binding energy $(284.6 \mathrm{eV})$. Spectrum fitting routine was done with the following constraints: Shirley background was used. All peak contributions are mixed Gauss-Lorentz product functions with identical shapes and widths. 
In this work, we present a comparison of four different oxidation techniques applied to C-MEMS derived carbon films. Samples were investigated by X-ray photoelectron spectroscopy (XPS). XPS allows to quantify the oxygen concentration on the surface and to deduce information on the various carbon-oxygen groups present on the surface by analysis of the chemical shift of the C1s core level. Specifically we will focus on the following questions: (i) Do the various oxidation techniques yield different amounts of adsorbed oxygen?; (ii) Which types of carbon-oxygen groups are found on the surface and do they differ between the various oxidation techniques?

\subsection{Results and Discussion}

Figure 6.5 depicts the summary of measured oxygen content as a function of oxidation time in the case of all the four oxidation techniques. The oxidation levels saturate after treatment for longer duration in the case of VUV and ozone pretreatment compared to when oxidized by electrochemical activation (EA) and oxygen RIE pretreatments. These results are consistent with the fact that $\mathrm{VUV}$ and $\mathrm{UV} / \mathrm{O}_{3}$ are milder oxidation techniques which only show a minor increase in the surface roughness after treatment. Alternatively, in the case of both EA and oxygen RIE, as the pretreatment time was increased significant increase in the surface porosity and surface roughness was observed (results not shown). It is noteworthy that as the ECA and oxygen RIE pretreatment time increased over $30 \mathrm{mins}$ and $10 \mathrm{mins}$ respectively, the films started to peel/etch from the substrate and the results were not reproducible. Thus, the achieved level of oxidation cannot be increased further without completely destroying the carbon films. 


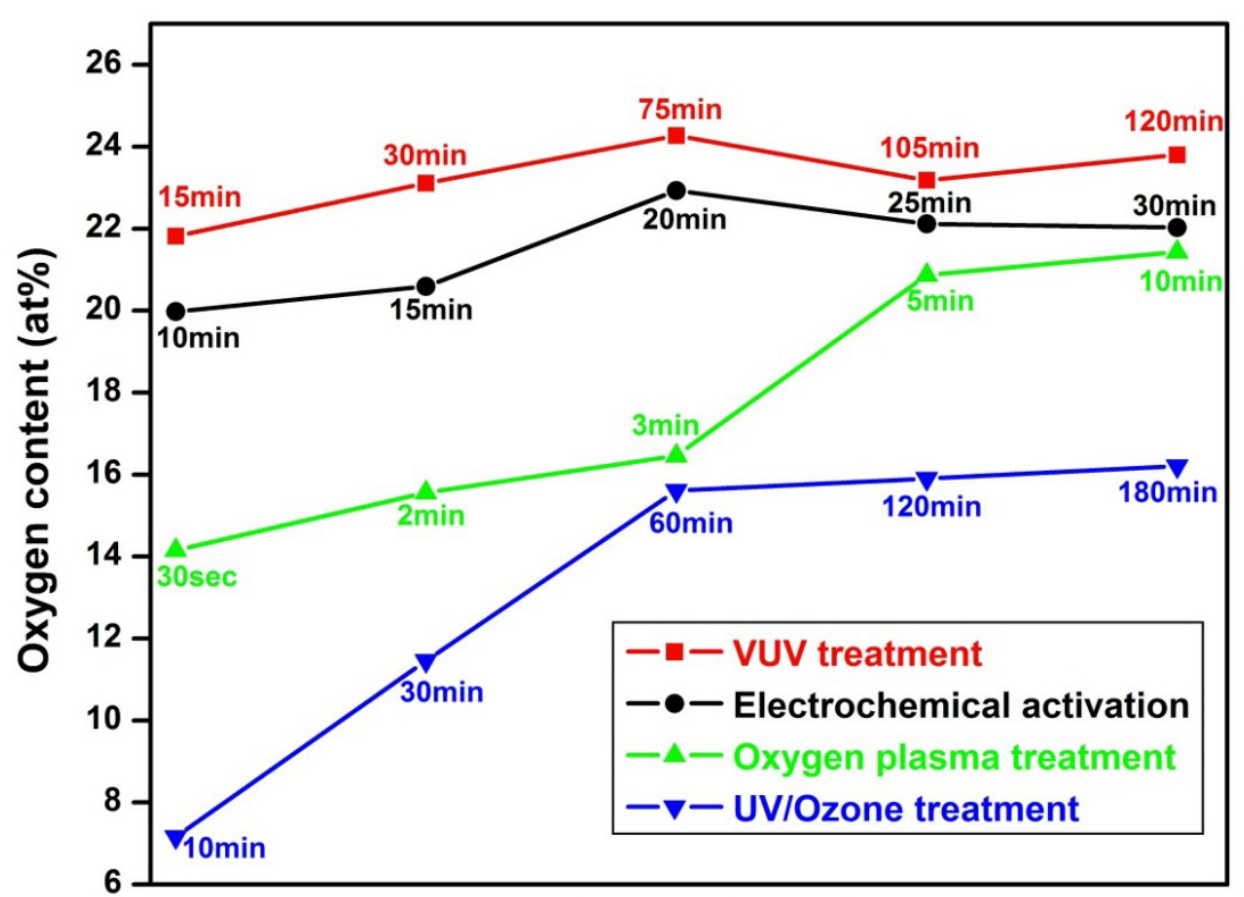

Functionalization time

Figure 6.5. Summary of oxygen concentration (at\%) for different oxidation techniques.

Analysis of the graph shows that the achievable oxidation levels on carbon surface was more than 20 at. $\%$ in the case of VUV pretreatment $(\approx 24$ at.\%), EA pretreatment $(\approx 22$ at.\%), and oxygen RIE pretreatment ( slightly above 20 at.\%). But in the case of UV/ozone pretreatment, only $\approx 15$ at. $\%$ oxygen content was achieved. Thus we conclude that with respect to the achievable oxidation level, VUV-, EA- and oxygen RIE-techniques yield much higher oxygen concentrations compared to UV/ozone pretreatment. However, this does not necessarily indicate different oxidation behavior of the latter technique. It may also be due to a comparatively low UV-intensity, which could yield higher oxygen coverage's but only for impracticably long exposure times. However, as previously mentioned, a significant difference in surface morphology can be 
observed based on the oxidation technique chosen. This signifies the fact that the pretreatment technique should be chosen based on the final application.

Finally, it should be noted that the specified levels of oxygen concentration obtained by XPS apply to a fictitious homogeneous mixture of the constituents (C and O) over the whole information depth of the photoelectrons. In order to obtain information on the chemical groups present on the surface, further investigation of the C1s core level chemical shifts was conducted. Due to the rather broad individual contributions (FWHM typically $1 \mathrm{eV}$ ) the $\mathrm{C} 1 \mathrm{~s}$ peak was decomposed into various components. The following 5 peaks were deconvoluted: $\mathrm{C}-\mathrm{C}\left(\mathrm{sp}^{2}\right)$ at $284.6 \mathrm{eV}, \quad \mathrm{C}-\mathrm{C}\left(\mathrm{sp}^{3}\right)$ at $285.3 \mathrm{eV}, \mathrm{C}^{+\mathrm{I}}(\mathrm{C}-\mathrm{O})$ at $286.2 \mathrm{eV}, \mathrm{C}^{+} \mathrm{II}(\mathrm{C}=\mathrm{O})$ at $287.6 \mathrm{eV}$ and $\mathrm{C}^{+\mathrm{III}}(\mathrm{O}-\mathrm{C}=\mathrm{O})$ at $289.1 \mathrm{eV}$. The primary functional group we focus on in this study is $\mathrm{O}-\mathrm{C}-\mathrm{OH}$ which can be used to bind covalently with amine-terminated biomolecules via amide bonding.

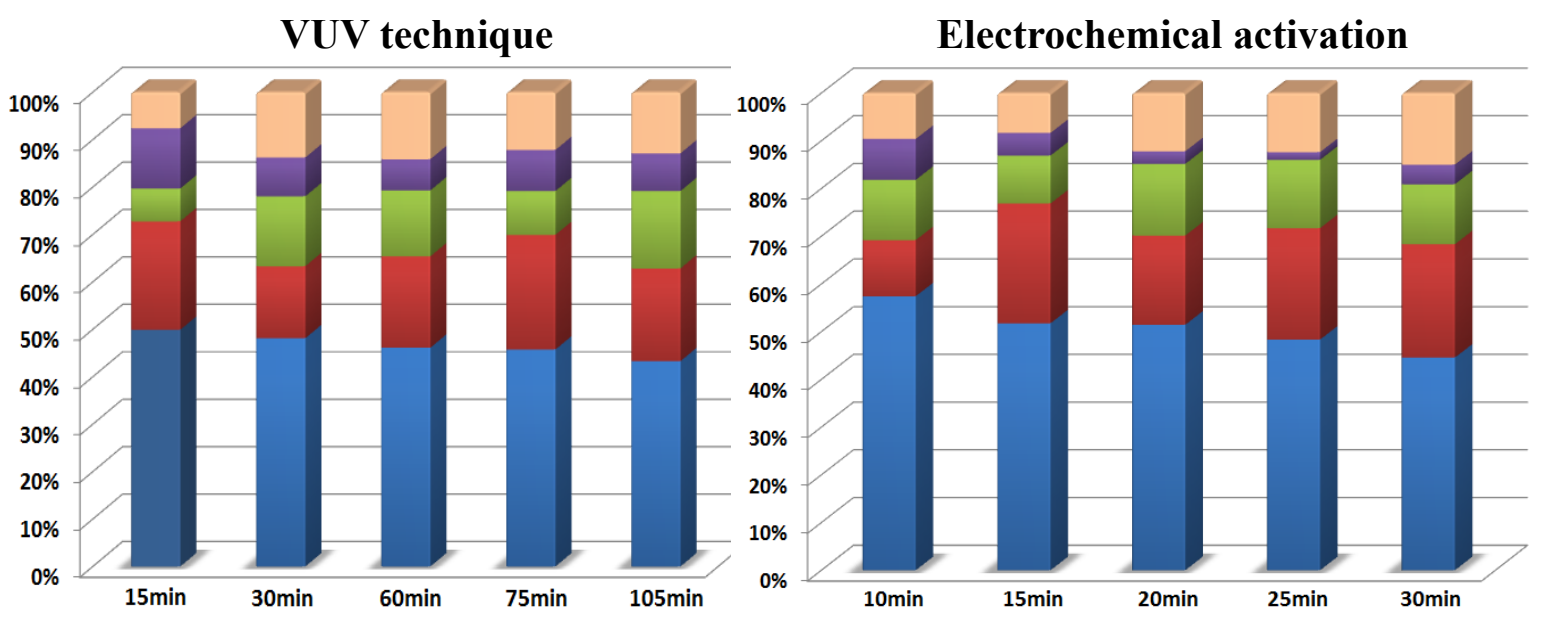



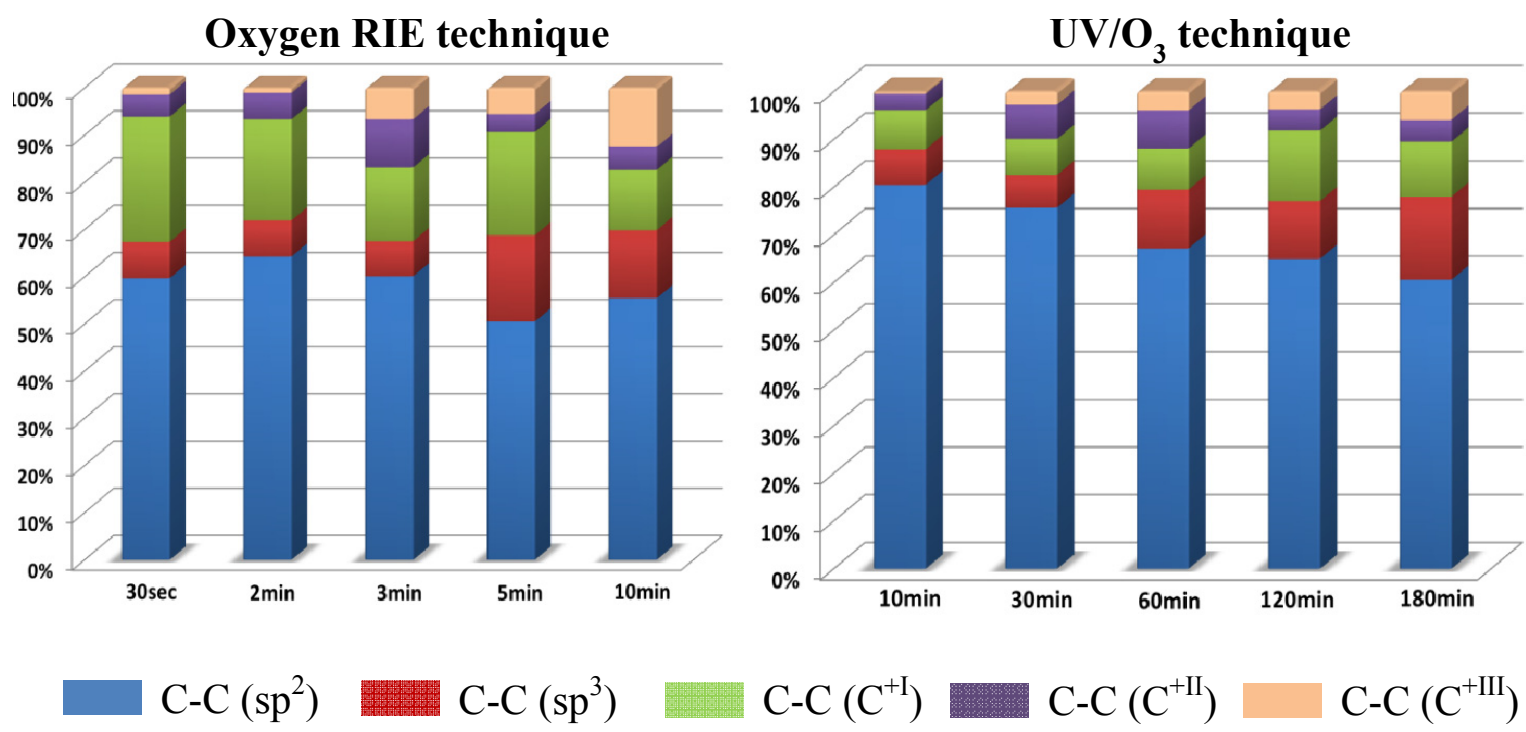

Figure 6.6. Relative contributions of $\mathrm{C}-\mathrm{C}\left(\mathrm{sp}^{2}\right.$ and $\left.\mathrm{sp}^{2}\right), \mathrm{C}^{+\mathrm{I}}, \mathrm{C}^{+\mathrm{II}}$ and $\mathrm{C}^{+\mathrm{III}}$ components as derived from the peak fitting procedure for C-MEMS derived carbon surface oxidized by various techniques.

Figure 6.6 shows the development of the C-C $\left(\mathrm{sp}^{2} \& \mathrm{sp}^{3}\right)$ and the oxygen-related C1s components with increasing oxidation time on the carbon surface. One common trend that can be observed is that upon oxidation the $\mathrm{sp}^{2}$ contents decrease steadily while the oxygen-related components gain in intensity. For all exposures several oxygen-related species are observed with the $\mathrm{C}^{+\mathrm{I}}$ component always being the dominating one. Commonly the $\mathrm{C}^{+}$II component is attributed to carbonyl groups $(\mathrm{C}=\mathrm{O})$. However, in the simplest approximation, when final-state effects in XPS are neglected, the chemical shift is directly related to the oxidation state. Thus $\mathrm{C}^{+\mathrm{II}}$ could as well be caused by chains of bridge-bonded oxygen atoms (i.e. multiple ether groups $-\mathrm{C}-\mathrm{O}-\mathrm{C}-\mathrm{O}-\mathrm{C}-\mathrm{O}-$ ) [12]. Accordingly, rather than signifying a gradual conversion from bridge-bonded etherlike oxygen to on-top "carbonyl" oxygen, the increase of $\mathrm{C}^{+\mathrm{II}}$ may also be interpreted as formation of chains of ether-like groups, as they are favoured by theory $[16,17]$. It was 
observed that in most cases the component $\mathrm{C}^{+\mathrm{III}}$ (representing the carboxyl group) increased with treatment time in all the oxidation techniques. The coverage of the carboxyl group on the surface reached close to $15 \%$ in the case of VUV, $\approx 6 \%$ for $\mathrm{UV} / \mathrm{O}_{3}$ and $\approx 12.5 \%$ for both EA and oxygen RIE. These values are comparable or better than the coverage values obtained for other oxidation pretreatments on the pyrolyzed carbon surface.

\subsection{Conclusions}

Oxidation of C-MEMS derived carbon films were investigated by XPS. All the oxidation techniques except $\mathrm{UV} / \mathrm{O}_{3}$ pretreatment yielded similar oxidation levels. Only $\mathrm{UV} / \mathrm{O}_{3}$ pretreatment yielded somewhat lower values, possibly due to a comparatively low intensity of the UV-source. Compared to EC and oxygen RIE, the VUV and $\mathrm{UV} / \mathrm{O}_{3}$ photochemical pretreatments took more time to reach saturation limit. In all the oxidation techniques, by analysis of the $\mathrm{C} 1 \mathrm{~s}$ core level several coexisting oxygen-containing groups were detected with the carboxyl group increasing with pretreatment time. Finally, this study helps us to choose the oxidation technique for the optimization of functional groups grafted on the surface conducive for covalent binding of bioreceptors.

\subsection{References}

[1] T. Lechleitner, F. Klauser, T. Seppi, J. Lechner, P. Jennings, P. Perco, B. Mayer, D. Steinmüller-Nethl, J. Preiner, P. Hinterdorfer, M. Hermann, E. Bertel, K. Pfaller, W. Pfaller, The surface properties of nanocrystalline diamond and nanoparticulate diamond powder and their suitability as cell growth support surfaces. 2008,Biomaterials, 29, 4275-4284.

[2] F. Maier, J. Ristein, L. Ley, Electron affinity of plasma-hydrogenated and chemically oxidized diamond (100) surfaces. 2001, Physical Review B, 64, 1654111-1654117. 
[3] J.J.B Wilson, J.S. Walton, G. Beamson, Analysis of chemical vapour deposited diamond films by X-ray photoelectron spectroscopy. 2001, J. Electron Spectroscopy and Related Phenomena, 121, 183-201.

[4] K.P. Loh, X.N. Xie, S.W. Yang, J.C. Zheng, Oxygen Adsorption on (111)Oriented Diamond: A Study with Ultraviolet Photoelectron Spectroscopy, Temperature-Programmed Desorption, and Periodic Density Functional Theory. 2002, Journal of Physical Chemistry B, 106, 5230-5240.

[5] P.E. Pehrsson, T.W. Mercer, 2000, Surface Science. 460, 74.

[6] S. Ferro, M. Dal Colle, A. De Battisti, Chemical surface characterization of electrochemically and thermally oxidized boron-doped diamond film electrodes. 2005, Carbon. 43, 1191-1203.

[7] J. Nakamura, T. Ito, Oxidization process of CVD diamond (1 00 ): $\mathrm{H}_{2} \mathrm{X} 1$ surfaces. 2005, Appl. Surface Science, 244, 301-304.

[8] R. Boukherroub, X. Wallert, S. Szunerits, B. Marcus, P. Bouvier, M. Mermoux, Photochemical oxidation of hydrogenated boron-doped diamond surfaces. 1997, Electrochemistry comm., 7, 937-940.

[9] M. Riedel, J. Ristein, L. Ley, The impact of ozone on the surface conductivity of single crystal diamond. 2004, Diamond and related Materials, 13, 746-750.

[10] C.H. Goeting, F. Marken, A. Guitierrez-Sosa, R.G. Compton, J.S. Foord, Electrochemically induced surface modifications of boron-doped diamond electrodes: an X-ray photoelectron spectroscopy study. 2000, Diamond and related Materials, 9, 390-396

[11] H. Notsu, I. Yagi, T. Tatsuma, D.A. Tryk, A. Fujishima, Introduction of oxygencontaining functional groups onto diamond electrode surfaces by oxygen plasma and anodic polarization. 1999, Electrochem. Solid-State Lett., 2, 522-524.

[12] P. Strobel, J. Ristein, L. Ley, Controlled hydroxylation of diamond for covalent attachment of fullerene molecules. 2008, Diamond and related Materials, 17, 13621366.

[13] Katsuyuki Sakuma, Noriyasu Nagai, Naoko Unami, and Shuichi Shoji, Effects of Vacuum Ultraviolet Surface Treatment on the Bonding Interconnections for Flip Chip and 3-D Integration. 2010, IEEE Trans. Electronic Packaging Manufacturing., 33, 212-220. 
[14] A. Bismarck, M.E. Kumru, J. Springer, J. Simitzis, Surface properties of PANbased carbon fibers tuned by anodic oxidation in different alkaline electrolyte systems. 1999, Appl. Surface Science, 143, 45-55.

[15] Three Bond Technical News. 1987, 20, 10pp.

[16] S.J. Sque, R. Jones, P.R. Briddon, Structure, electronics, and interaction of hydrogen and oxygen on diamond surfaces. 2006, Physical Review B, 73, 085313.

[17] M.J. Rutter, J. Robertson, Ab initio calculation of electron affinities of diamond surfaces. 1998, Physical Review B, 57, 9241-9245. 


\section{CHAPTER 7}

\section{DETECTION OF PLATELET-DERIVED GROWTH-FACTOR (PDGF) USING SIGNALING APTAMER/ PROTEIN BINDING COMPLEX}

\subsection{Introduction}

With the increasing application of proteomic strategies for the detection of cancer related oncoproteins and discovery of biomarkers, it is of extreme interest to develop portable platforms for sensitive detection of proteins and their molecular variants. Aptamers are single stranded DNA or RNA molecules selected in vitro from DNA/ RNA random pools that are capable of binding with biological entities such as proteins, cells along with small molecules, drugs, peptides and hormones with high affinity and specificity [1-3]. Aptamers have been sought out as ideal alternative candidates to the traditional antibodies for use in analytical devices due to their easy synthesis, high binding affinity, long storage times, and excellent selectivity [4]. Recent studies have demonstrated the applicability of aptamers to target a disease state, such as cancer [5]. This opens up new avenues in the future for aptamers to potentially substitute more established components for therapeutics and/or diagnostics.

Platelet-derived growth factor (PDGF) is a protein that regulates cell growth and division. Overexpression of PDGF has been associated with several human health disorders including atherosclerosis (hardening of the arteries) [6], balloon injury induced restenosis (narrowing of blood vessels) [7], pulmonary hypertension [8], organ fibrosis (formation of excess fibrous connective tissue in an organ or tissue) [9], tumorigenesis (formation of tumors) [10]. PDGF receptors are almost undetectable in normal vessels, but are highly expressed in the diseased vessels. A PDGF dimer composed of two 
different types of monomer (A and B chains) occurs in three variants: PDGF-BB, PDGF$\mathrm{AB}$ and PDGF-AA. In particular, oncoprotein PDGF-BB is often overexpressed in human malignant tumors and known as a potential protein marker for cancer diagnosis [10].

In recent years, PDGF-BB protein detection using fluorescence [11-20], colorimetry [21] and electrochemistry techniques have been reported [22-24]. These methods involve either labeling the aptamer with a fluorophore, or the use of redox species. In fluorescence based PDGF detection techniques, fluorophore-labeled aptamers are used to signal binding by monitoring the changes of fluorescence intensity [14] or anisotropy resulting from the changes of the microenvironment [13] or rotational motion through fluorescence energy transfer [15]. However, as the precise target binding sites and the conformational changes of the aptamers are generally unknown, it is not easy to design labeling strategies [19]. Besides, there is always a concern that the conjugation of a fluorophore to an aptamer will ultimately weaken the affinity of the aptamer to its ligand [19]. In the case of electrochemistry based detection techniques, due to the use of redox species, the electrodes are limited to conductive materials and also the different linkers used to attach the aptamer onto the electrode surface (such as gold) exhibits rapid degradation with time [25]. Most recently, diamond substrate has been used to detect PDGF by monitoring the fluorescence change from the release of an intercalating dye when the probe aptamer captures the target [26]. Although the sensor showed good sensitivity and selectivity, the use of diamond substrates is not cost effective. The controllability of defects and grain boundaries in polycrystalline diamond substrates 
along with the high operating cost due to the need for high vacuum and high temperature systems are limiting factors for mass production.

Traditionally for biological and electrochemical sensing, glassy carbon is one of the popularly used materials due to its low cost, better resistance towards biofouling, biocompatibility, good electrical conductivity, low background capacitance, and the flexibility to tailor the surface by various physical/chemical treatments. In particular, carbon synthesized by carbon-microelectromechanical systems (C-MEMS) technique is intriguing since it exhibits reaction kinetics comparable to glassy carbon, but with lower oxygen/carbon atomic $(\mathrm{O} / \mathrm{C})$ ratio [27-29]. Since photolithography technique is used for patterning purpose, the electrodes obtained by this manner have better resolution and reproducibility compared to screen printed carbon paste electrodes. C-MEMS technique is actively pursued to fabricate electrodes for energy storage/ conversion devices, electrochemical sensors and biodetection devices [30-34] due to the versatility in the experimental approach to produce high surface area 3D carbon microarrays. In addition, our group has already demonstrated the ability to tailor the carbon surface by introducing nanoporosity using a block copolymer as porogen [35] and integration of functional nanomaterials such as graphene [36] and carbon nanotubes [34] on the surface of 3D carbon microarrays. The high surface area of the 3D carbon microarrays makes it an ideal platform for increased biomolecule loading to improve the sensitivity and performance of the functional devices.

In this chapter, a signaling aptamer/protein binding complex on 3D carbon micropillar arrays using TOTO intercalating dye to signal PDGF-BB-aptamer binding was reported. The carbon surface was functionalized by direct amination technique to 
introduce amino groups for covalent immobilization of target binding aptamer. It was demonstrated that this simple detection technique offered high sensitivity with PDGF detection in the sub-nanomolar range and good selectivity against different proteins, which could be extended for the detection of other biomarker proteins.

\subsection{Materials and Methods}

The 5'-carboxyl-modified PDGF-B-binding aptamer (5 - CAG GCT ACG GCA CGT AGA GCA TCA CCA TGA TCC TG-3’), PDGF-BB, PDGF-AB, PDGF-AA, adenosine triphosphate (ATP), and calmodulin were purchased from Sigma Genosys, Japan. The intercalating dye 1,1-(4,4,8,8-tetramethyl- 4,8-diazaundecamethylene)-bis-4[3-methyl-2,3-dihydro(benzo-1,3-thiazole)-2-methylidene] quinolinium tetraiodide (TOTO) was purchased from Invitrogen Corporation, USA. N-hydroxysuccinimide (NHS) and 1-ethyl-3-(3-dimethylaminopropyl) carbodiimide hydrochloride (EDC) were purchased from Sigma Aldrich, Japan. NANO ${ }^{\mathrm{TM}}$ SU-8 and NANO ${ }^{\mathrm{TM}}$ developer were obtained from Microchem, USA.

\subsubsection{Fabrication of 3D Carbon Micropillar Arrays}

The three-dimensional carbon microarrays were fabricated by a typical C-MEMS process. 4 in. silicon oxide wafers were spin cleaned and NANO ${ }^{\text {TM }}$ SU-8 100 negative photoresist was spin coated at $500 \mathrm{rpm}$ for $12 \mathrm{sec}$ and then $1200 \mathrm{rpm}$ for $30 \mathrm{sec}$. The final thickness of the film was approximately $200 \mu \mathrm{m}$ photoresist film. The photoresist was baked at $65^{\circ} \mathrm{C}$ for $10 \mathrm{~min}$ and at $95^{\circ} \mathrm{C}$ for $30 \mathrm{~min}$. The photoresist was patterned by exposure using OAI Hybralign contact aligner (light intensity, $17 \mathrm{~mW} / \mathrm{cm}^{2}$ ) for $60 \mathrm{sec}$. Post expose bake was carried out at temperatures of $65^{\circ} \mathrm{C}$ for $1 \mathrm{~min}$ and $95^{\circ} \mathrm{C}$ for $3 \mathrm{~min}$ followed by developing using NANO ${ }^{\mathrm{TM}} \mathrm{SU}-8$ developer (Microchem, USA) for $15 \mathrm{~min}$. 
The pyrolysis of the photoresist microarrays was conducted in a tube furnace under $(95 \%$ $\mathrm{N}_{2}+5 \% \mathrm{H}_{2}$ ) environment. The samples were heated from room temperature to $350{ }^{\circ} \mathrm{C}$ at $2{ }^{\circ} \mathrm{C} / \mathrm{min}$ rate with a hold time of $40 \mathrm{~min}$, followed by ramping to $1000{ }^{\circ} \mathrm{C}$ at $5{ }^{\circ} \mathrm{C} / \mathrm{min}$ rate and hold time of $60 \mathrm{~min}>$ the samples were cooled down to room temperature in the inert atmosphere.

\subsubsection{Surface Functionalization}

Before the direct amination process, the samples were first thoroughly rinsed with DI water and blow dried. The amination process was performed at room temperature in an ammonia gas (99.9\%) environment and using UV lamp $(\lambda=253.7 \mathrm{~nm})$. Prior to UV irradiation, the reaction chamber was purged with nitrogen gas for 5 min to remove oxygen and other gases. The reaction chamber was then irradiated with UV light for $4 \mathrm{hr}$ under a continuous flow of ammonia gas at $100 \mathrm{sccm}$. Finally, nitrogen gas is purged for 5 min to remove any ammonia in the reaction chamber before removing the sample. A detailed schematic showing the direct amination process is shown in Chapter 5, Section 5.2.2.

\subsubsection{PDGF Detection}

The carboxyl modified aptamer was covalently immobilized on the aminoterminated carbon surface without the use of any linker molecules. The probe aptamer with $3 \times$ sodium saline citrate (SSC) buffer solution, $0.1 \mathrm{M}$ N-hydroxysuccinimide (NHS) and 0.4 M 1-ethyl-3-(3-dimethylaminopropyl) carbodiimide hydrochloride (EDC) were mixed in a 2:1:1 ratio. The final concentration of the probe aptamer solution was $20 \mu \mathrm{M}$. $5 \mu \mathrm{l}$ of the probe aptamer solution was dropped onto the 3D carbon microarrays and incubated for $2 \mathrm{~h}$ at $38{ }^{\circ} \mathrm{C}$ in a humidified chamber. After immobilization, the sample was 
washed in PBS+ Tween-20 (PBS: $1 \mathrm{mM} \mathrm{NaCl}: 2 \mathrm{mM} \mathrm{NaH}_{2} \mathrm{PO}_{4}: 8 \mathrm{mM} \mathrm{Na}_{2} \mathrm{HPO}_{4} ; 0.1 \%$ Tween-20) solution for $5 \mathrm{~min}$ and three times with deionized (DI) water for 3 min each The probe aptamer was then reacted with $10 \mu \mathrm{M}$ intercalating dye (TOTO) diluted in TE buffer $[10 \mathrm{mM}$ tris (hydroxymethyl)- aminomethane $\quad$ (Tris), $1 \mathrm{mM}$ ethylenediaminetetraacetic acid (EDTA), $\mathrm{pH} \sim 8]$ for $1 \mathrm{~h}$ at $25^{\circ} \mathrm{C}$. Following the intercalation of the dye, the sample was cleaned by TE buffer for 20 min and a DI water rinse. PDGF-BB protein diluted in $2 \times \mathrm{SSC}$ was then bound to the immobilized aptamer at room temperature for $1 \mathrm{~h}$ at $25{ }^{\circ} \mathrm{C}$. Unbound PDGF-BB were cleaned by DI water for 5 min. It is noteworthy that certain monovalent and divalent cations commonly encountered in biological specimens are known to affect DNA conformation. For this reason, the concentrations of the solution based on our previous study concerning the effect of protein binding based on $\mathrm{Mg}^{2+}$ cation and $\mathrm{NaCl}$ concentration in PBS buffer solution [28]. Finally, in order to regenerate the sensor by dissociating PDGF-BB and intercalator from the probe aptamer, the sample is washed in $10 \%$ sodium dodecyl sulfate (SDS) solution for $30 \mathrm{~min}$.

\subsubsection{Characterization}

The morphology of 3D carbon microarrays was investigated using JOEL 6335 FE- SEM scanning electron microscopy. Raman spectrum was collected with an argon ion laser system (Spectra Physics, model 177G02) of $\lambda=514.5 \mathrm{~nm}$ at a laser power of ca. $7 \mathrm{~mW}$. The chemical composition of pyrolyzed photoresist carbon film before and after d1irect amination procedure was investigated by an Ulvac $\Phi 3300$ x-ray photoelectron spectroscopy (XPS) with an anode source providing $\mathrm{Al} \mathrm{K \alpha}$ radiation. The 
electron takeoff angle was $45 \pm 3^{\circ}$ relative to the substrate surface. Fluorescence observation was performed using an Olympus IX71 epifluorescence microscope.

\subsection{Results and Discussion}

\subsubsection{Characterization}

A typical SEM image of high aspect ratio 3D carbon micropillar arrays is shown in Figure 8.1a. The average dimensions of the carbon micropillars after carbonizing patterned SU-8 photoresist structures are $\sim 160 \mu \mathrm{m}$ height and $\sim 30 \mu \mathrm{m}$ width. A careful examination of the SEM image shows that the upper half and especially the top part of the carbon micropillars is slightly wider compared to the lower half. This could be could due to the higher dose of UV light experienced by the top layer of the thick photoresist [27].Raman spectroscopy was used to investigate the crystallinity of the carbon micropillars. Figure $8.1 \mathrm{~b}$ shows the Raman spectrum of pyrolyzed carbon with two significant broad peaks at $\sim 1350 \mathrm{~cm}^{-1}$ (D-band) and $\sim 1590 \mathrm{~cm}^{-1}$ (G-band). The first peak at $1350 \mathrm{~cm}^{-1}$ represents the disorder band of the microcrystallite graphite and the second peak at $1590 \mathrm{~cm}^{-1}$ is due to the single Raman line typically found on single crystalline graphite. The $\mathrm{I}_{\mathrm{D}} / \mathrm{I}_{\mathrm{G}}$ ratio of 1.1 indicates that carbon obtained from pyrolysis of photoresist is identical to glassy carbon synthesized at same temperature [37]. 

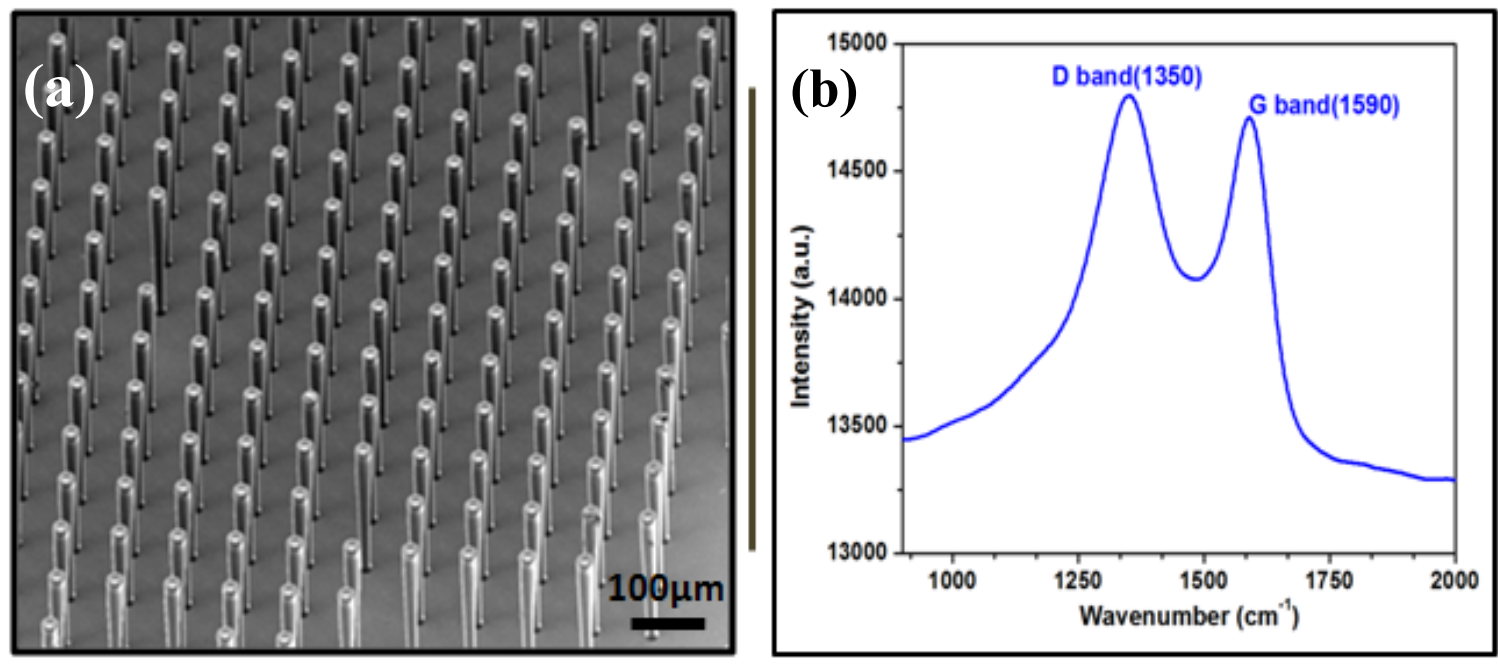

Figure 7.1 (a) Typical SEM image of 3D carbon microarrays, (b) Raman spectrum of pyrolyzed photoresist film showing the two prominent bands at 1350 and $1590 \mathrm{~cm}^{-1}$.

\subsubsection{Surface Functionalization}

It is well documented that the termination or functionalization of the surface is one of the key issues in the interaction and immobilization of biomolecules [39]. In this work, to covalently immobilize PDGF binding aptamer on the carbon surface, the sample was first treated by direct amination technique [30] where the sample was irradiated by ultraviolet (UV) light $(\lambda=253.7 \mathrm{~nm})$ in an ammonia gas environment for $4 \mathrm{hrs}$. In contrast to oxidation techniques which introduce several oxygen-based functional groups such as ketone, hydroxyl, and carboxyl groups, only $\mathrm{NH}_{2}$ bonds are expected to form on the carbon surface by direct amination procedure due to their chemical structure. The elemental composition and surface binding of pyrolyzed photoresist film were evaluated by X-ray photoelectron spectroscopy spectra (XPS) as shown in Figure 8.2. Analysis of the widescan XPS spectra of the bare carbon film before amination (Fig 2 inset) shows two major peaks evident of carbon $(284.6 \mathrm{eV})$ and oxygen $(531.8 \mathrm{eV})$ but in the case of after amination, three distinct peaks representing carbon, oxygen and nitrogen $(398.4 \mathrm{eV})$ 
are evident. The nitrogen peak visible after amination is a result of ammonia gas forming $\mathrm{C}-\mathrm{NH}_{2}$ on the carbon substrate [30]. The deconvoluted high resolution C1s spectrum (Fig 2) shows major carbon peaks at $284.6 \mathrm{eV}\left(\mathrm{sp}^{2}\right)$ and $285.2 \mathrm{eV}\left(\mathrm{sp}^{3}\right)$, respectively. The other peaks at $285.4 \mathrm{eV}, 286.3 \mathrm{eV}, 287.6 \mathrm{eV}$ and $289.1 \mathrm{eV}$ corresponds to $\mathrm{C}-\mathrm{N}, \mathrm{C}-\mathrm{O}$, $\mathrm{C}=\mathrm{O}$ and $\mathrm{O}-\mathrm{C}=\mathrm{O}$ bonds, respectively. The maximum surface coverage of amino groups achieved was $\sim 8 \%$, which is similar to the amino coverage previously reported [30].

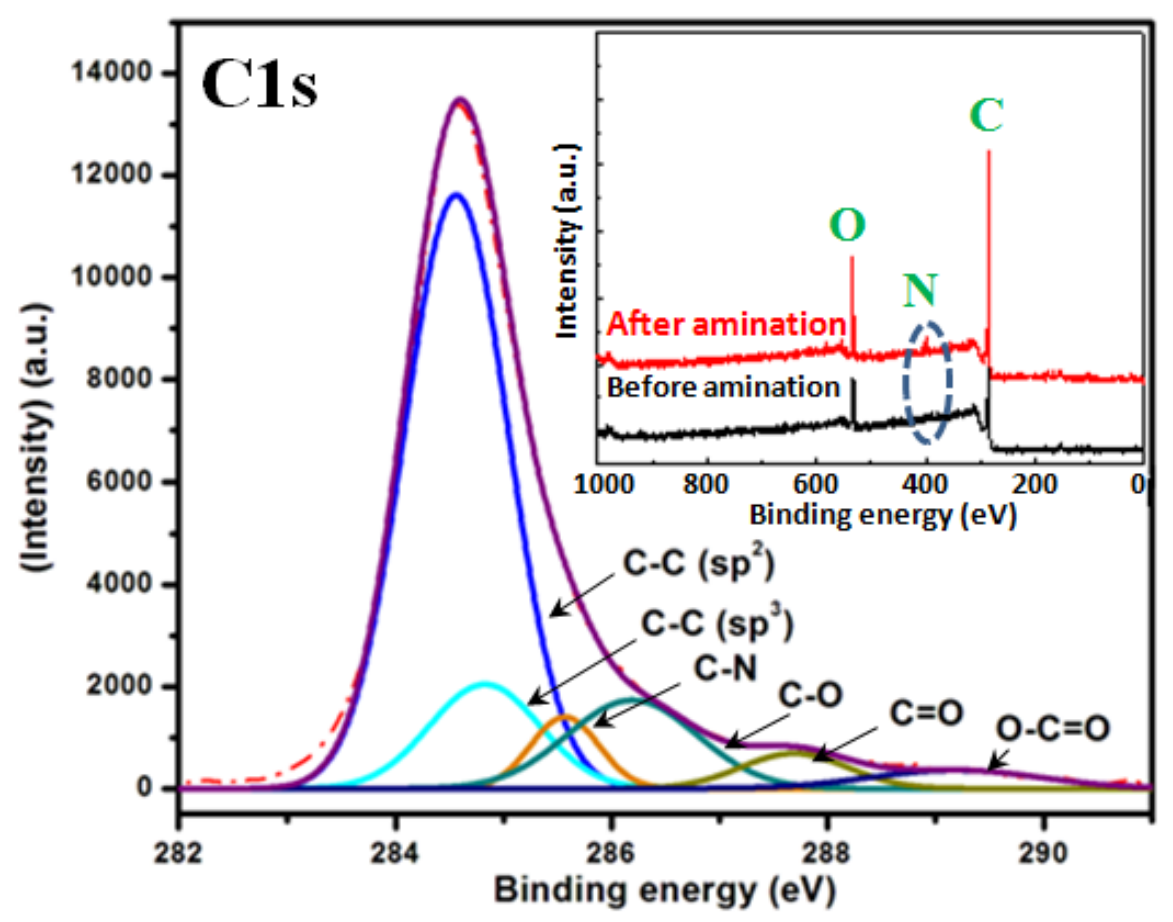

Figure 7.2. Deconvoluted C1s spectra of pyrolyzed photoresist film after $4 \mathrm{hr}$ direct amination, here dash line shows the original data and solid lines show the fitting curves. Inset shows the widescan XPS spectra of carbon film before and after amination.

\subsubsection{Signaling Aptamer/ Protein Binding Complex Mechanism}

The detection of PDGF-BB using signaling aptamer/protein binding complex strategy is shown schematically in Figure 8.3. (I) The carboxyl-terminated PDGF-binding aptamer (probe aptamer) is first covalently attached to the amine-terminated carbon 
surface via amide binding. (II) Subsequently the TOTO dye was intercalated with PDGFbinding aptamer. The TOTO dye shows no fluorescence in aqueous solution but exhibits strong fluorescence when bound to the nonaqueous pocket of the duplex nucleic acid regions in the aptamer. It is important to note that the fluorescence signal from TOTO is dependent on its local environment and DNA/RNA conformation. (III) When the target PDGF-BB protein bonds with the aptamer, the induced conformational change of the aptamer, as well as the blocking of intercalated TOTO dye results in a significant proteindependent fluorescence change. (IV) Finally for regenerating the sensor, the aptamer intercalating dye complex and PDGF-BB are dissociated by treatment with sodium dodecyl sulfate (SDS).

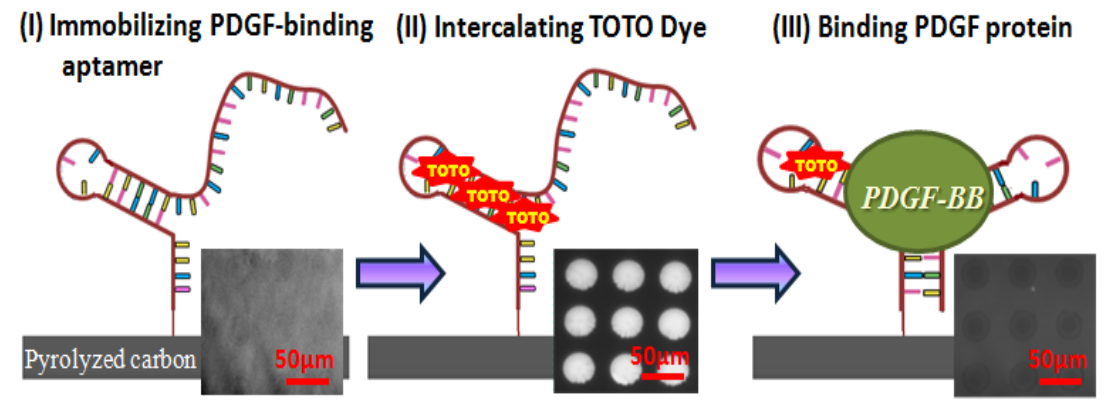

(IV) Regenerating PDGF sensor

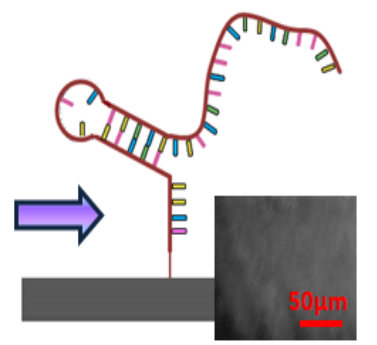

Figure 7.3. Schematic illustration of the detection of PDGF-BB using signaling aptamer/protein binding complex on 3D carbon microarrays platform; (I) covalent immobilization of PDGFbinding aptamer on partially aminated carbon surface, (II) intercalating the probe aptamer with TOTO fluorescent dye, (III) binding PDGF-BB to the aptamer-intercalating dye complex, (IV) regenerating the sensor by sodium dodecyl sulfate (SDS) treatment to remove PDGF and release the intercalating dye. 


\subsubsection{Sensitivity and Selectivity of the Sensor}

The relationship of the change in the relative fluorescence difference with different concentrations of PDGF-BB in 2xSSC (saline-sodium citrate) solution was evaluated to study the sensitivity of the sensor, as shown in Figure 8.4. At first, the difference in the fluorescence intensities is computed from the fluorescence intensity values obtained after initial TOTO intercalation with the probe aptamer and then after PDGF-BB binding with the probe aptamer. Finally, the relative fluorescence difference is calculated by dividing the value obtained from difference in fluorescence intensities and initial fluorescence intensity. As expected, analysis of the data shows that the relative fluorescence difference increased as the concentration of PDGF-BB was increased from $0.005-100$ nmol. This can be explained by the fact that, as the PDGF-BB concentration is increased, more intercalator dye is released from the aptamer which results in a larger difference in the relative fluorescence. A near linear relationship between the relative fluorescence difference and the protein concentration was observed even in the sub-nanomolar range. A low detection limit of $0.005 \mathrm{nmol}$ was achieved, and indicates that the sensor detection limit is much below the typical detection range of the PDGF in clinical samples. The detection limit by other reported aptamer-based analytical techniques, for example, is 1 $\mathrm{nmol}$ in undiluted serum and $0.05 \mathrm{nmol}$ in 50\% serum was achieved with electrochemical detection [22], $0.1 \mathrm{nmol}$ using solution based fluorescent signaling complex of aptamer and TOTO [14], and 2 nmol with fluorescence anisotropy based detection [13]. Typical PDGF concentrations of normal individuals and cancer patients have been found to be in the sub-nanomolar range: $0.4-0.7 \mathrm{nmol}$ in human blood serum and $0.008-0.04 \mathrm{nmol}$ in 
human plasma [12]. Therefore, with the excellent sensitivity achieved, this PDGF sensor has the potential to be used in clinical setting.

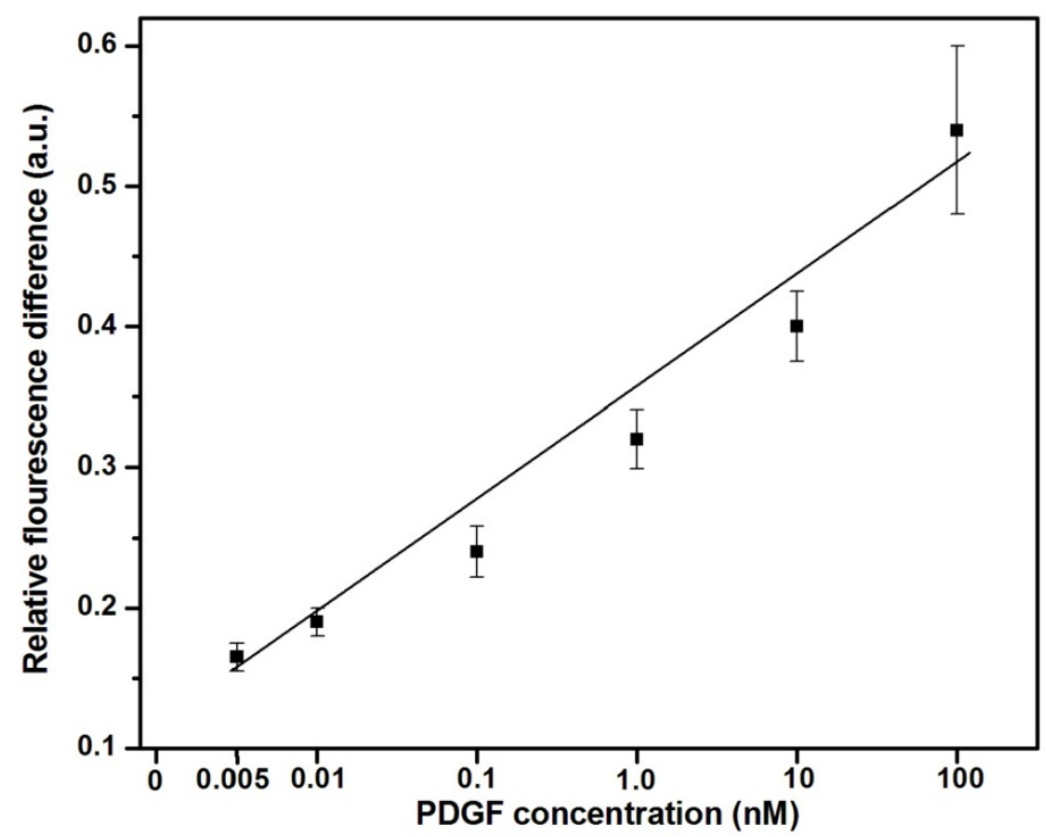

Figure 7.4: Relative fluorescence difference response of the sensor to different concentrations of PDGF from $0.005 \mathrm{nM}$ to $100 \mathrm{nM}$. The concentrations of the aptamer and intercalating dye were $20 \mu \mathrm{M}$ and $10 \mu \mathrm{M}$, respectively.

After the regeneration of the same sensor platform, in order to detect the PDGF using the aptamer based sensor, the probe should selectively respond to PDGF-BB, free or distinguishable from the interference by other biological components. Figure 8.5 shows the selectivity test of PDGF binding aptamer towards the three variants of PDGF along with bovine serum albumin, calmodulin, and ATP, which are all typically present in the blood. The graph shows that the relative fluorescence difference for PDGF-BB binding with probe aptamer was about two times that of PDGF-AB and 10-times that of PDGF-AA binding with the same probe aptamer, respectively. Further, fluorescence 
intensity difference for other biomolecules such as bovine serum album (BSA), ATP and calmodulin was approximately 70 fold smaller when compared to the value obtained for PDGF-BB binding. These results could be explained mainly by the fact that the PDGFbinding aptamer used in this work binds to the three isoforms of PDGF (PDGF-BB, PDGF-AB, and PDGF-AA) with different affinities. Since the target binding aptamer has high specificity toward PDGF-BB, the corresponding reduction in the fluorescence intensity caused by PDGF-AA was clearly lower due to the absence of any binding sites on the aptamer towards PDGF-AA. On the other hand, PDGF-AB protein consists of both $\mathrm{A}$ and $\mathrm{B}$ chains meaning only one site that could bind to the aptamer. The amino acid sequences of PDGF-A is $60 \%$ similar to that of PDGF-B. Therefore, this sensor can detect isoforms with good selectivity. In the other cases where different biomolecules such as BSA, ATP and calmodulin are introduced towards the target binding aptamer, no significant binding is expected due to the unavailability of the binding site and therefore no major relative fluorescence difference was detected. It is noteworthy that although BSA usually contains a high concentration of proteins, it does not affect the selectivity of the probe aptamer used. The excellent selectivity of the sensing platform achieved in this work exhibits the promise of aptamers for cancer biomarker detection. The sensitivity and selectivity of our sensor platform could be even further improved when using high surface area 3D carbon microarrays integrating with functional nanomaterials such as graphene [36] and carbon nanotubes [34]. 


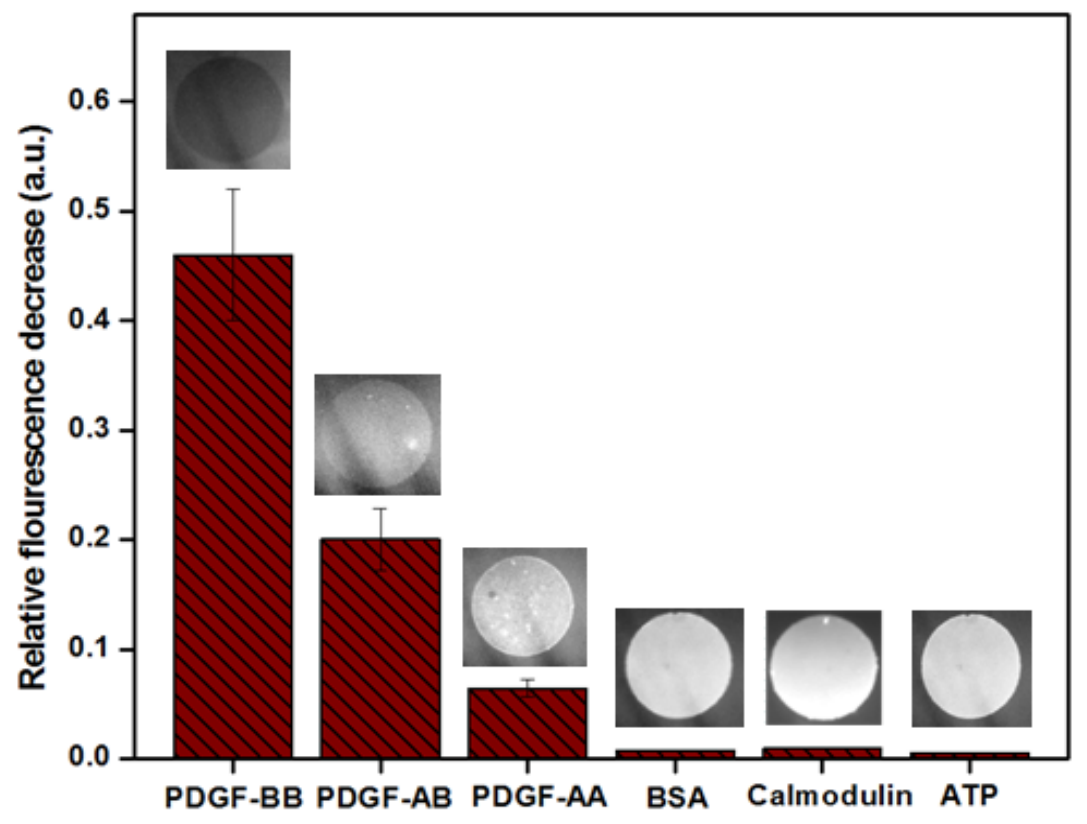

Figure 7.5. Comparison of relative fluorescence difference of different proteins towards PDGF binding aptamer ; The concentration of the different molecules (PDGF-BB, PDGF-AB, PDGFAA, BSA, ATP and calmodulin) was $100 \mathrm{nM}$ and concentrations of PDGF-binding aptamer and intercalating dye were $20 \mu \mathrm{M}$ and $10 \mu \mathrm{M}$, respectively.

\subsection{Conclusions}

In summary, high sensitive detection of PDGF using aptamer/protein binding complex on the 3D carbon microarray platform was achieved. For covalent immobilization of the probe aptamer, the carbon surface was bio-functionalized using direct amination technique. The sensor showed a near linear relationship towards protein concentration even in the sub-nanomolar range with excellent selectivity towards other biomolecules. The robust platform of signaling aptamer/protein binding complex on 3D carbon microarrays has the ability to detect wide variety of biomarkers and proteins for potential application in the preliminary diagnosis of cancer. 


\subsection{References}

[1] A. D. Ellington, J. W. Szostak, In vitro selection of RNA molecules that bind specific ligands. 1990, Nature, 346, 818-822.

[2] D. L. Robertson, G.F. Joyce, Selection in vitro of an RNA enzyme that specifically cleaves single-stranded DNA. 1990, Nature, 344, 467-468.

[3] C. Tuerk, L. Gold, Systematic evolution of ligands by exponential enrichment: RNA ligands to bacteriophage T4 DNA polymerase. 1990, Science, 249, 505-510.

[4] S.D. Jayasena, Aptamers: An emerging class of molecules that rival antibodies in diagnostics. 1999, Clin Chem., 45,1628-1650.

[5] D Shangguan, Y Li, Z Tang, Z. C. Cao, H.W. Chen, P. Mallikaratchy, K. Sefah, C. J. Yang, W.Tan, Aptamers evolved from live cells as effective molecular probes for cancer study. 2006, Proc. Natl. Acad. Sci. USA, 103, 11838-11843.

[6] M. Lassila, T. J. Allen, Z. Cao, V. Thallas, K. A. Jandeleit-Dahm, R. Candido, M. E. Cooper, Imatinib Attenuates Diabetes-Associated Atherosclerosis. 2004, Arterioscler Thromb Vasc Biol, 24, 935-942.

[7] A. Szabó, J. Laki, H. O. Madsen, E. Dósa, Z. Prohászka, S. Rugonfalvi-Kiss, M. Kókai, G. Acsádi, I. Karádi, L. Entz, L. Selmeci, L. Romics, G. Füst, P. Garred, Early rise in Serum VEGF and PDGF Levels Predisposes Patients With a normal MBL2 Genotype to restenosis after eversion endarterectomy. 2007, Stroke, 38, 2247-2253.

[8] R. J. Barst, PDGF signaling in pulmonary arterial hypertension. 2005, J. Clin. Invest., 115, 2691-2694.

[9] M. Trojanowska, Role of PDGF in fibrotic diseases and systemic sclerosis. 2008, Rheumatology, 47, v2-v4.

[10] A.H. Shih, C. Dai, X. Hu, M. K. Rosenblum, J. A. Koutcher, E. C. Holland, Dosedependent effects of platelet-derived growth factor-B on glial tumorigenesis. 2004, Cancer Res., 64, 4783-4789.

[11] L. Yang, C. W. Fung, E. J. Cho, A. D. Ellington, Real-time rolling circle amplification for protein detection. 2007, Anal. Chem., 79, 3320-3329.

[12] A. R. Ruslinda, V. Penmatsa, Y. Ishii, S. Tajima, H. Kawarada, Highly sensitive detection of platelet-derived growth factor on a functionalized diamond surface using aptamer sandwich design. 2012, Analyst, 137,1692-1697. 
[13] X. Fang, Z. Cao, T. Beck, W. Tan, Molecular Aptamer for Real-Time Oncoprotein Platelet-Derived Growth Factor Monitoring by Fluorescence Anisotropy. 2001, Anal. Chem., 73, 5752-5757.

[14] X. Fang, A. Sen, M. Vicens, W. Tan, Synthetic DNA Aptamers to detect protein molecular variants in a high throughput fluorescence quenching assay. 2003, ChemBioChem, 4, 829-834.

[15] M. C. Vicens, A. Sen, A. Vanderlaan, T. J. Drake, W. Tan, Investigation of molecular beacon aptamer-based bioassay for platelet-derived growth factor detection. 2005, ChemBioChem, 6, $900-907$.

[16] C. J. Yang, S. Jockusch, M. Vicens, N. J. Turro, W. Tan, Light-switching excimer probes for rapid protein monitoring in complex biological fluids. 2005, Proc. Natl. Acad. Sci. USA, 102, 17278-17283.

[17] Y. Jiang, X. Fang, C. Bai, Signaling aptamer/protein binding by a molecular light-switch complex. 2004, Anal. Chem., 76, 5230-5235.

[18] C. Zhou, Y. Jiang, S. Hou, B. Ma, X. Fang, M. Li, Detection of oncoprotein platelet-derived growth factor using a fluorescent signaling complex of an aptamer and TOTO. 2006, Anal. Bioanal. Chem., 384, 1175-1180.

[19] C. Huang, S. Chiu, Y. Huang, H. Chang, Aptamer-functionalized gold nanoparticles for turn-on light switch detection of platelet-derived growth factor. 2007, Anal. Chem., 79, 4798-4804.

[20] C. Huang, C. Chiang, Z. Lin, K. Lee, H. Chang, Bioconjugated gold nanodots and nanoparticles for protein assays based on photoluminescence quenching. 2008, Anal. Chem., 80, 1497-1504.

[21] C. Huang, Y. Huang, Z. Cao, W. Tan, H. Chang, Aptamer-modified gold nanoparticles for colormetric determination of platelet-derived growth factors and their receptors. 2005, Anal. Chem., 77, 5735-5741.

[22] R. Y. Lai, K. W. Plaxco, A. J. Heeger, Aptamer-Based Electrochemical Detection of Picomolar Platelet-Derived Growth Factor Directly in Blood Serum. 2007, Anal. Chem., 79, 229-233.

[23] T. H. Degefa, J. Kwak, Label-free aptasensor for platelet-derived growth factor (PDGF) protein. 2008, Anal. Chim. Acta, 613, 163-168.

[24] A. R. Ruslinda, S. Tajima, Y. Ishii, Y. Ishiyama, R. Edgington, H. Kawarada, Aptamer-based biosensor for sensitive PDGF detection using diamond transistor. 2010, Biosens. and Bioelec., 26, 1599-1604. 
[25] M. F. Phillips, M. R. Lockett, M. J. Rodesch, M. R. Shortreed, F. Cerrina, L. M. Smith, In situ oligonucleotide synthesis on carbon materials: stable substrates for microarray fabrication. 2008, Nucleic Acids Res., 36, e7.

[26] Y. Ishii, S. Tajima, H. Kawarada, Aptasensor for oncoprotein platelet-derived growth factor detection on functionalized diamond surface by signal-off optical method. 2011, Appl. Phys. Exp., 4, 027001-3.

[27] C. Wang, L. Taherabadi, M.Madou, A novel method for the fabrication of highaspect ratio C-MEMS structures. 2005, IEEE J. Microelectromechanical Systems, 14, 348-358.

[28] S. Ranganathan, R. McCreery, S.M. Majji, M. Madou, Photoresist-Derived Carbon for Microelectromechanical Systems and Electrochemical Applications. 2000, J. Electrochem. Soc, 147, 277-282.

[29] A. Singh, J. Jayaram, M. Madou, S. Akbara. Pyrolysis of negative photoresists to fabricate carbon structures for microelectromechanical systems and electrochemical applications. 2002, Journal of the Electrochemical Society, 149, E78-E83.

[30] J.H. Yang, V. Penmatsa, S. Tajima, H. Kawarada, C. Wang, Direct amination on 3-dimensional pyrolyzed carbon micropattern surface for DNA detection. 2009, Materials Letters, 63, 2680-2683.

[31] H. Xu, K. Malladi, C. Wang, L. Kulinsky, M. Song, M. Madou, Carbon postmicroarrays for glucose sensors. 2008, 23, 163-1644.

[32] J. A. Lee, S. Hwang, J. Kwak, S. I. Park, S. S. Lee, K-C Lee, An electrochemical impedance biosensor with aptamer-modified pyrolyzed carbon electrode for labelfree protein detection. 2008, Sensors and Actuators B, 129, 372-379.

[33] C. Wang, L. Taherabadi, G. Jia, M. Madou, Y. Yeh, B. Dunn, C-MEMS for the manufacture of $3 D$ microbatteries. 2004, Electrochemical and Solid-State Letters, 7, A435- A438.

[34] W. Chen, M. Beidaghi, V. Penmatsa, K. Bechtold, L. Kumari, W.Z. Li, C. Wang, Integration of Carbon Nanotubes to C-MEMS for On-chip Supercapacitors. 2010, Nanotechnology, IEEE Transactions on, 9, 734-739.

[35] V. Penmatsa, J. H. Yang, Y. Yu, C. Wang, Fabrication of porous carbon micropillars using a block copolymer as porogen. 2010, Carbon, 48, 4109-4115.

[36] V. Penmatsa, T. Kim, M. Beidaghi, H. Kawarada, Z. Wang, L. Gu, C. Wang, 
Three-dimensional graphene nanosheet encrusted carbon micropillar arrays for electrochemical sensing. 2012, Nanoscale, DOI:10.1039/C2NR30161J.

[37] V. Penmatsa, H. Kawarada, C. Wang, Fabrication of carbon nanostructures using photo-nanoimprint lithography and pyrolysis. 2012, J. Micromech. Microeng, 22, 045024-045032.

[38] F. Tuinstra, J.L. Koenig, Raman Spectrum of Graphite. 1970, J. Chem. Phys., 53, 1126-1130.

[39] H. Kawarada, A. R. Ruslinda, Diamond electrolyte solution gate FETs for DNA and protein sensors using DNA/RNA aptamers. 2011, Phys. Status Solidi A, 208, 2005-2016.

[40] H. J. In, S. Kumar, Y. Shao-Horn, G. Barbastathis, Origami fabrication of nanostructured, three-dimensional devices: Electrochemical capacitors with carbon electrodes, 2006, Appl. Phys. Lett. 2006, 88, 083104 (pp.).

[41] Z. Weng, Y. Su, F. Li, J. Du, Graphene-Cellulose Paper Flexible Supercapacitors. 2011, Adv. Energy Mater. 2011, 1, 917-922.

[42] G. Wang, L. Zhang, J. Zhang, A review of electrode materials for electrochemical supercapacitors. 2012, Chem. Soc. Rev., 41, 797-828.

[43] P. L. Taberna, P. Simon, J. F. Fauvarque, Electrochemical Characteristics and Impedance Spectroscopy Studies of Carbon-Carbon Supercapacitors, 2003, J. Electrochem. Soc., 150, A292-A300.

[44] V. Presser, L. Zhang, J. J. Niu, J. McDonough, C. Perez, H. Fong, Y. Gogotsi, Flexible Nano-felts of Carbide-Derived Carbon with Ultra-high Power Handling Capability. 2011, Adv. Energy Mater., 1, 423-430.

[45] J. A. Paradiso, T. Starner, Energy Scavenging for Mobile and Wireless Electronics. 2005, IEEE Pervasive Comput., 4, 18-27. 


\section{CHAPTER 8}

\section{SUMMARY AND FUTURE WORK}

\subsection{Summary}

This dissertation presents fabrication, characterization, functionalization and validation of novel carbon micro and nano-structures for electrochemical and biosensing. The C-MEMS technique was used to fabricate 3D carbon microelectrodes arrays. It was previously demonstrated that these carbon structures can be used as functional units for electrochemical and biosensing applications. To further improve the surface area of the carbon microstructures for electrochemical sensing, two surface engineering strategies, i.e. using F127 as porogen and oxygen RIE treatment were employed to introduce surface porosity. A uniform mesoporous surface porosity was observed when F127 was used as porogen. Electrochemical BET results showed a $185 \%$ increase in the surface area of porous carbon electrode. Alternatively, in the case of oxygen plasma RIE treatment, hierarchical microporous morphology was evident on the surface. The flexibility to tailor the total surface area of the carbon microstructures makes it a promising process for future C-MEMS applications.

In an alternate approach, 3D C-MEMS arrays were explored as a high surface area platform for the integration of graphene. Graphene was conformally coated on the complex 3D structures using a spray deposition technique called electrostatic spray deposition (ESD). A thorough study of the effect of ESD processing conditions on the morphology of the deposited graphene film was investigated. Electrochemical analysis has shown that graphene/ carbon micropillar electrode array platform showed faster charge transfer and higher electrochemical activity towards $\mathrm{H}_{2} \mathrm{O}_{2}$ compared to bare 
carbon micropillar electrode array platform. This methodology promises a simple approach to coat various functional nanomaterials onto 3D microelectrode arrays with controllable morphology and employ them for electrochemical sensing.

For the first time the applicability of C-NEMS process to fabricate controllable nanostructures by photo-nanoimprint lithography and pyrolysis was demonstrated. The carbonized nanostructures exhibited microstructure comparative to the glassy carbon materials produced by pyrolyzing organic materials. Additionally, the electrical properties exhibited by carbon nanostructures were comparable to bulk glassy carbon. Finally, the carbon nanostructures were functionalized using direct amination technique for potential use as functional units in biological sensors. Controllable carbon features with nano/micro scale dimensions conceived by this cost-effective high throughput manufacturing technique will potentially enable us to explore their use in functional nanodevices.

To optimize the carboxyl functional group coverage on the carbon surface, oxidation of C-MEMS derived carbon films by four different oxidation techniques (VUV pretreatment, electrochemical activation, oxygen RIE pretreatment and $\mathrm{UV} / \mathrm{O}_{3}$ pretreatment) was investigated by XPS. All the oxidation techniques except $\mathrm{UV} / \mathrm{O}_{3}$ pretreatment yielded similar oxidation levels. Only $\mathrm{UV} / \mathrm{O}_{3}$ pretreatment yielded somewhat lower values which could be attributed to a comparatively low intensity of the UV-source. Compared to electrochemical activation and oxygen RIE pretreatments, the VUV and UV/O3 photochemical pretreatment techniques took longer time to reach a saturation limit. In all the oxidation techniques, by analysis of the $\mathrm{C} 1 \mathrm{~s}$ core level several coexisting oxygen-containing groups were detected with the carboxyl group increasing 
with pretreatment time. Finally this study gives us the ability to choose an oxidation technique for the optimization of functional groups conducive to the covalent binding of bioreceptors.

In the end, this work demonstrates the high sensitive detection of one of the cancer biomarkers called PDGF-BB using aptamer/protein binding complex on functionalized 3D carbon microarray platform. For covalent immobilization of the probe aptamer, the carbon surface was bio-functionalized by direct amination technique. The sensor showed a near linear relationship towards protein concentration even in the subnanomolar range $(5 \mathrm{pmol})$ with excellent selectivity against similar interferences. The robust platform of signaling aptamer/protein binding complex on 3D carbon microarrays has the ability to detect wide variety of biomarkers and proteins for potential application in the preliminary diagnosis of cancer.

\subsection{Future Scope of this Work}

This dissertation has introduced various ideas to fabricate high surface area carbon micro/nanostructures along with hybrid carbon materials integrating functional nanomaterials onto high surface area microstructure platforms. The proposed strategies suggest new possibilities for development of high sensitive electrochemical and biosensing devices.

In the present dissertation, the application of C-MEMS structures as high surface area platform was demonstrated by conformally coating graphene onto 3D micropillar arrays. Going further, different functional nanomaterials can be integrated on the 3D carbon microarrays and these hybrid carbon structures have huge potential in chemical and biological sensors that needs to be explored. Because of the controllability of the 
functionalized carbon nanostructures fabricated in this work, they can be seamlessly integrated into future miniaturized biosensors. One of the major achievements of this dissertation was the high sensitive detection of platelet-derived growth factor, a potential cancer biomarker using functionalized 3D carbon microarrays platform. This platform can further be explored for the detection of wide variety of biomarkers and proteins for potential application in the preliminary diagnosis of cancer. 
VITA

\section{VARUN PENMATSA}

2003-2007

2007-2009

2009-2012
B.Tech., Electronics and Communications Engineering Jawaharlal Nehru Technological University

Hyderabad, India (GPA-3.52/4.00)

M.S., Material Science and Engineering

Florida International University

Miami, Fl, USA (GPA-3.53/4.00)

Doctoral Candidate $(\mathrm{PhD})$, Material Science and Engg.

Florida International University

Miami, Fl, USA (GPA-3.82/4.00)

\section{AWARDS AND HONORS}

1. Dissertation Year Fellowship (January 2012- December 2012) awarded by Florida International University

2. Dissertation Evidence Acquisition Fellowship (May 2011-December 2011) awarded by Florida International University

3. First Prize in Art inside Materials competition conducted by ASM-TMS chapter at FIU, 2012

4. Finalist in "Science as Art" competition held at MRS Spring 2008 Symposium. 50 images were chosen to compete from 200 high quality images sent from across the world.

5. Scholarship from OAI (Optical Associated Inc.) company to present research work at MRS Spring 2011 Meeting \& Exhibit

\section{PUBLICATIONS/ BOOK CHAPTER}

1. Varun Penmatsa, Ruslinda .A. Rahim, Majid Beidaghi, Hiroshi Kawarada, Chunlei Wang "Detection of Platelet-derived growth factor (PDGF) on Functionalized 3D Microarrays Platform", Biosensors and Bioelectronics (2013), 39, 118-123.

2. Varun Penmatsa, Taekwon Kim, Majid Beidaghi, Hiroshi Kawarada, Zhifeng Wang, Lin Gu, Chunlei Wang “Three-Dimensional Graphene Nanosheets Encrusted Carbon Micropillar Arrays for Electrochemical Sensing", Nanoscale (2012), DOI: 10.1039/C2NR30161J. This work was highlighted as the cover page of "Nanoscale" 
journal and in Spotlight section of "Nanowerk" http://www.nanowerk.com/spotlight/spotid=24995.php

3. Varun Penmatsa, Hiroshi Kawarada, Chunlei Wang "Fabrication of Carbon Nanostructures using Photo-Nanoimprint Lithography and Pyrolysis", J. Micromech. Microeng. (2012), 22, 045024-045031.

4. Varun Penmatsa, Jung-Hoon Yang, Yan Yu, Chunlei Wang "Fabrication of Porous 3-Dimensional Carbon Micropillars using Block Copolymer as Porogen", Carbon (2010), volume 48 (14), 4109-4115.

5. Varun Penmatsa, Ruslinda .A. Rahim, Majid Beidaghi, Hiroshi Kawarada, Chunlei Wang "Functionalized Three-Dimensional Carbon Microarrays for Cancer Biomarker Detection", ECS transactions (submitted).

6. Varun Penmatsa, Ruslinda A. Ruslinda, Hiroshi Kawarada, Chunlei Wang "Characterization of Vacuum-Ultraviolet (VUV) Functionalization of Pyrolyzed Carbon and its Application for HIV-TAT Protein Detection", Langmuir (in preparation).

7. Varun Penmatsa, Ruslinda A. Ruslinda, Majid Beidaghi, Hiroshi Kawarada, Chunlei Wang "Comparison of Different Surface Functionalization Techniques on Pyrolyzed Photoresist Carbon", (in preparation).

8. Yin Song, Varun Penmatsa, Chunlei Wang "Recent Development of Miniature Enzymatic Biofuel Cells" (2011), Intech publishers (Book chapter).

9. Ruslinda Rahim, Varun Penmatsa, Yoko Ishii, Shinya Tajima, Hiroshi Kawarada "High Sensitive Detection of Platelet-Derived Growth Factor on a Functionalized Diamond Surface using Aptamer Sandwich Design”, Analyst (2012), 137, 1692-1697.

10. Yin Song, Varun Penmatsa, Chunlei Wang "Recent Development of Miniature Enzymatic Biofuel Cell", Proceedings of the SPIE (2011), 8035, DOI: $10.1117 / 12.885569$.

11. Wei Chen, Majid Beidaghi, Varun Penmatsa, Latha Kumari, Wenzhi Li, and Chunlei Wang , "Integration of Carbon Nanotubes to C-MEMS for On-chip Supercapacitors" , IEEE Transactions On Nanotechnology (2010), 9, 734-740. 